\title{
Dual Photoredox/Nickel-Promoted Alkylation of Heteroaryl Halides with Redox-Active Esters
}

Nicole Erin Behnke, ${ }^{1}$ Zachary S. Sales, ${ }^{2}$ Minyan Li, ${ }^{2}$ Aaron T. Herrmann ${ }^{2 *}$

${ }^{1}$ Department of Chemistry, Rice University, BioScience Research Collaborative, 6500 Main Street, Rm 380, Houston, TX 77030 (USA), ${ }^{2}$ Janssen Research \& Development L.L.C., Discovery Process Research, 3210 Merryfield Row, San Diego, CA 92121 (USA)

Corresponding author E-mail: aherrman@its.jnj.com

Supporting Information

Table of Contents

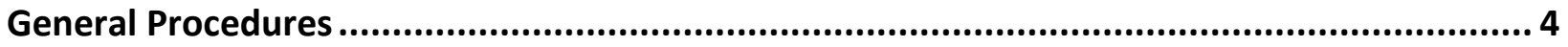

Procedure for Alkylation of Halogenated Pyrimidines with Redox-Active Esters...............................4

Scheme S1. Photochemical/Ni-Promoted Alkylation of Heteroaryl Pyrimidines with Redox-Active

Esters.

Figure S1. Guide for Ir/Ni-Promoted Cross-Coupling of Pyrimidine 1 with Redox-Active Ester 2 ........5

Reaction Optimization and Scope Evaluation using High Throughput Experimentation Conducted at the Discovery Process Research HTE Lab.

Table S1. High-Throughput Experimentation Results for Photochemical/Ni-Promoted Cross-

Coupling of Pyrimidine 1 and Redox-Active Ester 36.

Scheme S2. High-Throughput Experimentation for Photochemical/Ni-Promoted Cross-Coupling of

Heteroaryl Halides and Redox-Active Esters.

Table S2. Results of High-Throughput Experimentation for Product 3. .....................................10

Table S3. Results of High-Throughput Experimentation for Product 6. ........................................11

Table S4. Results of High-Throughput Experimentation for Product 7. ......................................12

Table S5. Results of High-Throughput Experimentation for Product 8. .......................................13

Table S6. Results of High-Throughput Experimentation for Product 12. ....................................14

Table S7. Results of High-Throughput Experimentation for Product 14. ........................................15 
Table S8. Results of High-Throughput Experimentation for Product 16.

Optimization of Reaction Conditions for Alkylation of Halogenated Pyrimidines with Redox-

Active Esters.

Table S9: Optimization of Reaction Conditions for Alkylation of Pyrimidine 1 with Cyclopentane

Redox-Active Ester 2

Table S10: Optimization of Reaction Conditions for Alkylation of Pyrimidine 1 with Cyclobutane

Redox-Active Ester 46

NMR Characterization 19

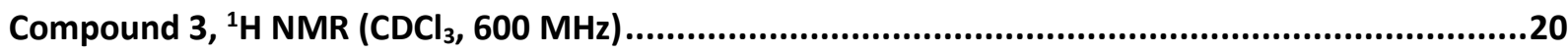

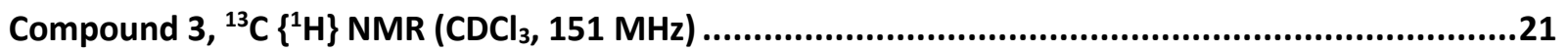

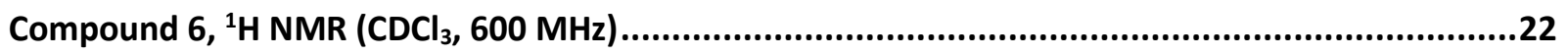

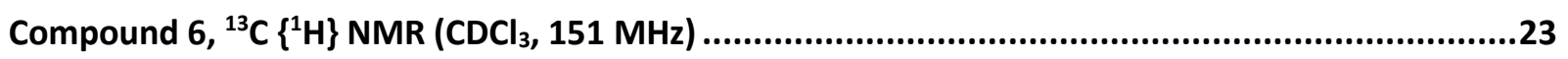

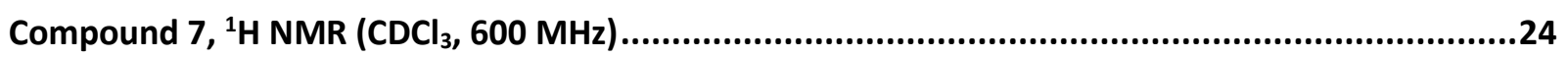

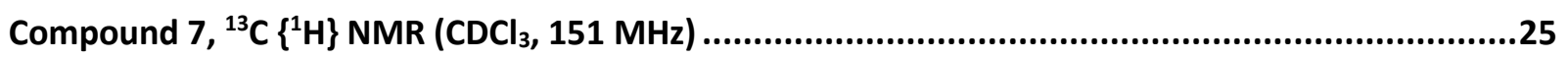

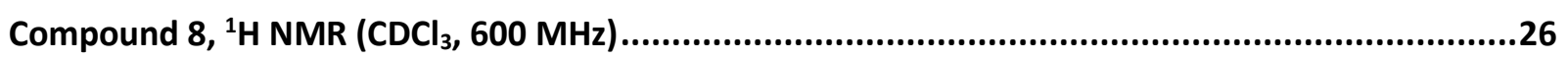

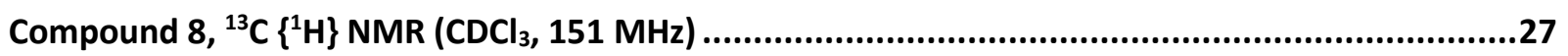

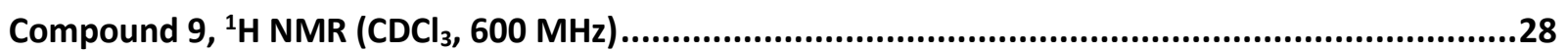

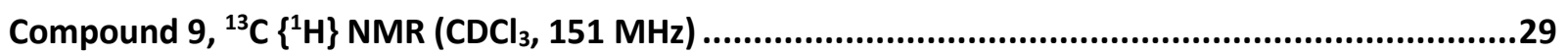

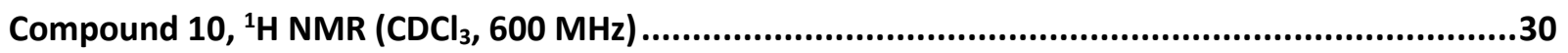

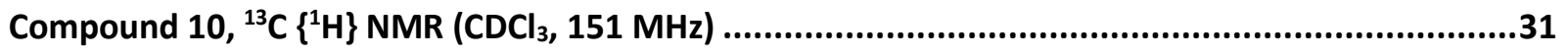

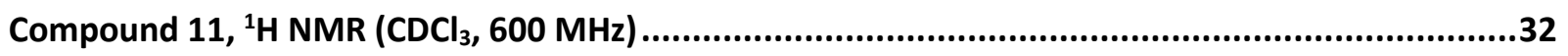

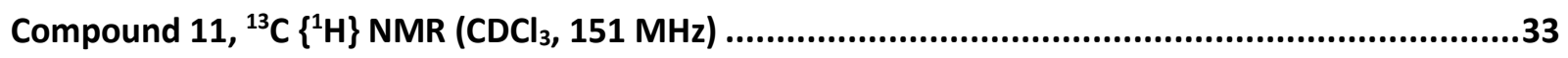

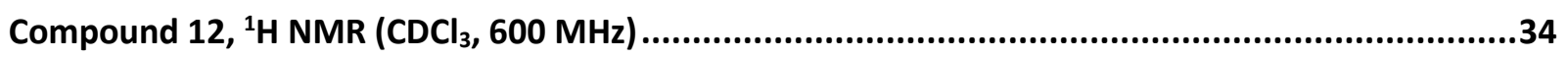

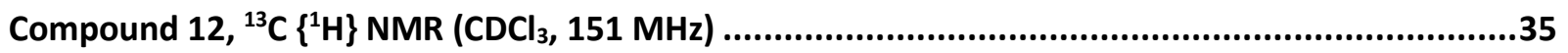

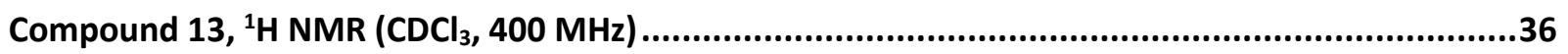

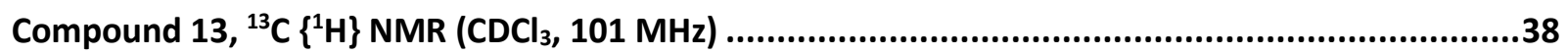

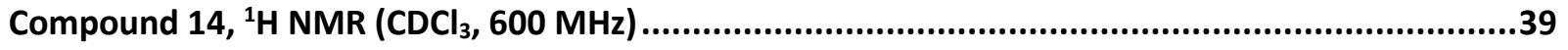

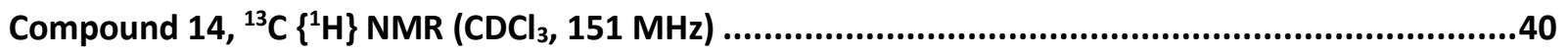

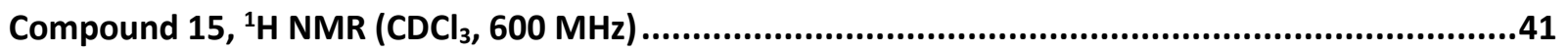

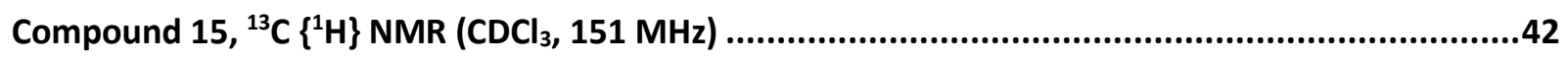

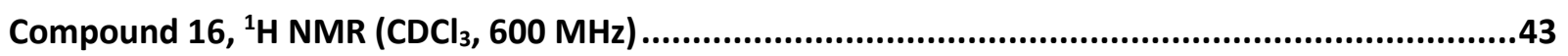

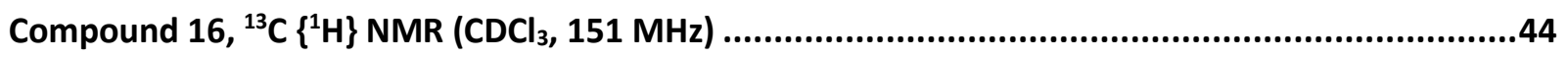

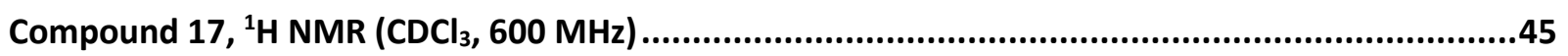

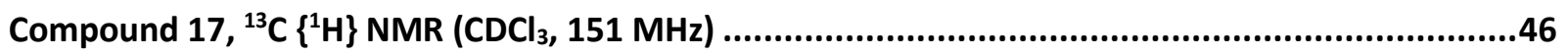

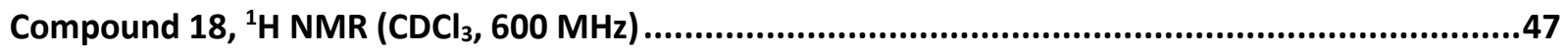




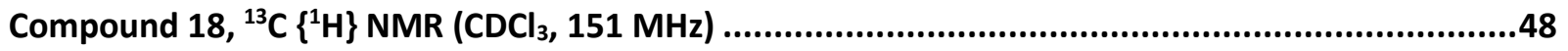

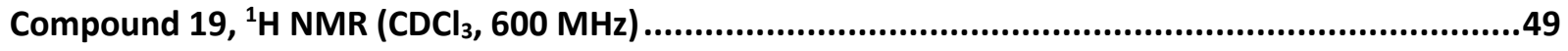

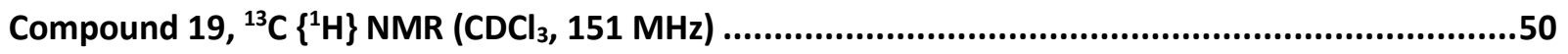

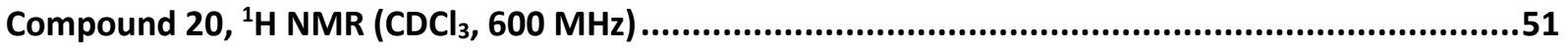

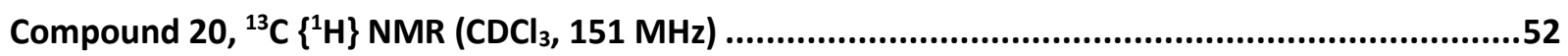

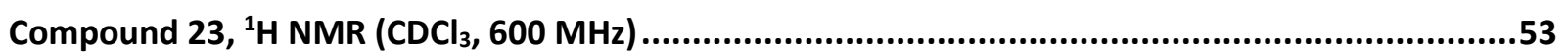

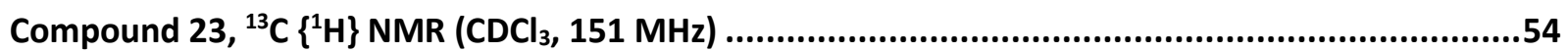

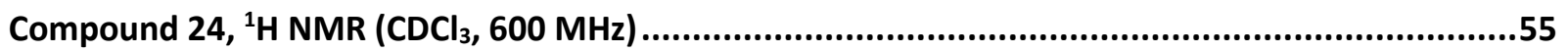

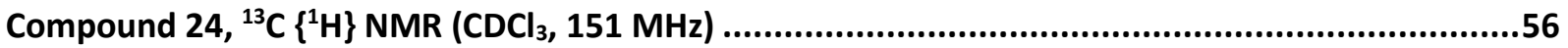

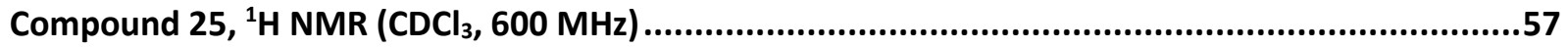

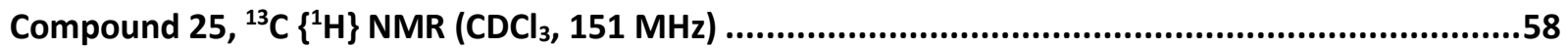

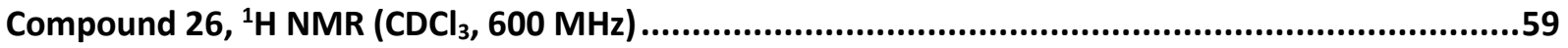

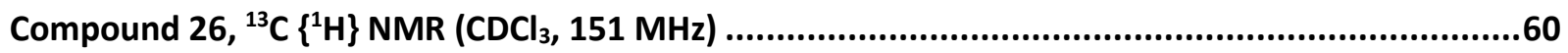

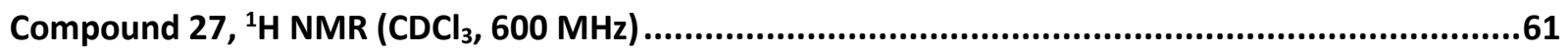

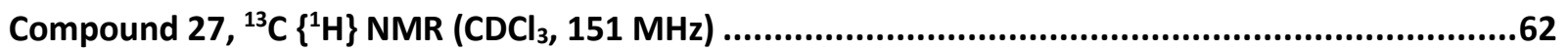

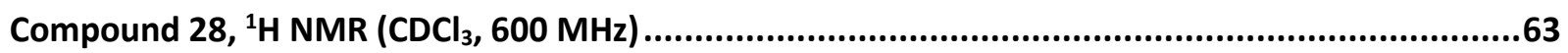

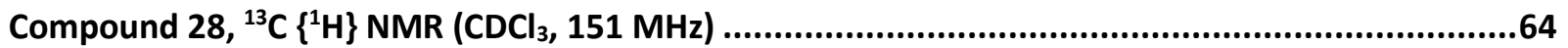

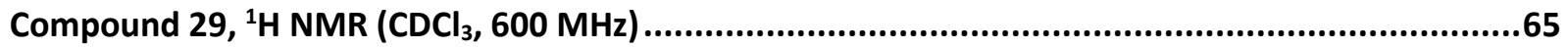

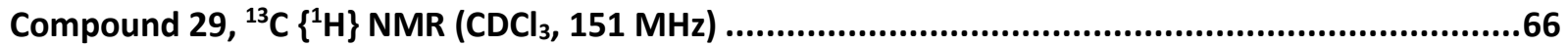

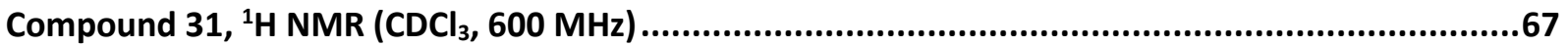

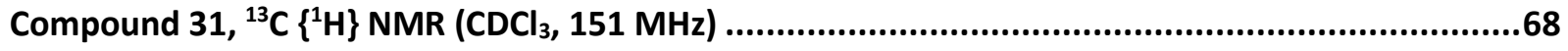

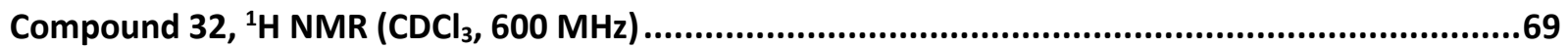

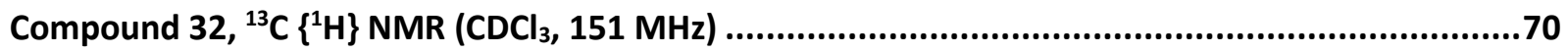

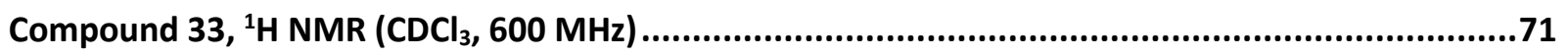

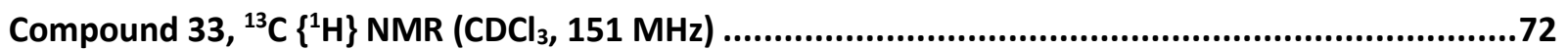

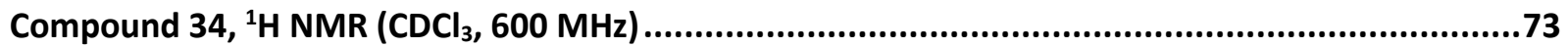

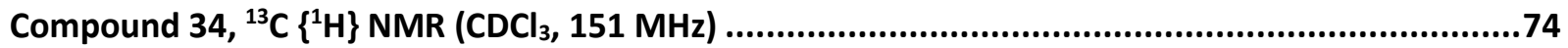

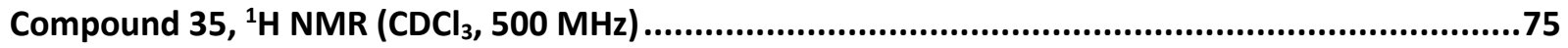

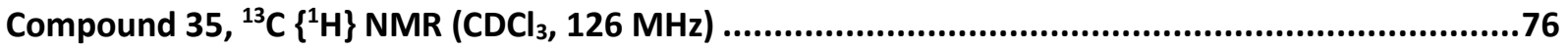




\section{General Procedures}

Procedure for Alkylation of Halogenated Pyrimidines with Redox-Active Esters

Scheme S1. Photochemical/Ni-Promoted Alkylation of Heteroaryl Pyrimidines with Redox-Active Esters.

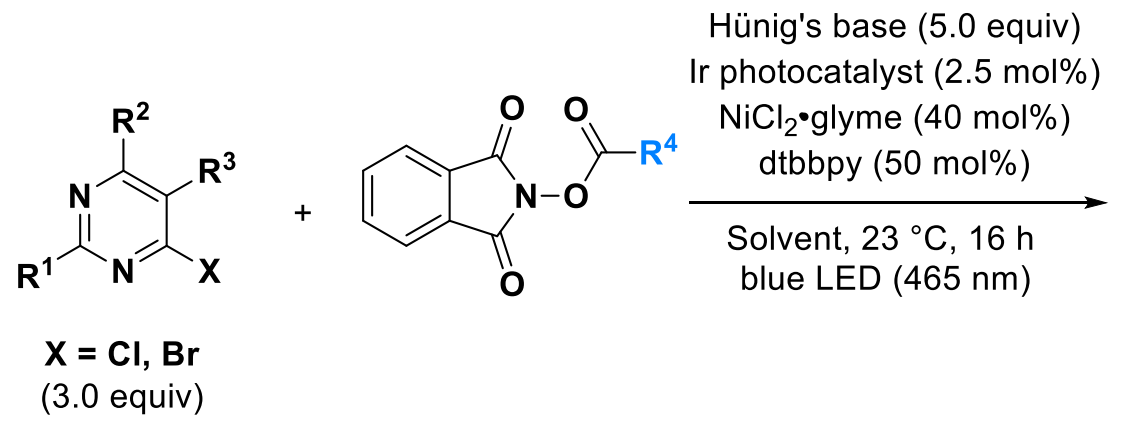

Vial 1': To a 1 dram vial purged and backfilled with $\mathrm{N}_{2}(3 X)$ and equipped with a magnetic stir bar, 4,4'-di-tert-butyl-2,2'-bipyridine $(0.1 \mathrm{mmol}, 50 \mathrm{~mol} \%)$ and Nickel(II) chloride ethylene glycol dimethyl ether complex $(0.08 \mathrm{mmol}, 40 \mathrm{~mol} \%)$ were dissolved in the corresponding solvent (1.33 $\mathrm{mL}, 0.15 \mathrm{M})$. The vial was sealed and heated to $30^{\circ} \mathrm{C}$ with vigorous stirring for 15 minutes. The solution was removed from heat and stirred for an additional 15 minutes at $23^{\circ} \mathrm{C}$.

Vial 2: To a 1 dram vial equipped with a magnetic stir bar was added pyrimidine $(0.6 \mathrm{mmol}, 3.0$ equiv), redox-active ester ( $0.2 \mathrm{mmol}, 1.0$ equiv), and the corresponding Ir photocatalyst (0.005 mmol, 2.5 mol\%). The vial was evacuated and backfilled with $\mathrm{N}_{2}(3 \mathrm{X})$. The solution from Vial 1 was added to Vial 2. Hünig's base ( $1.0 \mathrm{mmol}, 5.0$ equiv) was added and the reaction was exposed to blue LED ( $465 \mathrm{~nm}$ ) while stirring with overhead fan for 16 hours. After the reaction time, the solution was poured into $\mathrm{H}_{2} \mathrm{O}(15 \mathrm{~mL})$ and extracted with EtOAc $(3 \times 15 \mathrm{~mL})$. The combined organic layers were washed with one portion of brine $(25 \mathrm{~mL})$ and dried over anhydrous $\mathrm{Na}_{2} \mathrm{SO}_{4}$. The crude material was concentrated in vacuo and purified by column chromatography to provide the desired product. 
Figure S1. Guide for Ir/Ni-Promoted Cross-Coupling of Pyrimidine 1 with Redox-Active Ester 2
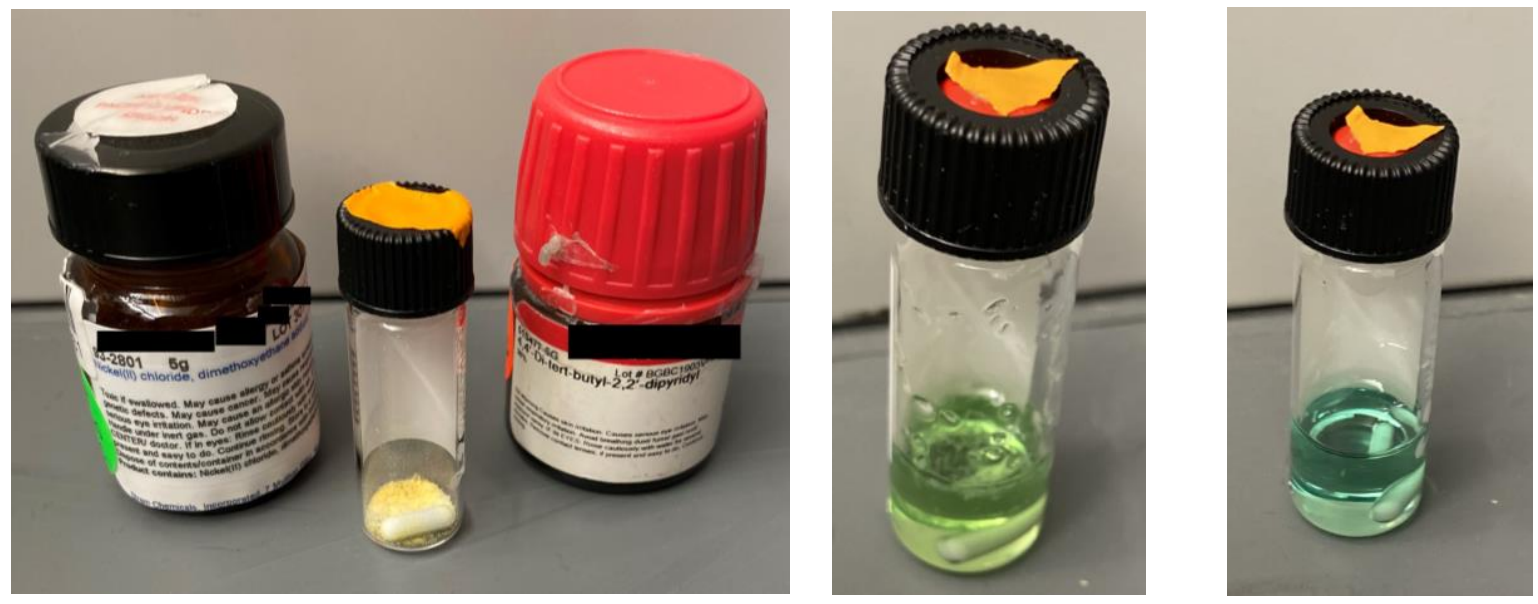

Left: Reagents in Vial 1. Center: Vial 1 dissolved in DMSO. Right: Vial 1 after 30 minute stirring.
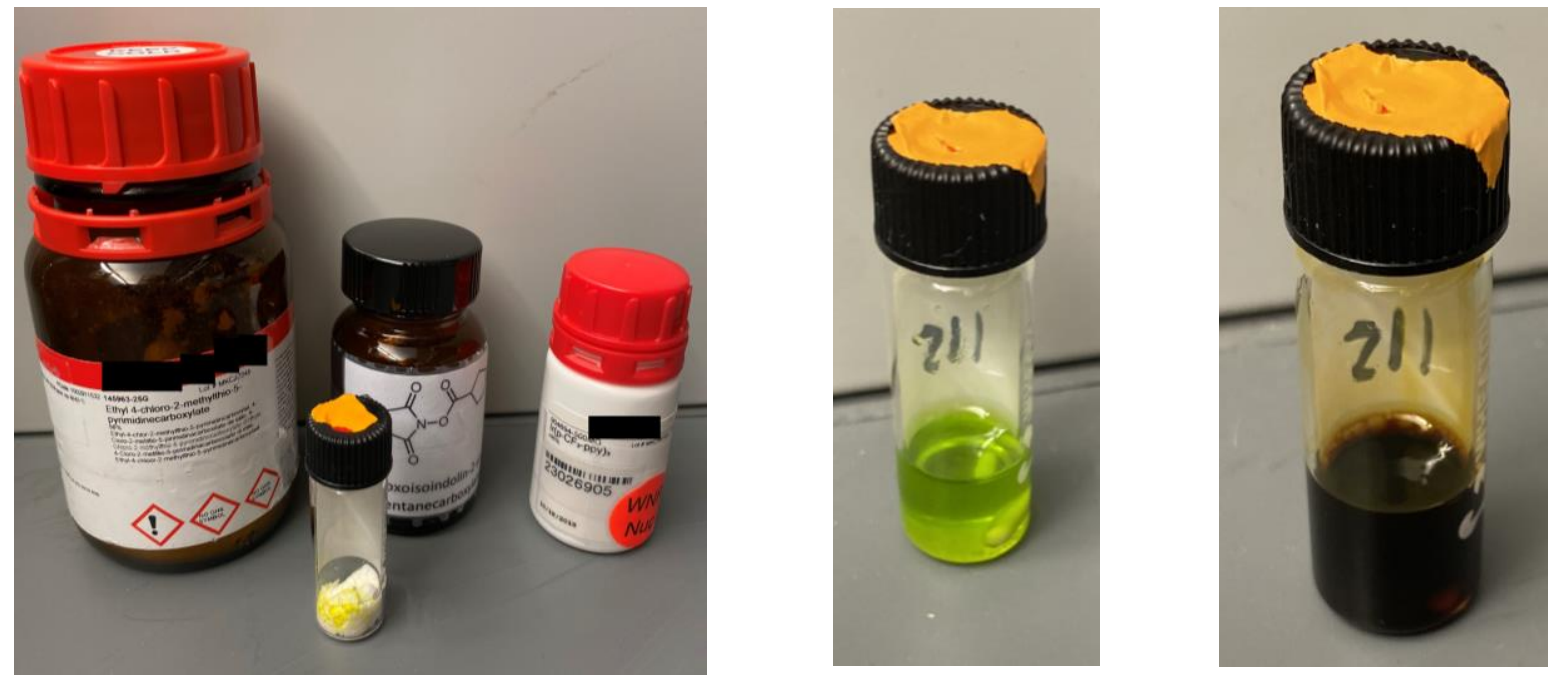

Left: Reagents in Vial 2. Center: After Vial 1 is combined with Vial 2. Right: After overnight exposure to blue LED. 


\section{Reaction Optimization and Scope Evaluation using High Throughput}

\section{Experimentation Conducted at the Discovery Process Research HTE Lab}

General Information. High-throughput Experimentation Screening was accomplished in a Vacuum Atmospheres glovebox with oxygen typically $<5 \mathrm{ppm}$. The experimental design was accomplished using Library Studio (Unchained Labs). Screening reactions were carried out in 1 $\mathrm{mL}$ vials (30 mm height $\times 8 \mathrm{~mm}$ diameter) in "Para-dox" standard 96-position photoredox aluminum reaction blocks (Analytical-sales). Liquid chemicals were dosed using multi-channel or single-channel pipettors. Solid chemicals were dosed manually as solutions or slurries in appropriate solvents. Undesired additional solvent was removed using a Speedvac system located inside the glovebox. The reactions were stirred at $23^{\circ} \mathrm{C}$ over Lumidox $96-$ well Blue LED array (analytical-sales) on an aluminum block with a tumble-stirrer (V\&P Scientific) using 1.98 $\mathrm{mm}$ diameter $\times 4.80 \mathrm{~mm}$ length parylene stir bars. The tumble stirring mechanism helped to insure uniform stirring throughout the 96 -well plate. Results of the screening were analyzed by Waters UPLC with addition of an internal standard. The UPLC areas of product over internal standard are shown in the tables below. Note that area ratios are pertinent only to the corresponding specific screening and are not quantitatively compared with different screening.

\section{Screening of Ir Photocatalyst.}

Procedure of High-Throughput Experimentation: In the glovebox, to a "Para-dox" standard 96position photoredox aluminum reaction block containing $1 \mathrm{~mL}$ reaction vials pre-plated with photocatalysts ( 0.05 equiv) and equipped with Teflon-coated magnetic stir bar was added sequentially: solution of pre-mixed $\mathrm{NiCl}_{2}$.glyme ( 0.2 equiv) and dtbbpy ( 0.2 equiv) dissolved in DMSO and solution of pyrimidine (3.0 equiv), redox-active ester (1.0 equiv), Hünig's base(5.0 equiv). The plate was sealed, moved out of glovebox, and was stirred over blue LED lights at $23^{\circ} \mathrm{C}$. After 16 hours, the reactions were opened to air and diluted with $500 \mu \mathrm{L}$ of a MeCN solution of 4,4'-Di-tert-butylbiphenyl. After stirring for $15 \mathrm{~min}$, the vials were centrifuged with Genevac and $50 \mu \mathrm{L}$ aliquots were taken from the reaction vials and dosed into a 96 well UPLC collection plate. These aliquots were further diluted by addition of $600 \mu \mathrm{L}$ of MeCN. The reactions were analyzed by UPLC. Area ratio of product over internal standard was further converted to assay yield using calibration curve generated from product standard. 
Table S1. High-Throughput Experimentation Results for Photochemical/Ni-Promoted Cross-Coupling of Pyrimidine 1 and Redox-Active Ester 36.

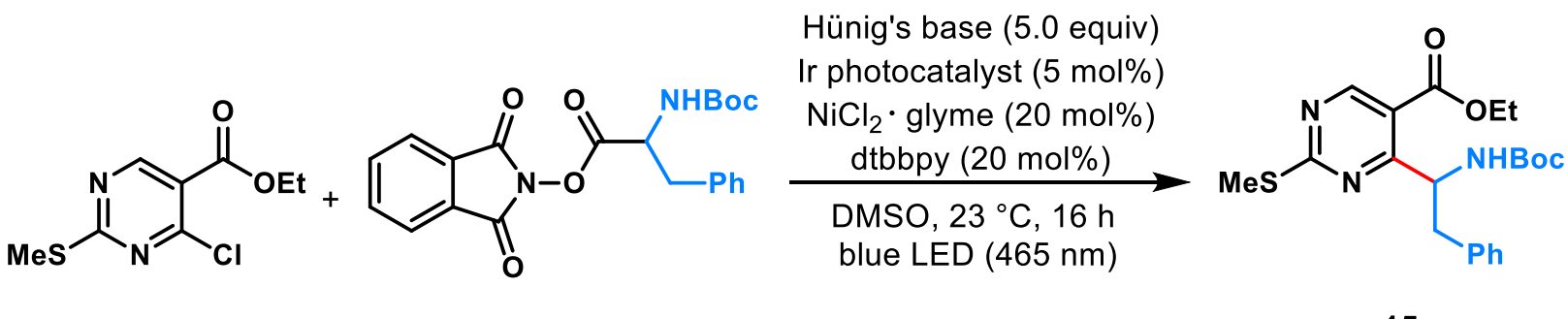

(3.0 equiv)

\begin{tabular}{|c|c|c|}
\hline Photocatalyst & $\begin{array}{l}\text { Pdt/IS Area } \\
\text { Ratio }\end{array}$ & Assay Yield \\
\hline $\begin{array}{l}\text { [4,4'-Bis(1,1-dimethylethyl)-2,2'-bipyridine]bis[3,5-difluoro-2-(5-methyl-2- } \\
\text { pyridinyl)phenyl] iridium }\end{array}$ & 0.39 & 39.44 \\
\hline $\begin{array}{l}\text { [4,4'-Bis(1,1-dimethylethyl)-2,2'-bipyridine]bis[3,5-difluoro-2-(2-pyridinyl- } \\
\text { KN)phenyl-kC]iridium }\end{array}$ & 0.33 & 32.74 \\
\hline $\begin{array}{l}\text { [4,4'-Bis(1,1-dimethylethyl)-2,2'-bipyridine]bis[3,5-difluoro-2-(5-fluoro-2- } \\
\text { pyridinyl)phenyl]iridium }\end{array}$ & 0.33 & 32.78 \\
\hline Tris[(2-(2-pyridinyl-kN)-5-(trifluoromethyl)phenyl-kC]iridium(III) & 0.48 & 47.83 \\
\hline $\begin{array}{l}\text { [4,4'-Bis(1,1-dimethylethyl)-2,2'-bipyridine]bis[5-fluoro-2-(5-methyl-2- } \\
\text { pyridinyl)phenyl-]iridium }\end{array}$ & 0.41 & 40.99 \\
\hline $\begin{array}{l}\text { (2,2'-Bipyridine)bis[3,5-difluoro-2-[5-(trifluoromethyl)-2-pyridinyl][phenyl- } \\
\text { kC]iridium(III) }\end{array}$ & 0.44 & 43.97 \\
\hline 10-Phenylphenothiazine & 0.18 & 17.62 \\
\hline $\begin{array}{l}\text { 4,4'-Bis(t-butyl-2,2'-bipyridine]bis[5-methyl-2-(4-methyl-2-pyridinyl-kN)phenyl- } \\
\text { kC]iridium }\end{array}$ & 0.44 & 44.48 \\
\hline [Ir(dtbbpy)(ppy)2]PF6 & 0.40 & 40.05 \\
\hline $\begin{array}{l}\text { (4,4'-Di-t-butyl-2,2'-bipyridine)bis[3,5-difluoro-2-[5-trifluoromethyl-2-pyridinyl- } \\
\text { kN)phenyl-kC]iridium(III) }\end{array}$ & 0.38 & 38.06 \\
\hline 9-Mesityl-3,6-di-tert-butyl-10-phenylacridinium & 0.00 & 0.00 \\
\hline Eosin Y & 0.27 & 27.21 \\
\hline [Ru(phen)3]Cl2 & 0.00 & 0.00 \\
\hline [Ru(dmbpy)3](PF6)2 & 0.26 & 25.55 \\
\hline Ru(p-CF3-bpy)3 (BF4)2 & 0.36 & 36.22 \\
\hline Tris[4,4'-bis(t-butyl)-2,2'-bipyridine]ruthenium(II) hexafluorophosphate & 0.30 & 30.24 \\
\hline$[R u(b p z) 3][P F 6] 2$ & 0.00 & 0.00 \\
\hline$[\mathrm{Ru}(\mathrm{bpm}) 3][\mathrm{Cl}] 2$ & 0.02 & 1.84 \\
\hline Ru(BPY)3 & 0.31 & 31.26 \\
\hline Ru(bpy)3(PF6)2 & 0.38 & 38.04 \\
\hline Tris(1,10-phenanthroline)ruthenium(II) chloride hydrate & 0.21 & 20.46 \\
\hline 9-Mesityl-2,7-dimethyl-10-phenylacridinium tetrafluoroborate & 0.00 & 0.00 \\
\hline 10-Methyl-9-(2,4,6-trimethylphenyl)acridinium perchlorate & 0.00 & 0.00 \\
\hline 4,4'-Bis(N-carbazolyl)-1,1'-biphenyl & 0.26 & 25.57 \\
\hline
\end{tabular}




\section{Substrate Evaluation.}

Procedure of High-Throughput Experimentation: To a "Para-dox" standard 96-position photoredox aluminum reaction block containing $1 \mathrm{~mL}$ reaction vials equipped with Teflon-coated magnetic stir bar was added solution of photocatalyst ( 0.05 equiv) in THF or $\mathrm{MeOH}$, the solvents were then removed via Genevac. To certain vials (shown in Tables below), Tetrabutylammonium iodide ( 1 equiv) was added as a slurry in THF, which were then removed via Genevac. In the glovebox, to the 96 well plate was added sequentially, (1) solution of pre-mixed $\mathrm{NiCl}_{2} \cdot$ glyme $(0.2$ equiv) and dtbbpy (0.2 equiv) dissolved in DMSO or Dioxane, (2) solution of pyrimidine (3.0 equiv) dissolved in DMSO or Dioxane, (3) solution of redox-active ester (1.0 equiv), Hünig's base (5.0 equiv) dissolved in DMSO or Dioxane. The plate was sealed, moved out of glovebox, and was stirred over blue LED lights at $23^{\circ} \mathrm{C}$. After 16 hours, the reactions were opened to air and diluted with $500 \mu \mathrm{L}$ of a MeCN solution of 4,4'-Di-tert-butylbiphenyl. After stirring for $15 \mathrm{~min}$, the vials were centrifuged with Genevac and $50 \mu \mathrm{L}$ aliquots were taken from the reaction vials and dosed into a 96 well UPLC collection plate. These aliquots were further diluted by addition of $600 \mu \mathrm{L}$ of $\mathrm{MeCN}$. The reactions were analyzed by UPLC. Area ratio of product over internal standard were used to evaluate the reaction conditions for each substrate. 
Scheme S2. High-Throughput Experimentation for Photochemical/Ni-Promoted CrossCoupling of Heteroaryl Halides and Redox-Active Esters.

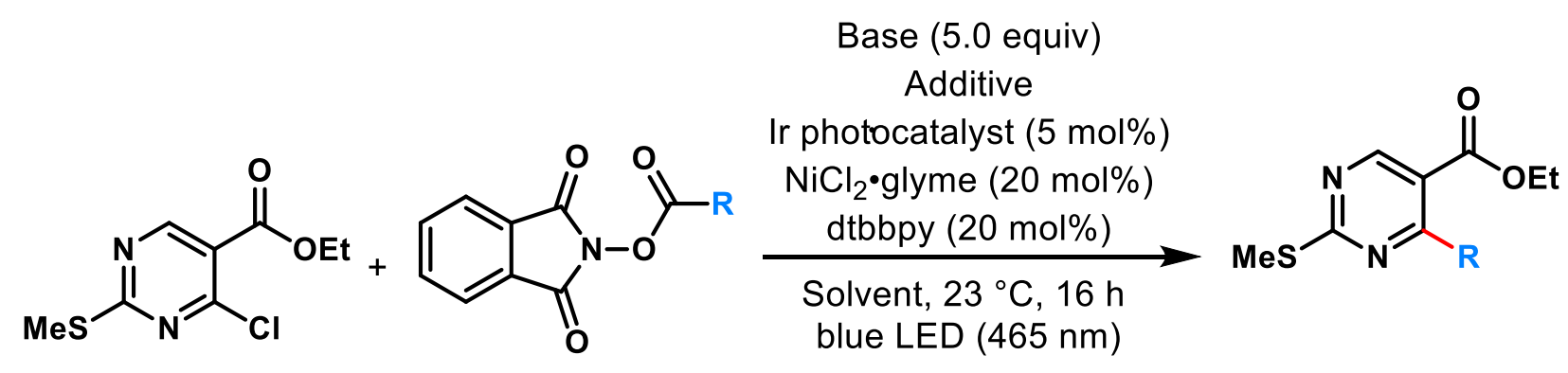

(3.0 equiv)

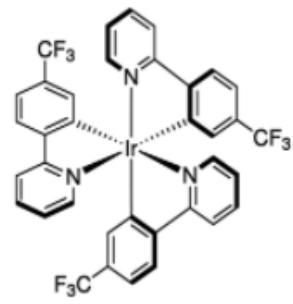

PC1

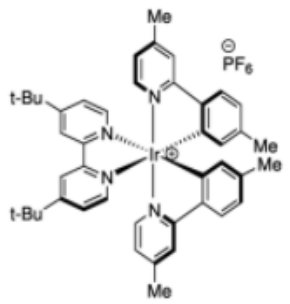

PC2

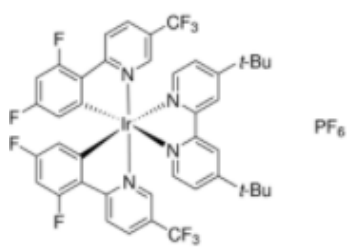

PC3 
Table S2. Results of High-Throughput Experimentation for Product 3.<smiles>CCOC(=O)c1cnc(S(C)(=O)=O)nc1C1CCCC1</smiles>

3

\begin{tabular}{|l|l|l|l|}
\hline Solvent & Photocatalyst CAS\# & Base & Ratio \\
\hline DMSO & PC1 500295-52-3 & DIPEA & 0.60 \\
\hline DMSO & PC2 1607469-49-7 & DIPEA & $\mathbf{0 . 5 2}$ \\
\hline DMSO & PC3 870987-63-6 & DIPEA & $\mathbf{0 . 3 4}$ \\
\hline DMSO & PC1 500295-52-3 & DIPEA+TBAI & $\mathbf{0 . 4 1}$ \\
\hline DMSO & PC2 1607469-49-7 & DIPEA+TBAI & $\mathbf{0 . 4 2}$ \\
\hline DMSO & PC3 870987-63-6 & DIPEA+TBAI & $\mathbf{0 . 2 7}$ \\
\hline Dioxane & PC1 500295-52-3 & DIPEA & $\mathbf{0 . 2 5}$ \\
\hline Dioxane & PC2 1607469-49-7 & DIPEA & $\mathbf{0 . 4 5}$ \\
\hline Dioxane & PC3 870987-63-6 & DIPEA & $\mathbf{0 . 0 5}$ \\
\hline Dioxane & PC1 500295-52-3 & DIPEA+TBAI & $\mathbf{0 . 1 8}$ \\
\hline Dioxane & PC2 1607469-49-7 & DIPEA+TBAI & $\mathbf{0 . 1 4}$ \\
\hline Dioxane & PC3 870987-63-6 & DIPEA+TBAI & $\mathbf{0 . 0 0}$ \\
\hline
\end{tabular}


Table S3. Results of High-Throughput Experimentation for Product 6.

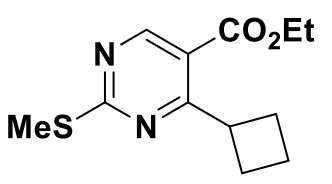

6

\begin{tabular}{|l|l|l|}
\hline Solvent & Photocatalyst CAS\# & Ratio \\
\hline DMSO & PC1 500295-52-3 & 0.29 \\
\hline DMSO & PC2 1607469-49-7 & 0.24 \\
\hline DMSO & PC3 870987-63-6 & 0.17 \\
\hline DMSO & PC4 676525-77-2 & 0.27 \\
\hline DMSO & PC5 60804-74-2 & 0.00 \\
\hline DMSO & PC 6 808142-88-3 & 0.21 \\
\hline Dioxane & PC1 500295-52-3 & 0.35 \\
\hline Dioxane & PC2 1607469-49-7 & 0.40 \\
\hline Dioxane & PC3 870987-63-6 & 0.64 \\
\hline Dioxane & PC4 676525-77-2 & 0.78 \\
\hline Dioxane & PC5 60804-74-2 & 0.00 \\
\hline Dioxane & PC 6 808142-88-3 & 0.53 \\
\hline
\end{tabular}


Table S4. Results of High-Throughput Experimentation for Product 7.<smiles>CCOC(=O)c1cnc(SC)nc1C1CC1</smiles>

7

\begin{tabular}{|l|l|l|}
\hline Solvent & Photocatalyst CAS\# & Ratio \\
\hline DMSO & PC1 500295-52-3 & 0.59 \\
\hline DMSO & PC2 1607469-49-7 & 0.61 \\
\hline DMSO & PC3 870987-63-6 & 0.56 \\
\hline DMSO & PC4 676525-77-2 & 0.64 \\
\hline DMSO & PC5 60804-74-2 & 0.40 \\
\hline DMSO & PC 6 808142-88-3 & 0.58 \\
\hline Dioxane & PC1 500295-52-3 & 0.07 \\
\hline Dioxane & PC2 1607469-49-7 & 0.05 \\
\hline Dioxane & PC3 870987-63-6 & 0.05 \\
\hline Dioxane & PC4 676525-77-2 & 0.05 \\
\hline Dioxane & PC5 60804-74-2 & 0.17 \\
\hline Dioxane & PC 6 808142-88-3 & 0.06 \\
\hline
\end{tabular}


Table S5. Results of High-Throughput Experimentation for Product 8.<smiles>CCOC(=O)c1cnc(SC)nc1C(C)C</smiles>

8

\begin{tabular}{|l|l|l|l|}
\hline Solvent & Photocatalyst CAS\# & Base & Ratio \\
\hline DMSO & PC1 500295-52-3 & DIPEA & 0.43 \\
\hline DMSO & PC2 1607469-49-7 & DIPEA & 0.30 \\
\hline DMSO & PC3 870987-63-6 & DIPEA & 0.22 \\
\hline DMSO & PC1 500295-52-3 & DIPEA+TBAI & 0.37 \\
\hline DMSO & PC2 1607469-49-7 & DIPEA+TBAI & 0.25 \\
\hline DMSO & PC3 870987-63-6 & DIPEA+TBAI & 0.18 \\
\hline Dioxane & PC1 500295-52-3 & DIPEA & 0.33 \\
\hline Dioxane & PC2 1607469-49-7 & DIPEA & 0.74 \\
\hline Dioxane & PC3 870987-63-6 & DIPEA & 0.07 \\
\hline Dioxane & PC1 500295-52-3 & DIPEA+TBAI & 0.29 \\
\hline Dioxane & PC2 1607469-49-7 & DIPEA+TBAI & 0.31 \\
\hline Dioxane & PC3 870987-63-6 & DIPEA+TBAI & 0.02 \\
\hline
\end{tabular}


Table S6. Results of High-Throughput Experimentation for Product 12.<smiles>CCOC(=O)c1cnc(S(C)(=O)=O)nc1C1CCNCC1</smiles>

12

\begin{tabular}{|l|l|l|}
\hline Solvent & Photocatalyst CAS\# & Ratio \\
\hline DMSO & PC1 500295-52-3 & 0.33 \\
\hline DMSO & PC2 1607469-49-7 & 0.26 \\
\hline DMSO & PC3 870987-63-6 & 0.24 \\
\hline DMSO & PC4 676525-77-2 & 0.25 \\
\hline DMSO & PC5 60804-74-2 & 0.00 \\
\hline DMSO & PC 6 808142-88-3 & 0.25 \\
\hline Dioxane & PC1 500295-52-3 & 0.39 \\
\hline Dioxane & PC2 1607469-49-7 & 0.70 \\
\hline Dioxane & PC3 870987-63-6 & 0.87 \\
\hline Dioxane & PC4 676525-77-2 & 0.61 \\
\hline Dioxane & PC5 60804-74-2 & 0.00 \\
\hline Dioxane & PC 6 808142-88-3 & 0.67 \\
\hline
\end{tabular}


Table S7. Results of High-Throughput Experimentation for Product 14.<smiles>CCOC(=O)c1cnc(S(C)(=O)=O)nc1C(CC(C)C)NC(=O)OCc1ccccc1</smiles>

14

\begin{tabular}{|l|l|l|}
\hline Solvent & Photocatalyst CAS\# & Ratio \\
\hline DMSO & PC1 500295-52-3 & 0.29 \\
\hline DMSO & PC2 1607469-49-7 & 0.34 \\
\hline DMSO & PC3 870987-63-6 & 0.32 \\
\hline DMSO & PC4 676525-77-2 & 0.37 \\
\hline DMSO & PC5 60804-74-2 & 0.05 \\
\hline DMSO & PC 6 808142-88-3 & 0.35 \\
\hline Dioxane & PC1 500295-52-3 & 0.06 \\
\hline Dioxane & PC2 1607469-49-7 & 0.47 \\
\hline Dioxane & PC3 870987-63-6 & 0.67 \\
\hline Dioxane & PC4 676525-77-2 & 0.20 \\
\hline Dioxane & PC5 60804-74-2 & 0.00 \\
\hline Dioxane & PC 6 808142-88-3 & 0.47 \\
\hline
\end{tabular}


Table S8. Results of High-Throughput Experimentation for Product 16.<smiles>CCOC(=O)c1cnc(S(C)(=O)=O)nc1CNC(=O)OC</smiles>

16

\begin{tabular}{|l|l|l|}
\hline Solvent & Photocatalyst CAS\# & Ratio \\
\hline DMSO & PC1 500295-52-3 & 0.46 \\
\hline DMSO & PC2 1607469-49-7 & 0.41 \\
\hline DMSO & PC3 870987-63-6 & 0.40 \\
\hline DMSO & PC4 676525-77-2 & 0.44 \\
\hline DMSO & PC5 60804-74-2 & 0.19 \\
\hline DMSO & PC 6 808142-88-3 & 0.43 \\
\hline Dioxane & PC1 500295-52-3 & 0.42 \\
\hline Dioxane & PC2 1607469-49-7 & 0.40 \\
\hline Dioxane & PC3 870987-63-6 & 0.75 \\
\hline Dioxane & PC4 676525-77-2 & 0.54 \\
\hline Dioxane & PC5 60804-74-2 & 0.07 \\
\hline Dioxane & PC 6 808142-88-3 & 0.51 \\
\hline
\end{tabular}




\section{Optimization of Reaction Conditions for Alkylation of Halogenated}

\section{Pyrimidines with Redox-Active Esters}

Table S9: Optimization of Reaction Conditions for Alkylation of Pyrimidine 1 with Cyclopentane Redox-Active Ester 2

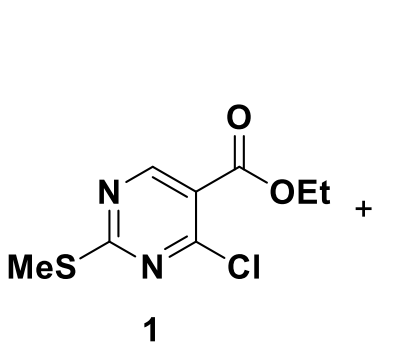

(3.0 equiv)

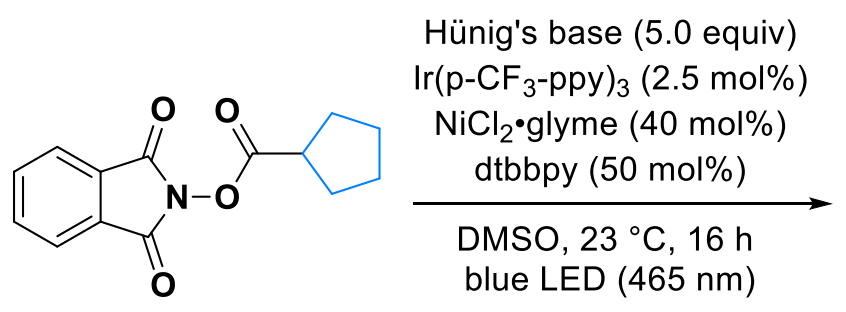

2<smiles>CCOC(=O)c1cnc(S(C)(=O)=O)nc1C1CCCC1</smiles>

3

\begin{tabular}{|c|c|c|}
\hline entry & deviation from above & yield \\
\hline 1 & $\begin{array}{l}1.0 \text { equiv } 1,1.5 \text { equiv } 2,20 \mathrm{~mol} \% \mathrm{Ni}, 25 \mathrm{~mol} \% \text { Ligand, } \\
\left(\operatorname{Ir}\left[\mathrm{df}\left(\mathrm{CF}_{3}\right) \mathrm{ppy}_{2}(\mathrm{dtbpy})\right) \mathrm{PF}_{6} \text { photocatalyst }\right.\end{array}$ & $20 \%$ \\
\hline 2 & 1.0 equiv $1,1.5$ equiv $2,2 \mathrm{~mol} \% \mathrm{Ir}, 20 \mathrm{~mol} \% \mathrm{Ni}, 25 \mathrm{~mol} \%$ Ligand & $27 \%$ \\
\hline 3 & 1.0 equiv $1,1.5$ equiv $2,1 \mathrm{~mol} \% \mathrm{Ir}, 20 \mathrm{~mol} \% \mathrm{Ni}, 25 \mathrm{~mol} \%$ Ligand & $22 \%$ \\
\hline 4 & 1.0 equiv $1,1.5$ equiv $2,5 \mathrm{~mol} \% \mathrm{Ir}, 20 \mathrm{~mol} \% \mathrm{Ni}, 25 \mathrm{~mol} \%$ Ligand & $40 \%$ \\
\hline 5 & 1.0 equiv $1,3.0$ equiv $2,20 \mathrm{~mol} \% \mathrm{Ni}, 25 \mathrm{~mol} \%$ Ligand & $11 \%$ \\
\hline 6 & $20 \mathrm{~mol} \% \mathrm{Ni}, 25 \mathrm{~mol} \%$ Ligand & $58 \%$ \\
\hline 7 & $5 \mathrm{~mol} \% \mathrm{Ir}, 20 \mathrm{~mol} \% \mathrm{Ni}, 25 \mathrm{~mol} \%$ Ligand & $53 \%$ \\
\hline 8 & $1 \mathrm{~mol} \% \mathrm{Ir}, 20 \mathrm{~mol} \% \mathrm{Ni}, 25 \mathrm{~mol} \%$ Ligand & $44 \%$ \\
\hline 9 & $\begin{array}{l}20 \mathrm{~mol} \% \mathrm{Ni}, 25 \mathrm{~mol} \% \text { Ligand, } \\
\text { 1,4-Cyclohexadiene instead of Hünig's base }\end{array}$ & $7 \%$ \\
\hline 10 & 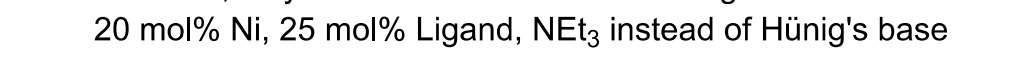 & $48 \%$ \\
\hline 11 & $20 \mathrm{~mol} \% \mathrm{Ni}, 25 \mathrm{~mol} \%$ Ligand, Hantzsch ester instead of Hünig's base & $11 \%$ \\
\hline 12 & $20 \mathrm{~mol} \% \mathrm{Ni}, 25 \mathrm{~mol} \%$ Ligand, 1,4-Cyclohexadiene (1.25 equiv) & $60 \%$ \\
\hline 13 & $20 \mathrm{~mol} \% \mathrm{Ni}, 25 \mathrm{~mol} \%$ Ligand, $\mathrm{NEt}_{3}$ (1.25 equiv) & $45 \%$ \\
\hline 14 & $20 \mathrm{~mol} \% \mathrm{Ni}, 25 \mathrm{~mol} \%$ Ligand, Hantzsch ester ( 1.25 equiv) & $48 \%$ \\
\hline 15 & $20 \mathrm{~mol} \% \mathrm{Ni}, 25 \mathrm{~mol} \%$ Ligand, 8.0 equivalents Hünig's base & $15 \%$ \\
\hline 16 & 8.0 equivalents Hünig's base & $28 \%$ \\
\hline 17 & Zn (3.0 equiv) instead of Hünig's Base & $22 \%$ \\
\hline 18 & $\mathrm{Zn}$ (3.0 equiv) and $\mathrm{LiCl}$ (3.0 equiv) instead of Hünig's Base & $17 \%$ \\
\hline 19 & No $\mathrm{Ni}$, Ligand & $6 \%$ \\
\hline 20 & No Ir, $20 \mathrm{~mol} \% \mathrm{Ni}, 25 \mathrm{~mol} \%$ Ligand & $<5 \%$ \\
\hline 21 & No Hünig's base, $20 \mathrm{~mol} \% \mathrm{Ni}, 25 \mathrm{~mol} \%$ Ligand & $15 \%$ \\
\hline 22 & $5 \mathrm{~mol} \% \mathrm{Ir}$ & $\begin{array}{l}64 \% \\
16 \%\end{array}$ \\
\hline 23 & 1 hour reaction time & $16 \%$ \\
\hline 24 & 5 hour reaction time & $45 \%$ \\
\hline 25 & 1,4-dioxane instead of DMSO & $40 \%$ \\
\hline 26 & No blue LED & $0 \%$ \\
\hline 27 & None & $70 \%$ \\
\hline
\end{tabular}


Table S10: Optimization of Reaction Conditions for Alkylation of Pyrimidine 1 with Cyclobutane Redox-Active Ester 46

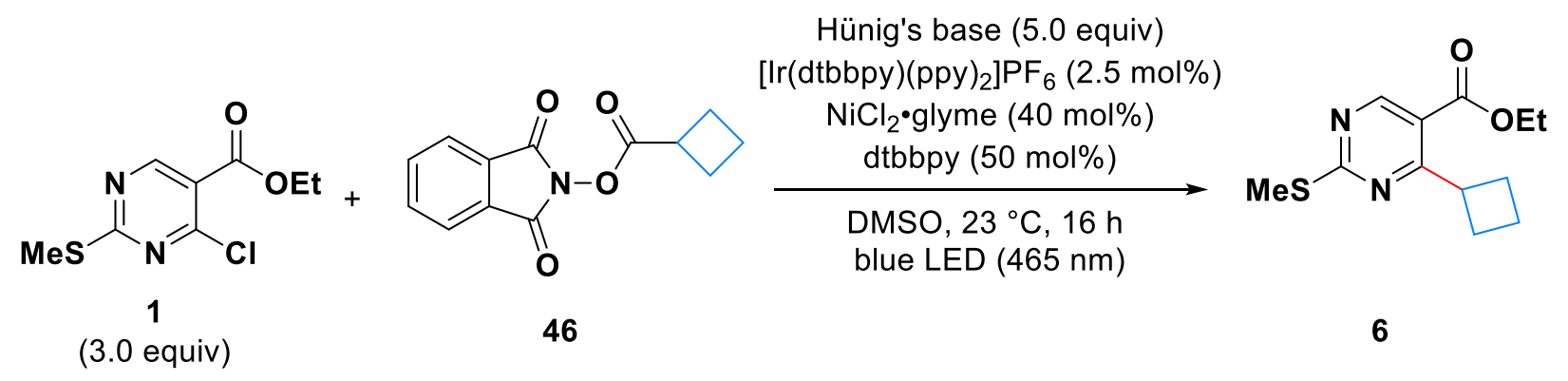

\begin{tabular}{ccc}
\hline entry & deviation from above & yield \\
\hline 1 & 1.0 equiv 1, 1.5 equiv 46, 20 mol\% Ni, 25 mol\% Ligand & $12 \%$ \\
2 & $20 \mathrm{~mol} \% \mathrm{Ni}, 25 \mathrm{~mol} \%$ Ligand & $36 \%$ \\
3 & DMSO instead of 1,4-dioxane & $47 \%$ \\
4 & None & $58 \%$
\end{tabular}


NMR Characterization 


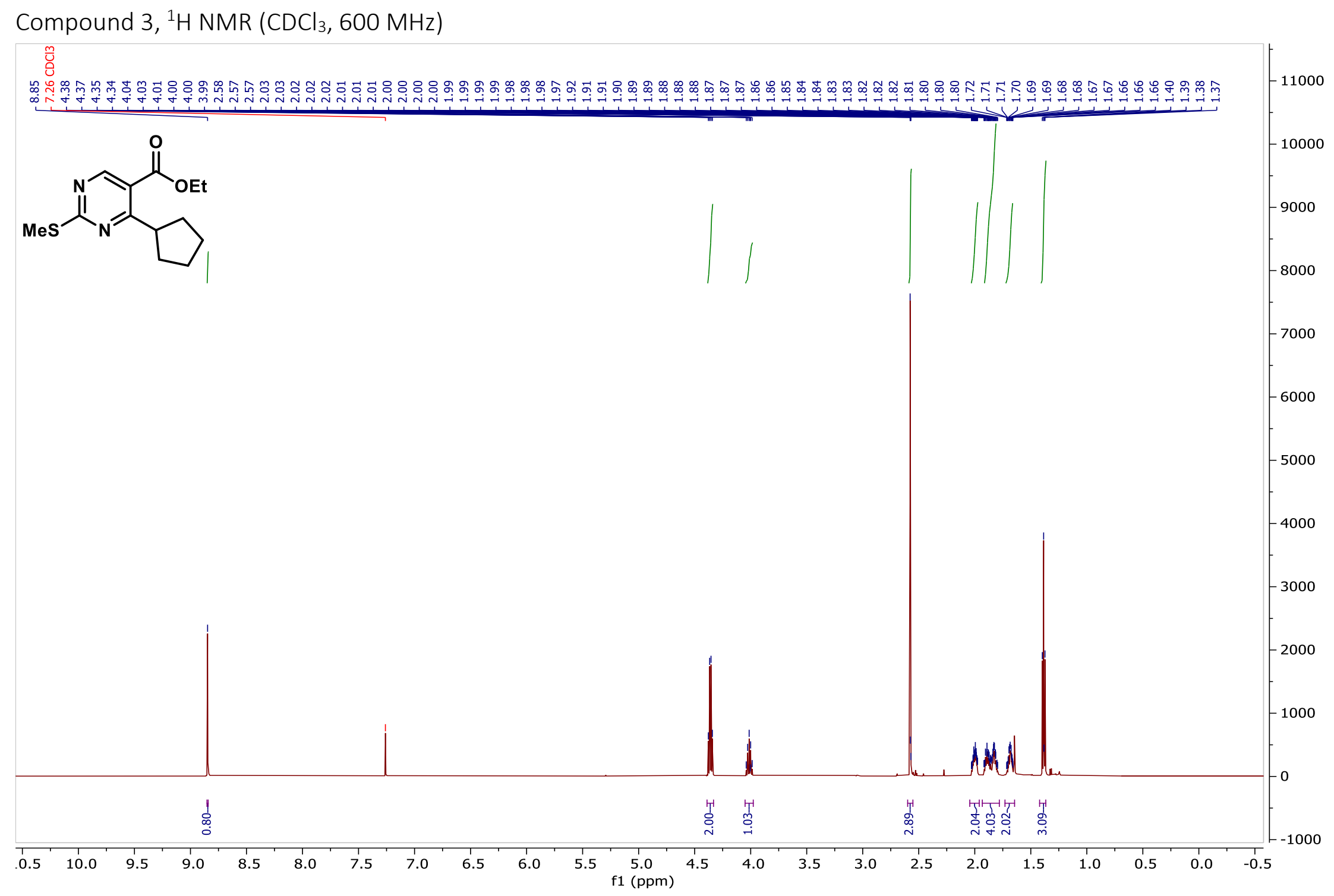


Compound $3,{ }^{13} \mathrm{C}\left\{{ }^{1} \mathrm{H}\right\} \mathrm{NMR}\left(\mathrm{CDCl}_{3}, 151 \mathrm{MHz}\right)$

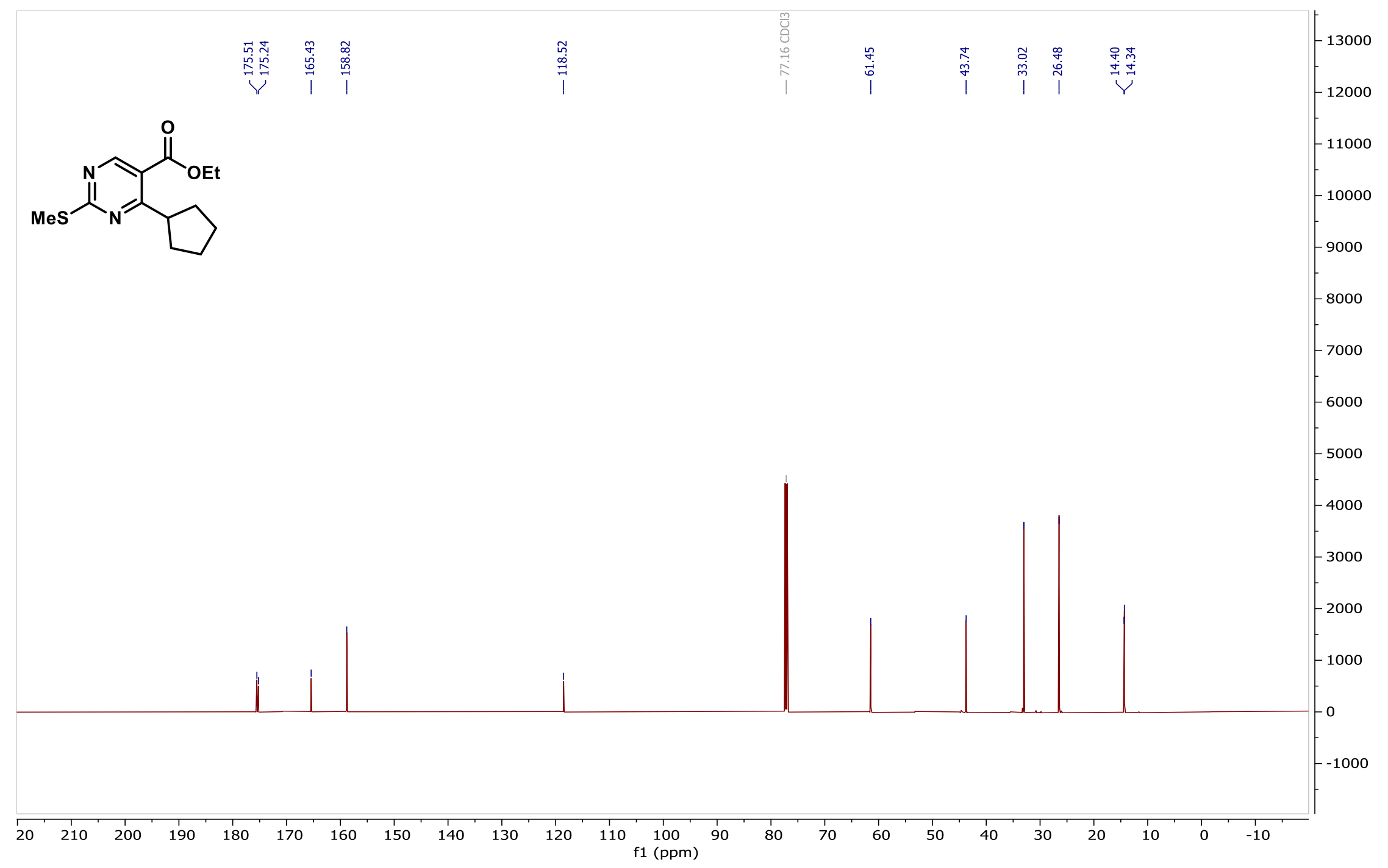


Compound 6, ${ }^{1} \mathrm{H} \mathrm{NMR}\left(\mathrm{CDCl}_{3}, 600 \mathrm{MHz}\right)$

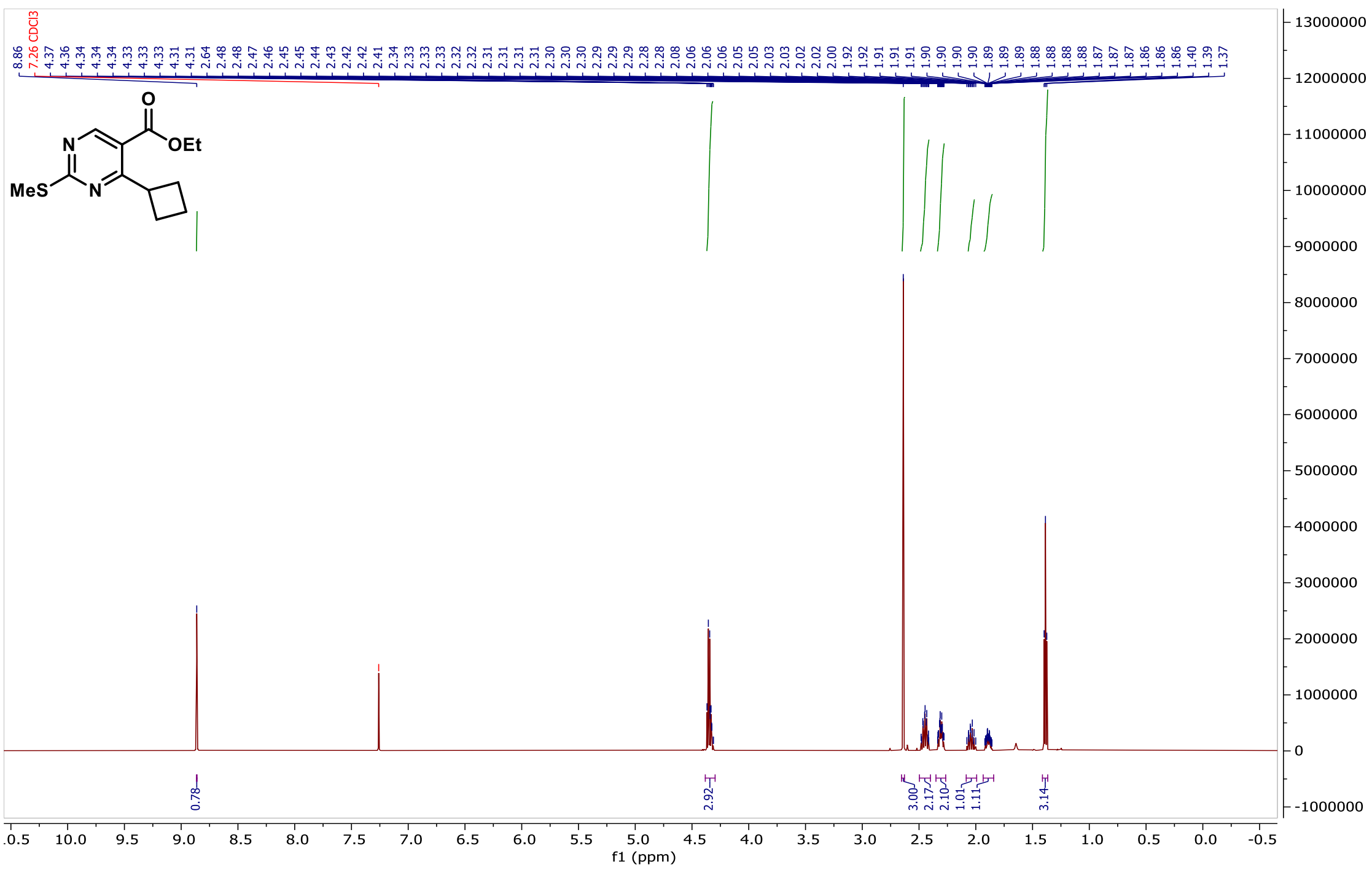


Compound $6,{ }^{13} \mathrm{C}\left\{{ }^{1} \mathrm{H}\right\} \mathrm{NMR}\left(\mathrm{CDCl}_{3}, 151 \mathrm{MHz}\right)$

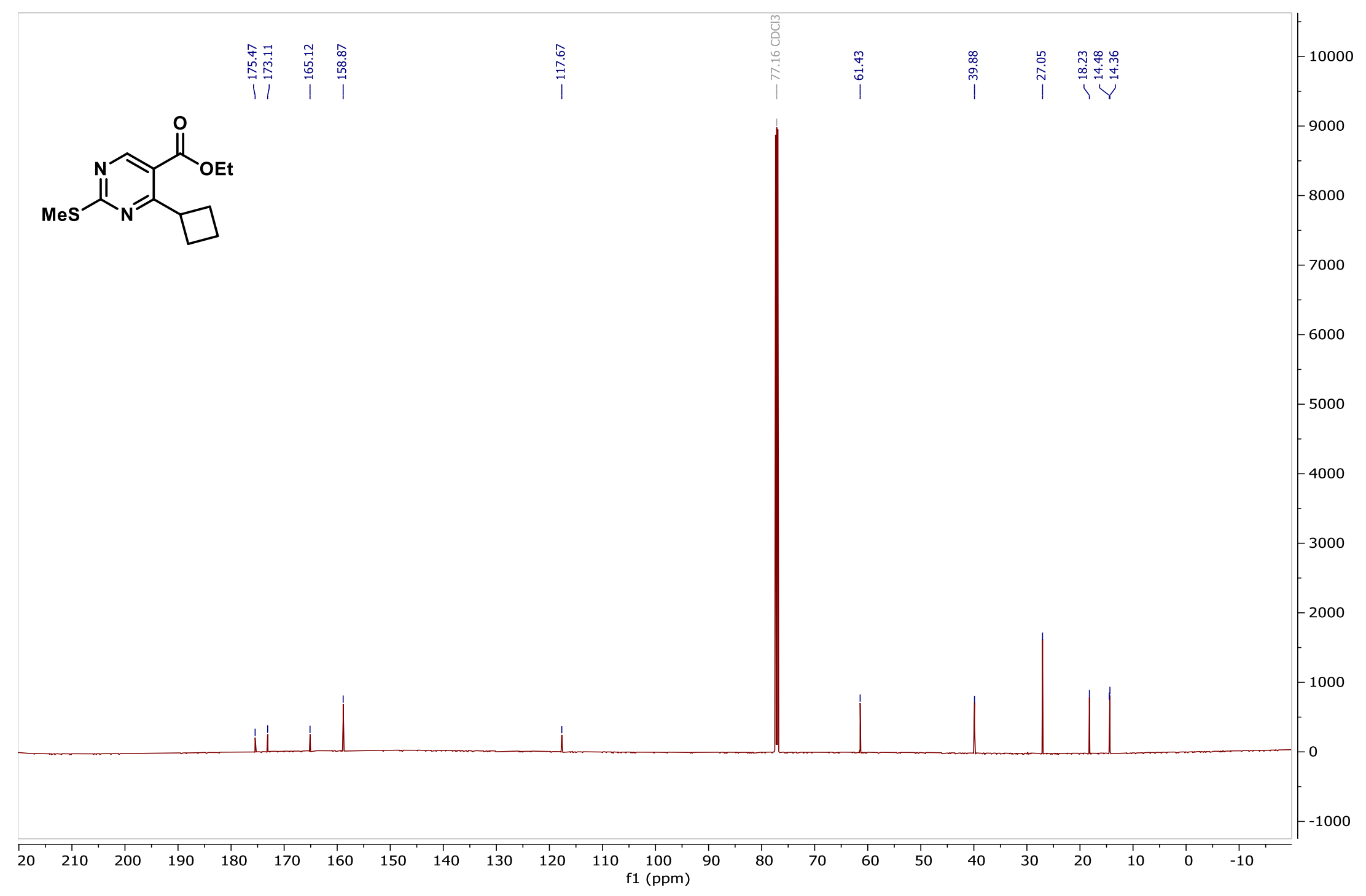


Compound $7,{ }^{1} \mathrm{H}$ NMR $\left(\mathrm{CDCl}_{3}, 600 \mathrm{MHz}\right)$

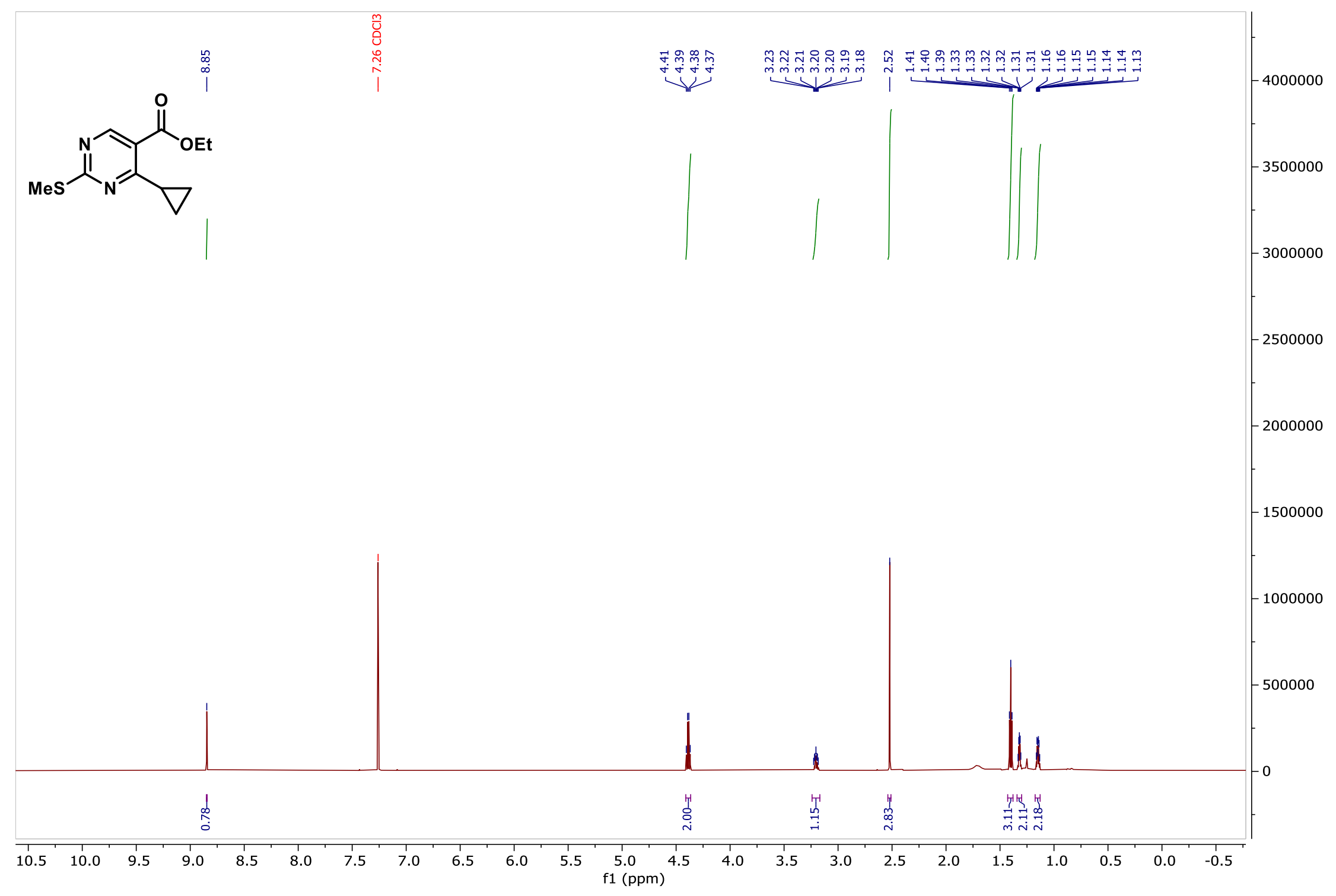


Compound $7,{ }^{13} \mathrm{C}\left\{{ }^{1} \mathrm{H}\right\} \mathrm{NMR}\left(\mathrm{CDCl}_{3}, 151 \mathrm{MHz}\right)$

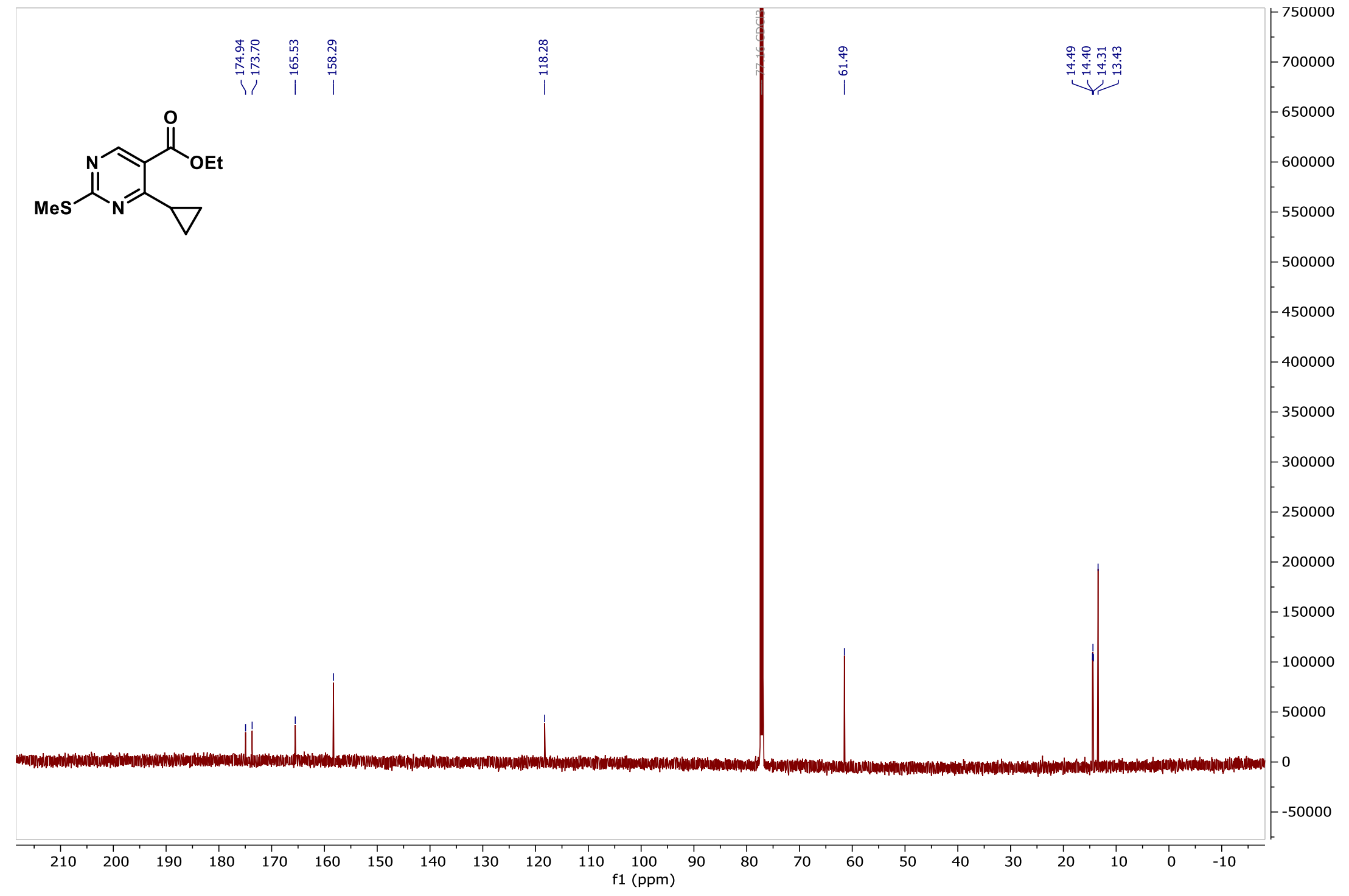


Compound 8, ${ }^{1} \mathrm{H} \mathrm{NMR}\left(\mathrm{CDCl}_{3}, 600 \mathrm{MHz}\right)$

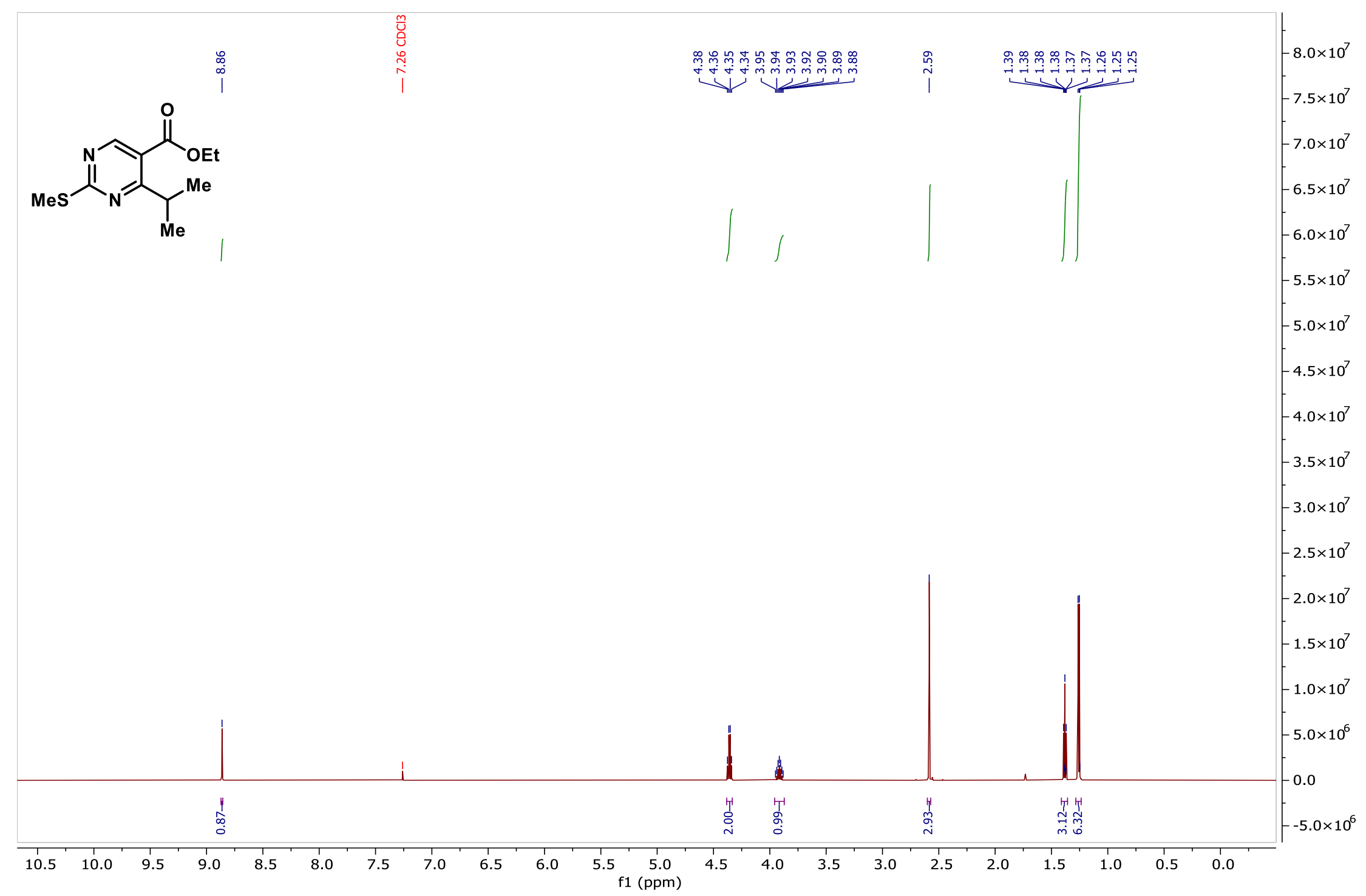


Compound $8,{ }^{13} \mathrm{C}\left\{{ }^{1} \mathrm{H}\right\} \mathrm{NMR}\left(\mathrm{CDCl}_{3}, 151 \mathrm{MHz}\right)$

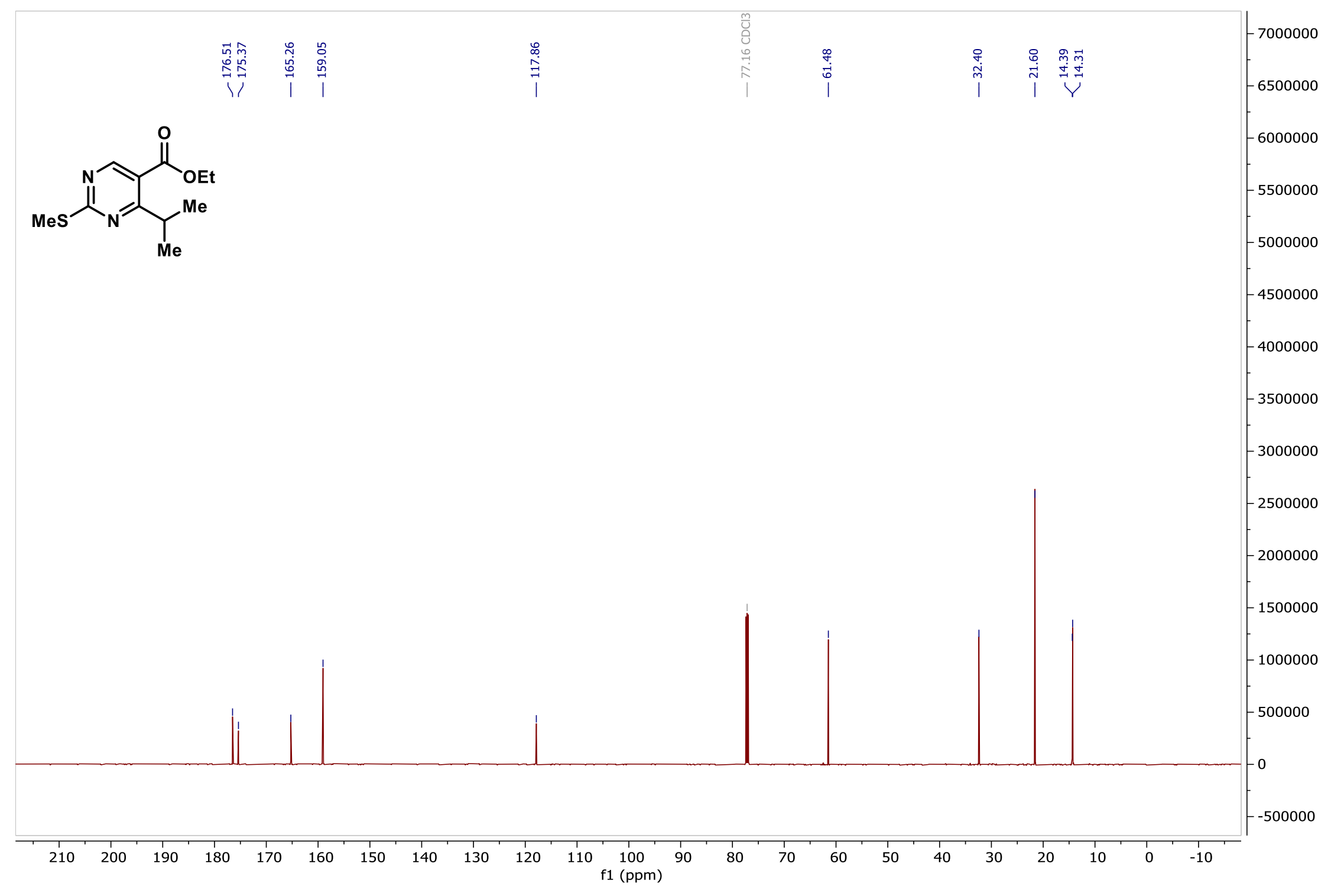


Compound 9, ${ }^{1} \mathrm{H} \mathrm{NMR}\left(\mathrm{CDCl}_{3}, 600 \mathrm{MHz}\right)$

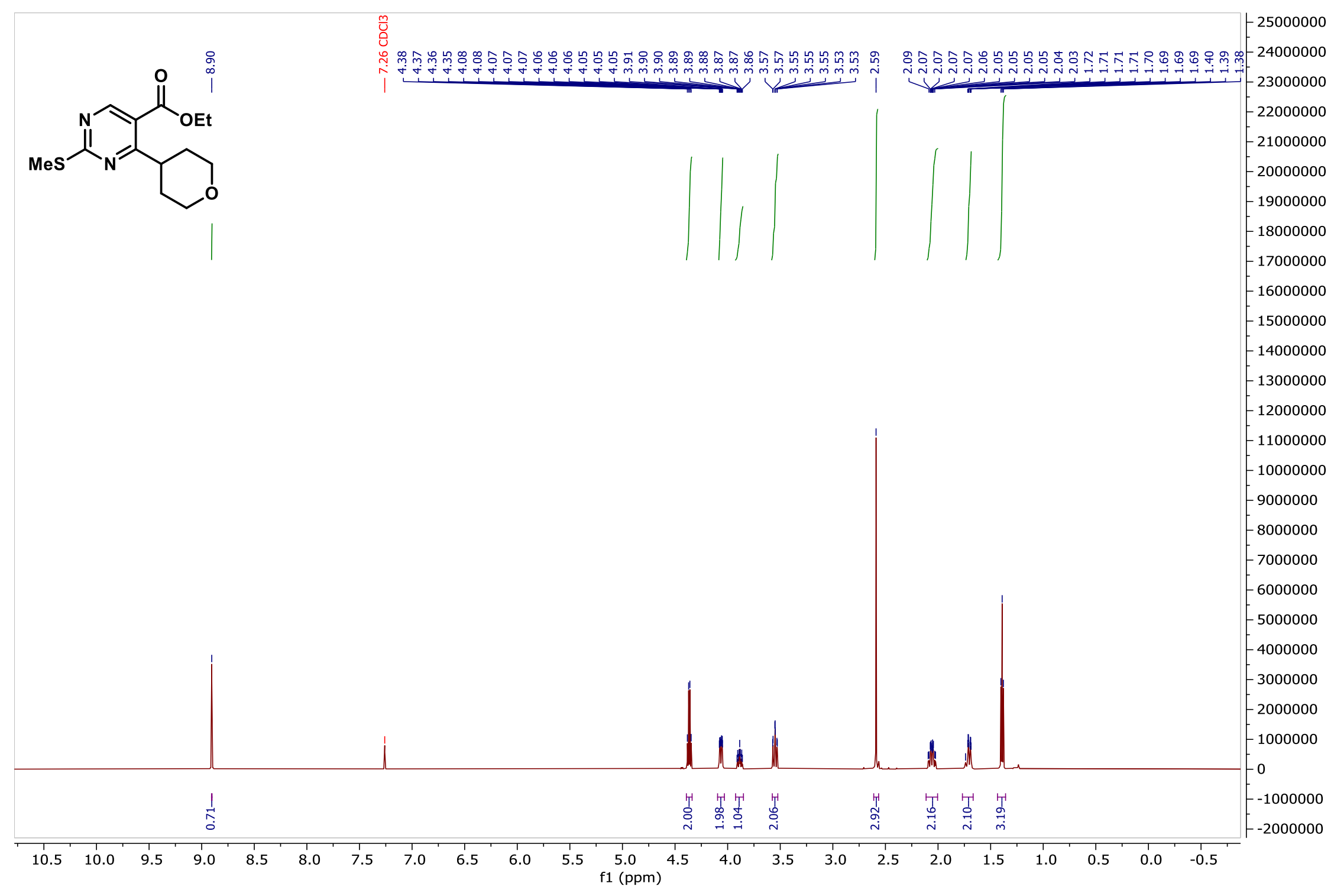


Compound $9,{ }^{13} \mathrm{C}\left\{{ }^{1} \mathrm{H}\right\} \mathrm{NMR}\left(\mathrm{CDCl}_{3}, 151 \mathrm{MHz}\right)$

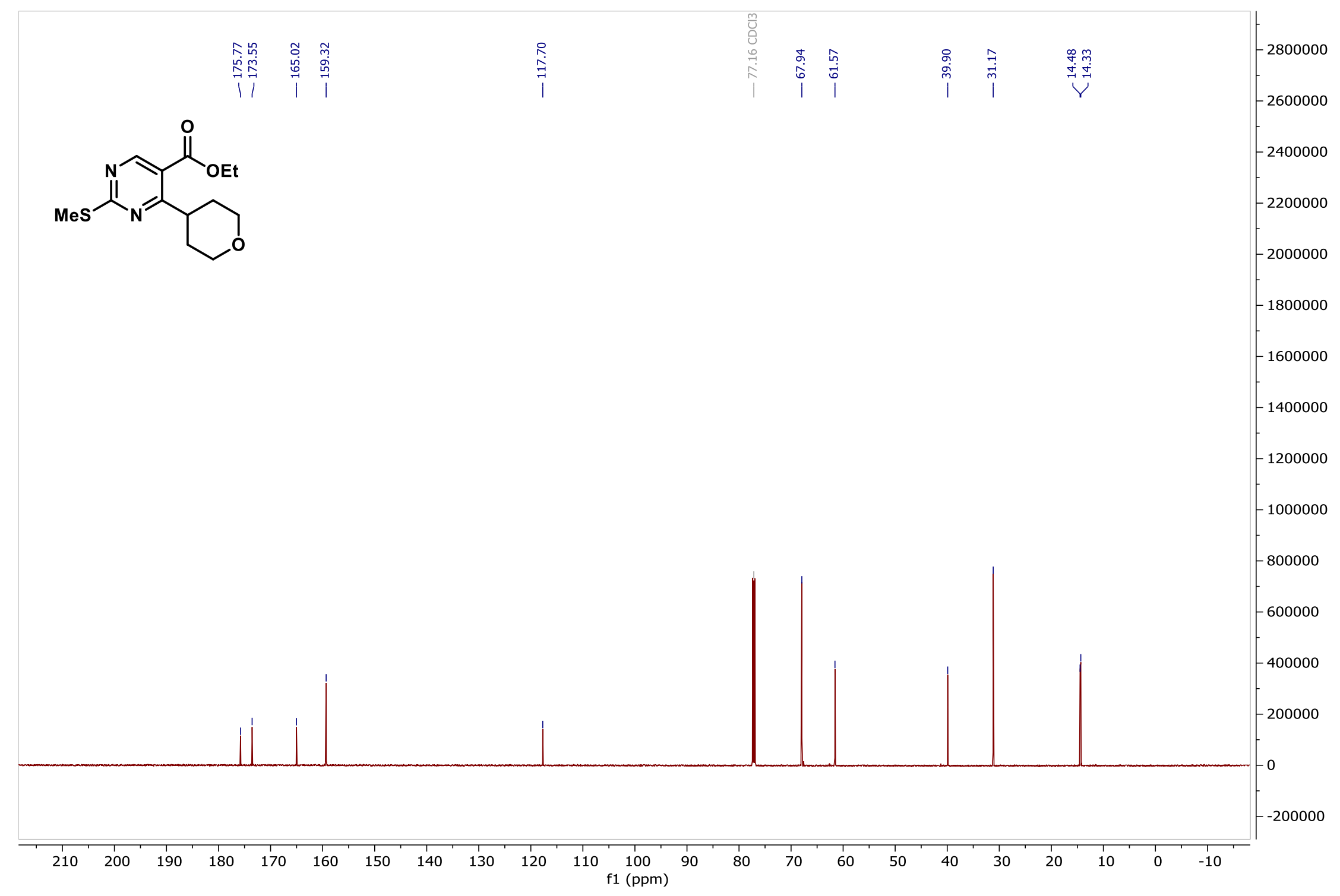


Compound 10, ${ }^{1} \mathrm{H}$ NMR $\left(\mathrm{CDCl}_{3}, 600 \mathrm{MHz}\right)$

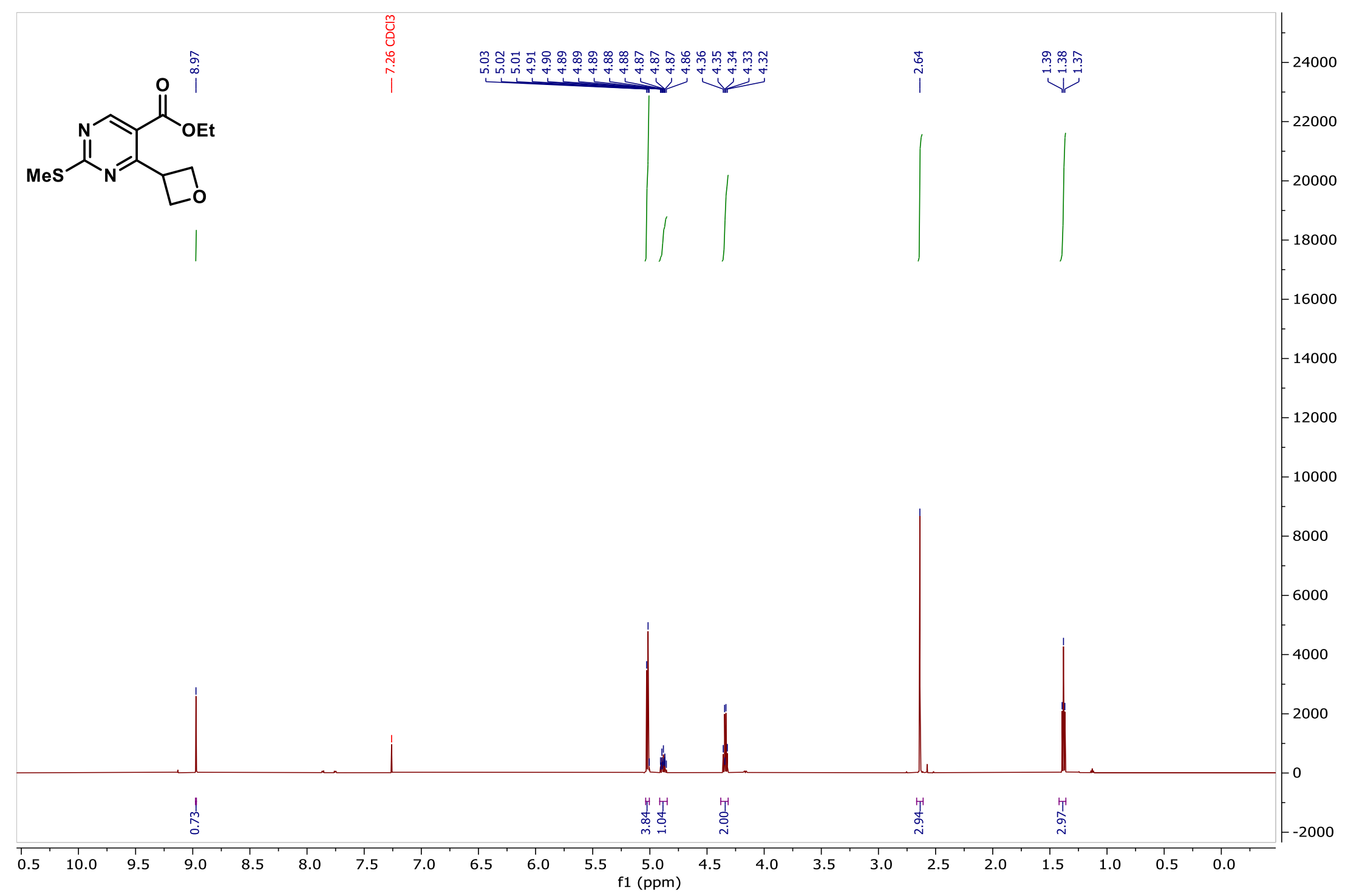


Compound $10,{ }^{13} \mathrm{C}\left\{{ }^{1} \mathrm{H}\right\} \operatorname{NMR}\left(\mathrm{CDCl}_{3}, 151 \mathrm{MHz}\right)$

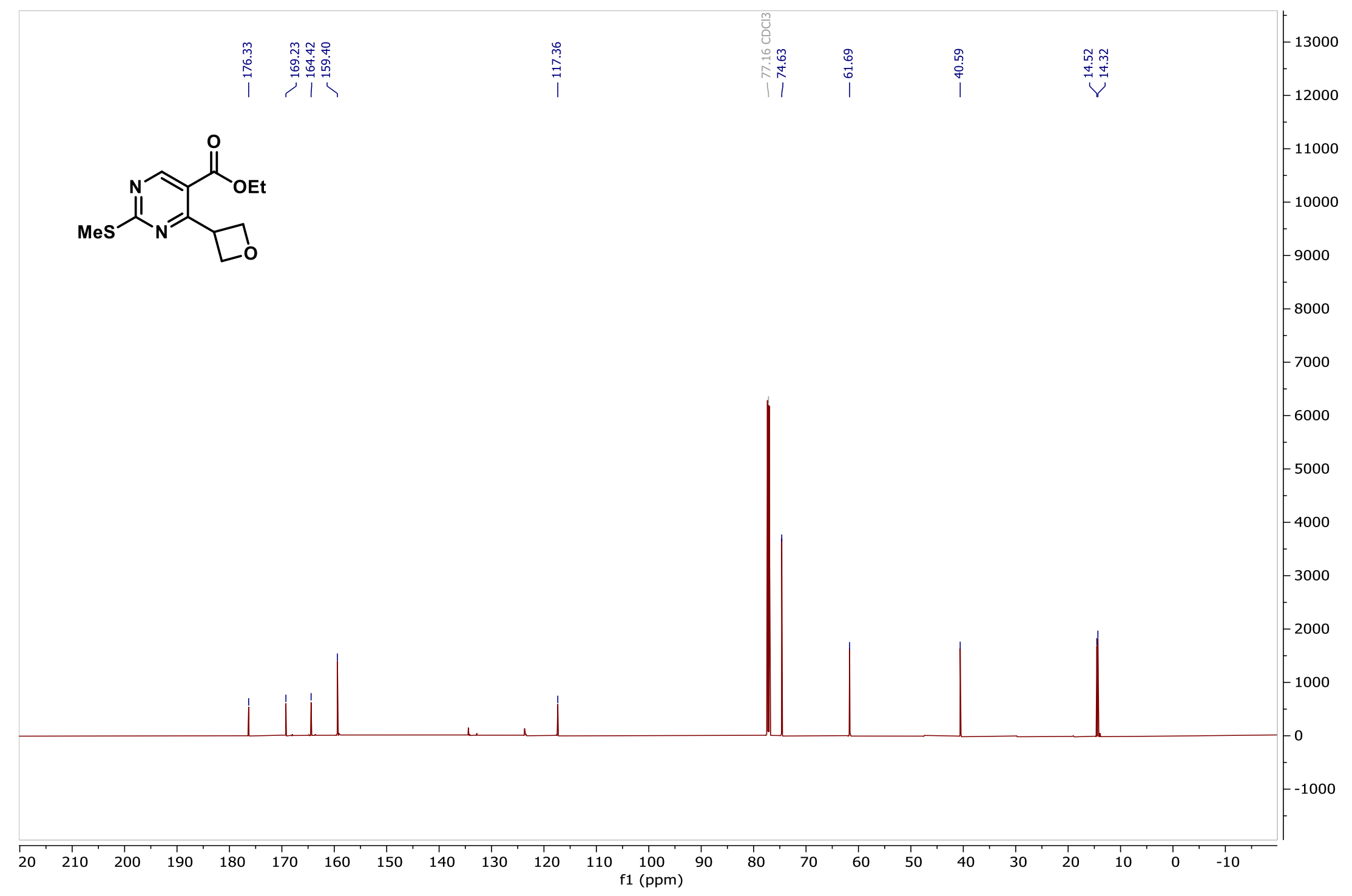


Compound $11,{ }^{1} \mathrm{H}$ NMR $\left(\mathrm{CDCl}_{3}, 600 \mathrm{MHz}\right)$

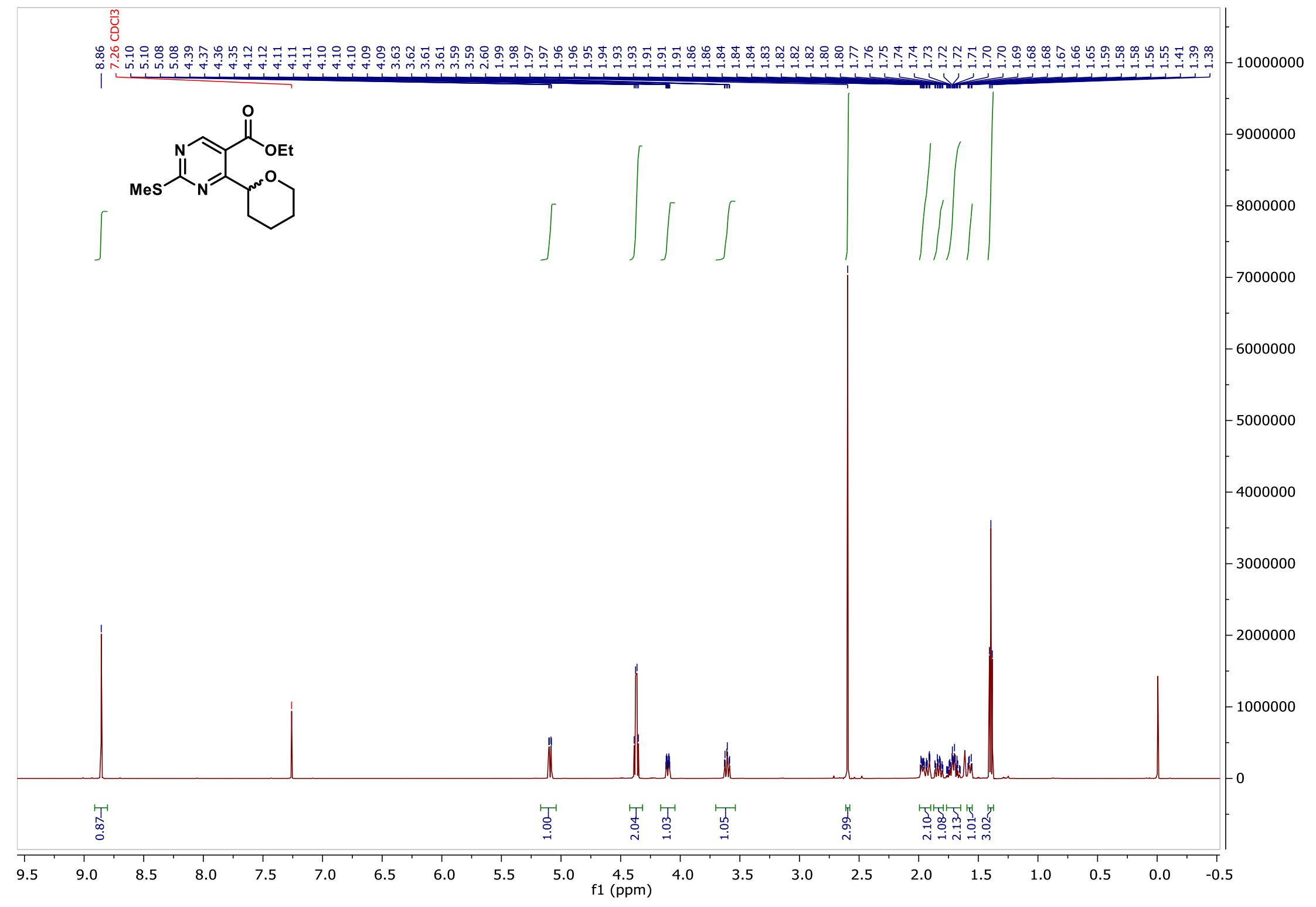


Compound $11,{ }^{13} \mathrm{C}\left\{{ }^{1} \mathrm{H}\right\}$ NMR $\left(\mathrm{CDCl}_{3}, 151 \mathrm{MHz}\right)$

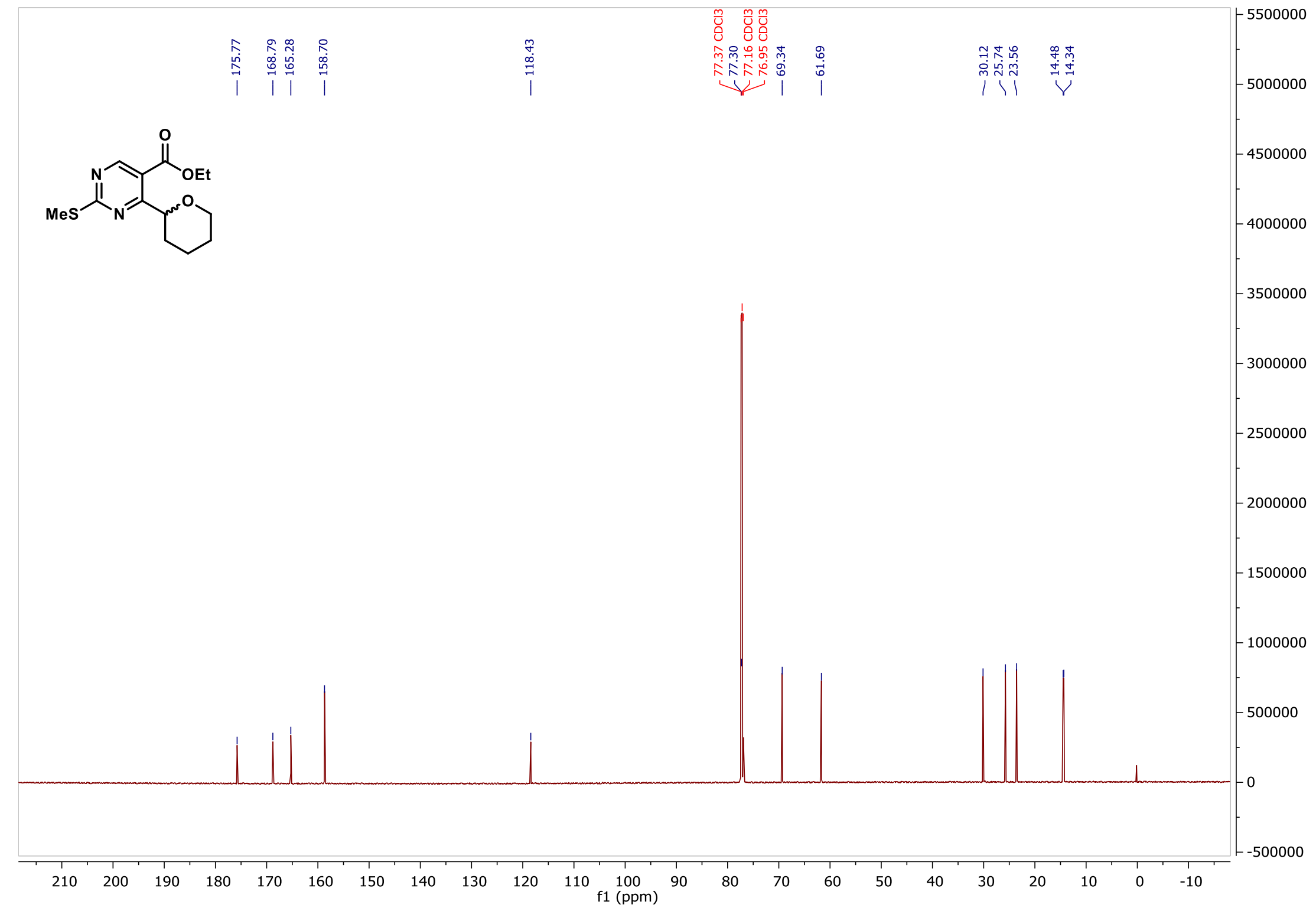


Compound $12,{ }^{1} \mathrm{H}$ NMR $\left(\mathrm{CDCl}_{3}, 600 \mathrm{MHz}\right)$

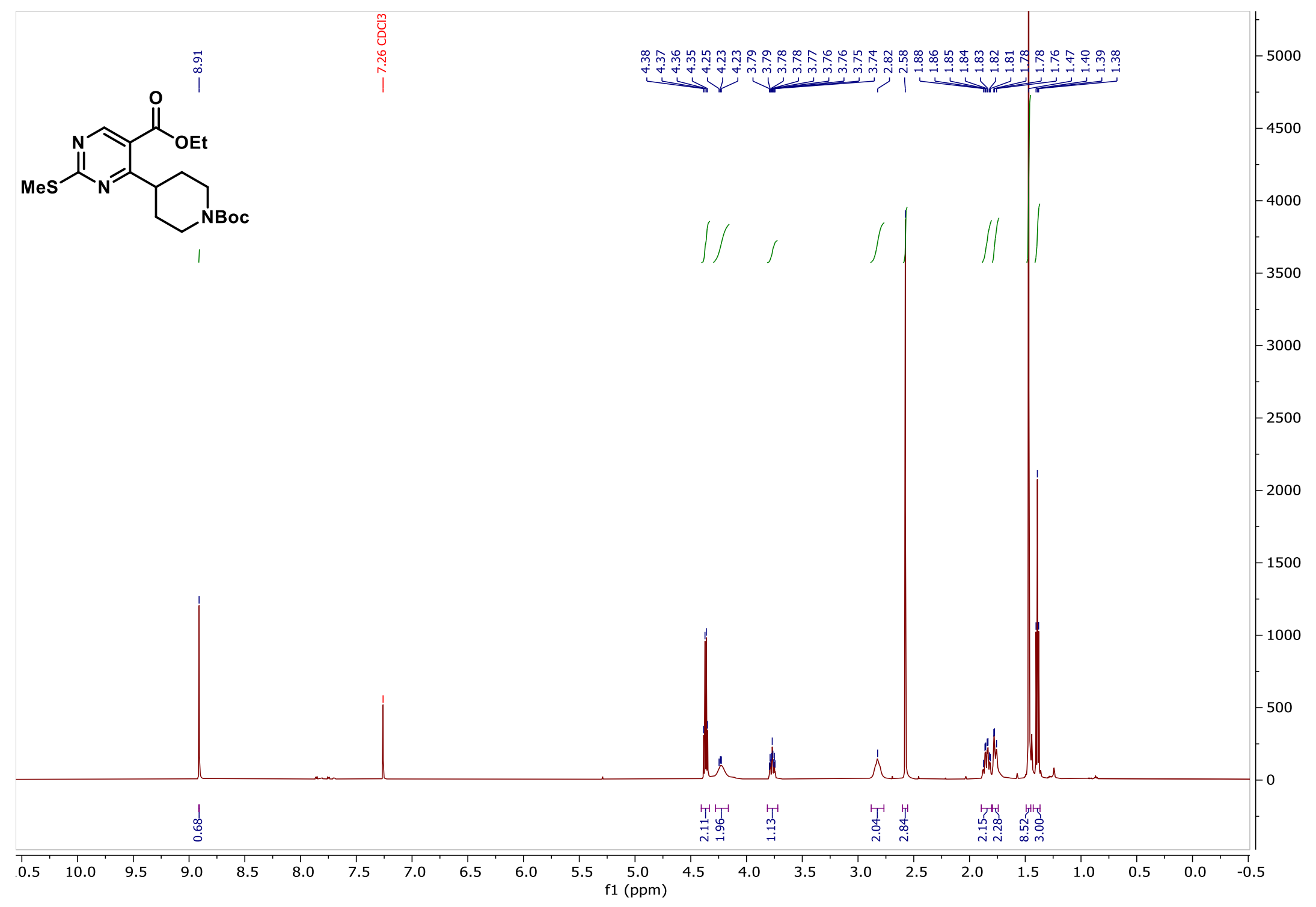


Compound $12,{ }^{13} \mathrm{C}\left\{{ }^{1} \mathrm{H}\right\}$ NMR $\left(\mathrm{CDCl}_{3}, 151 \mathrm{MHz}\right)$

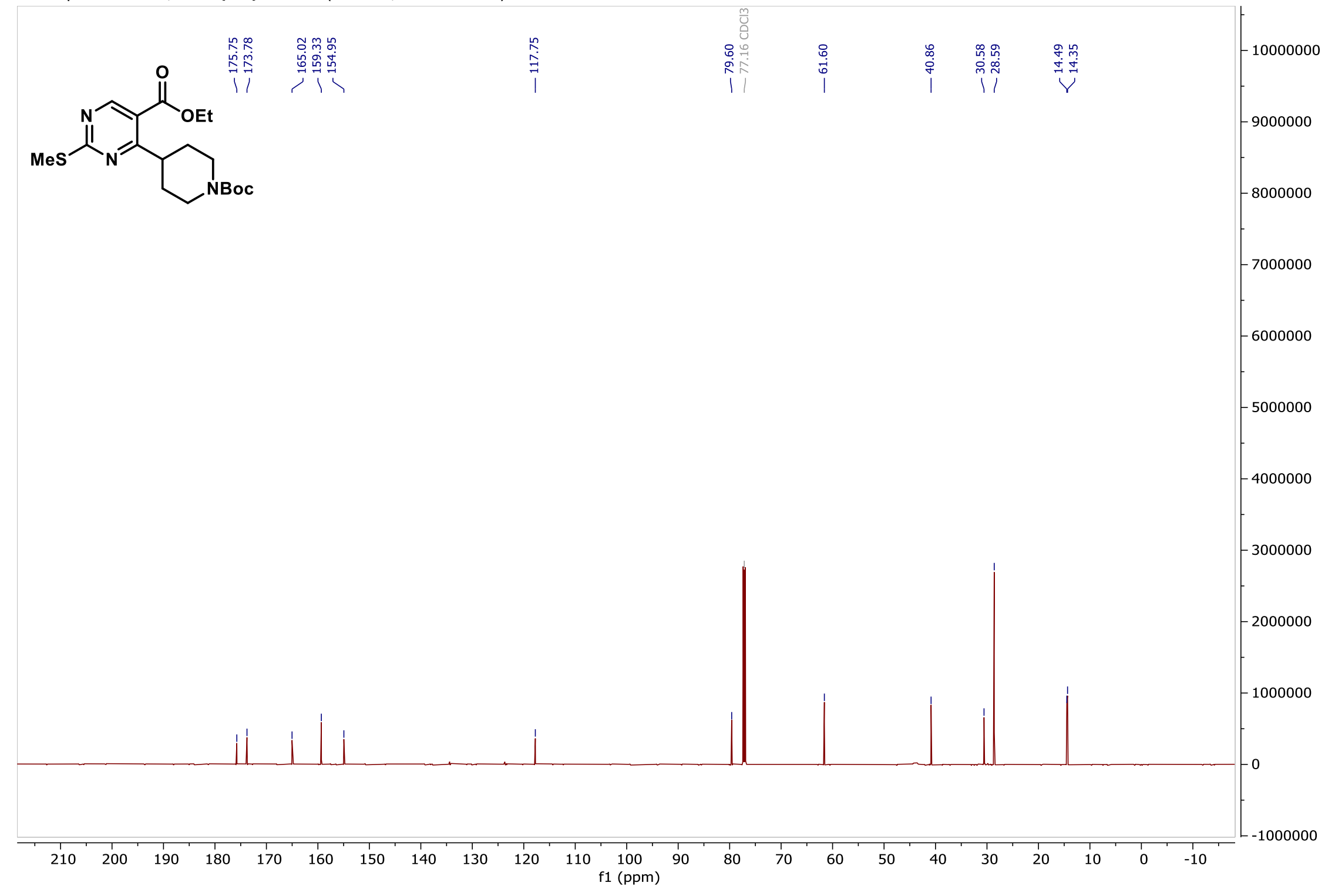


Compound $13,{ }^{1} \mathrm{H} \mathrm{NMR}\left(\mathrm{CDCl}_{3}, 400 \mathrm{MHz}\right)$

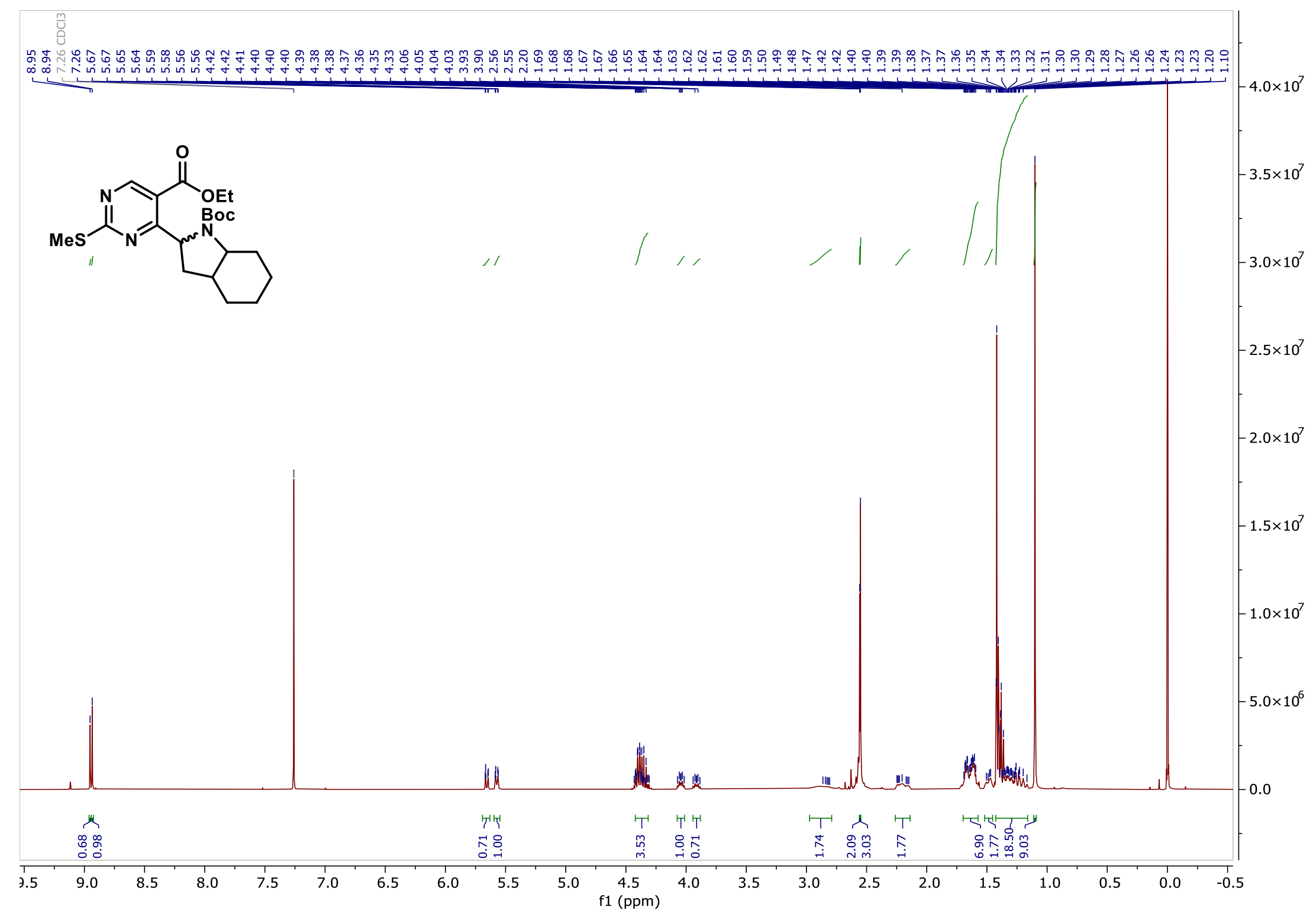




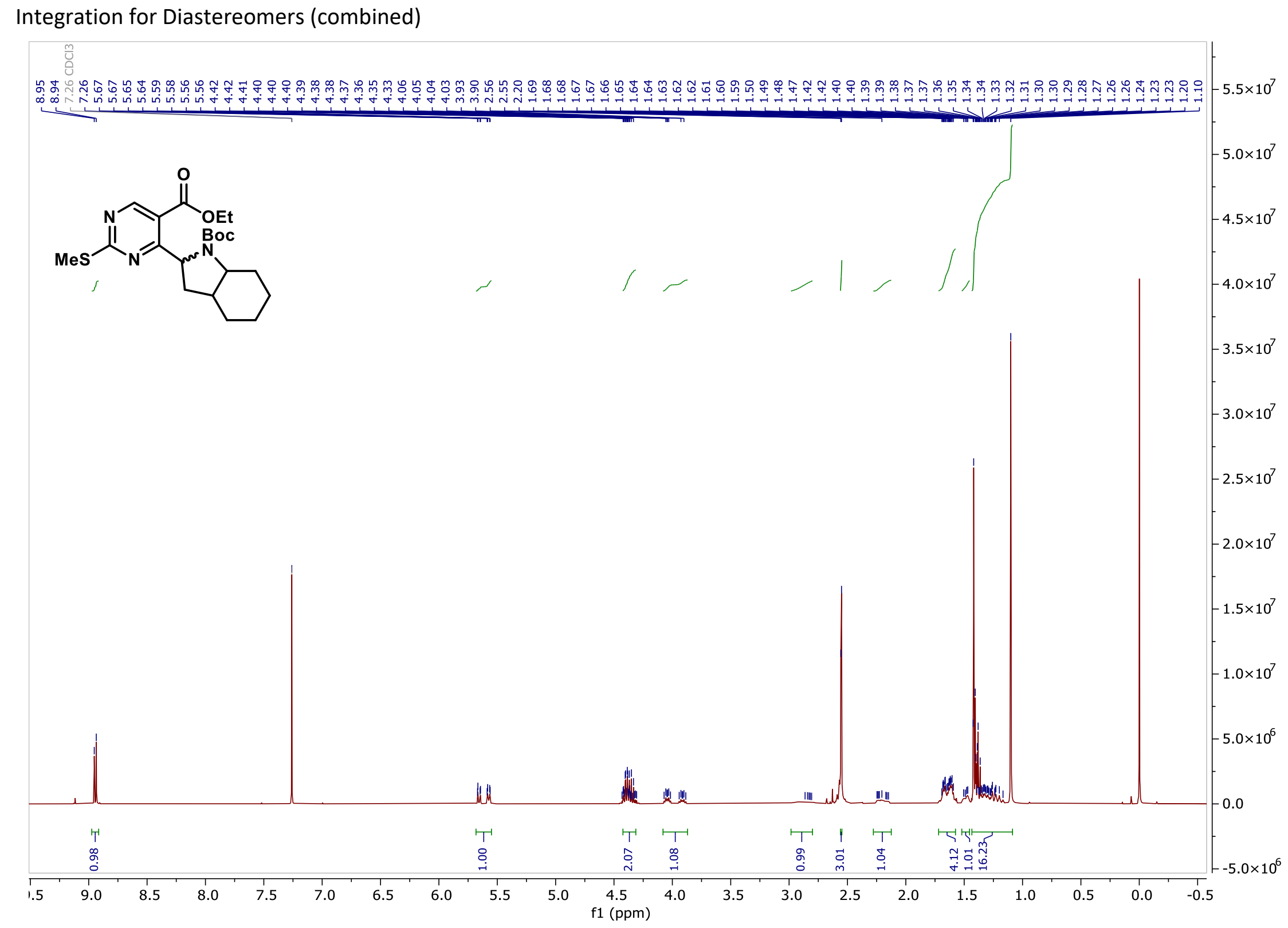


Compound $13,{ }^{13} \mathrm{C}\left\{{ }^{1} \mathrm{H}\right\}$ NMR $\left(\mathrm{CDCl}_{3}, 101 \mathrm{MHz}\right)$

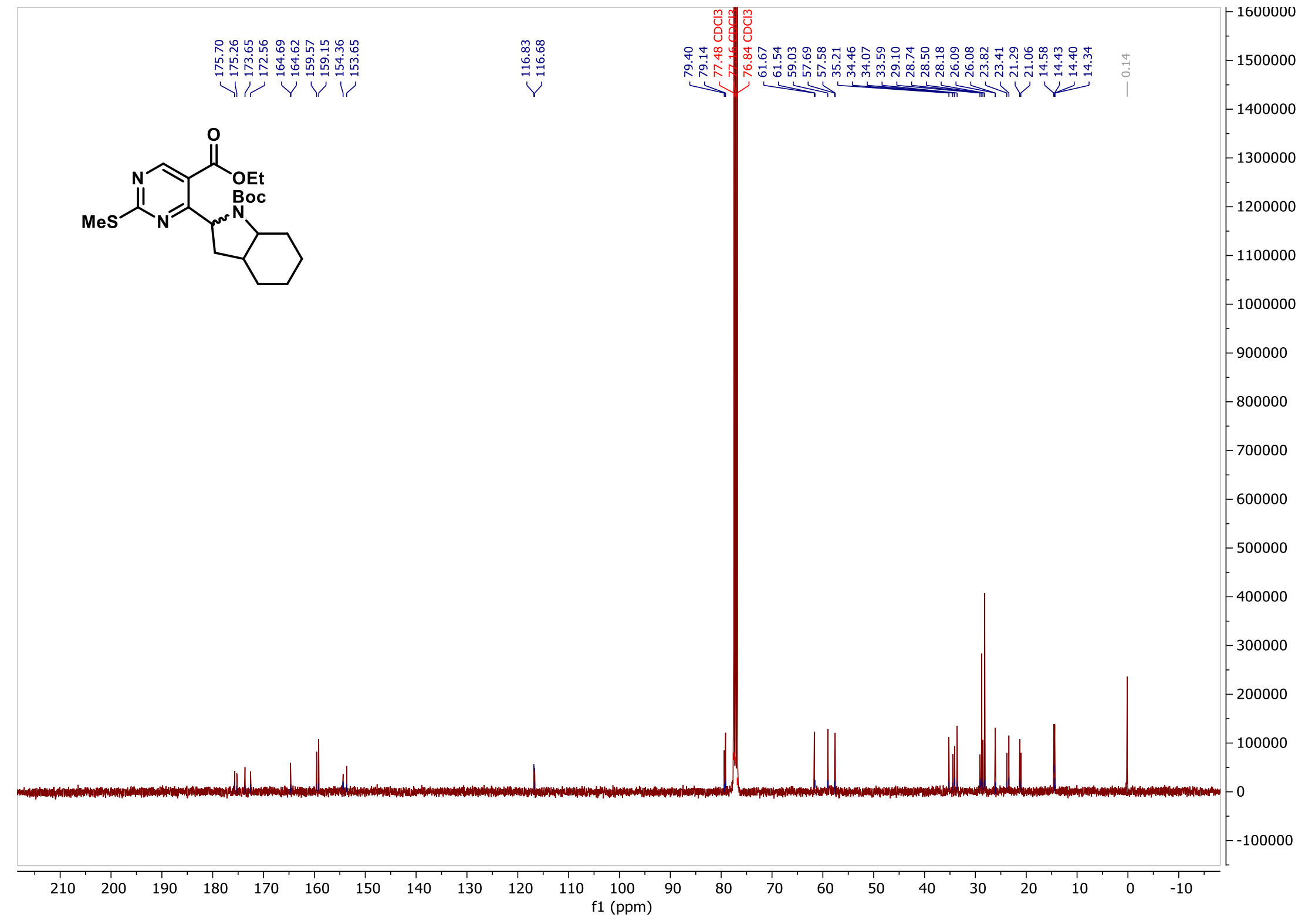


Compound $14,{ }^{1} \mathrm{H}$ NMR $\left(\mathrm{CDCl}_{3}, 600 \mathrm{MHz}\right)$

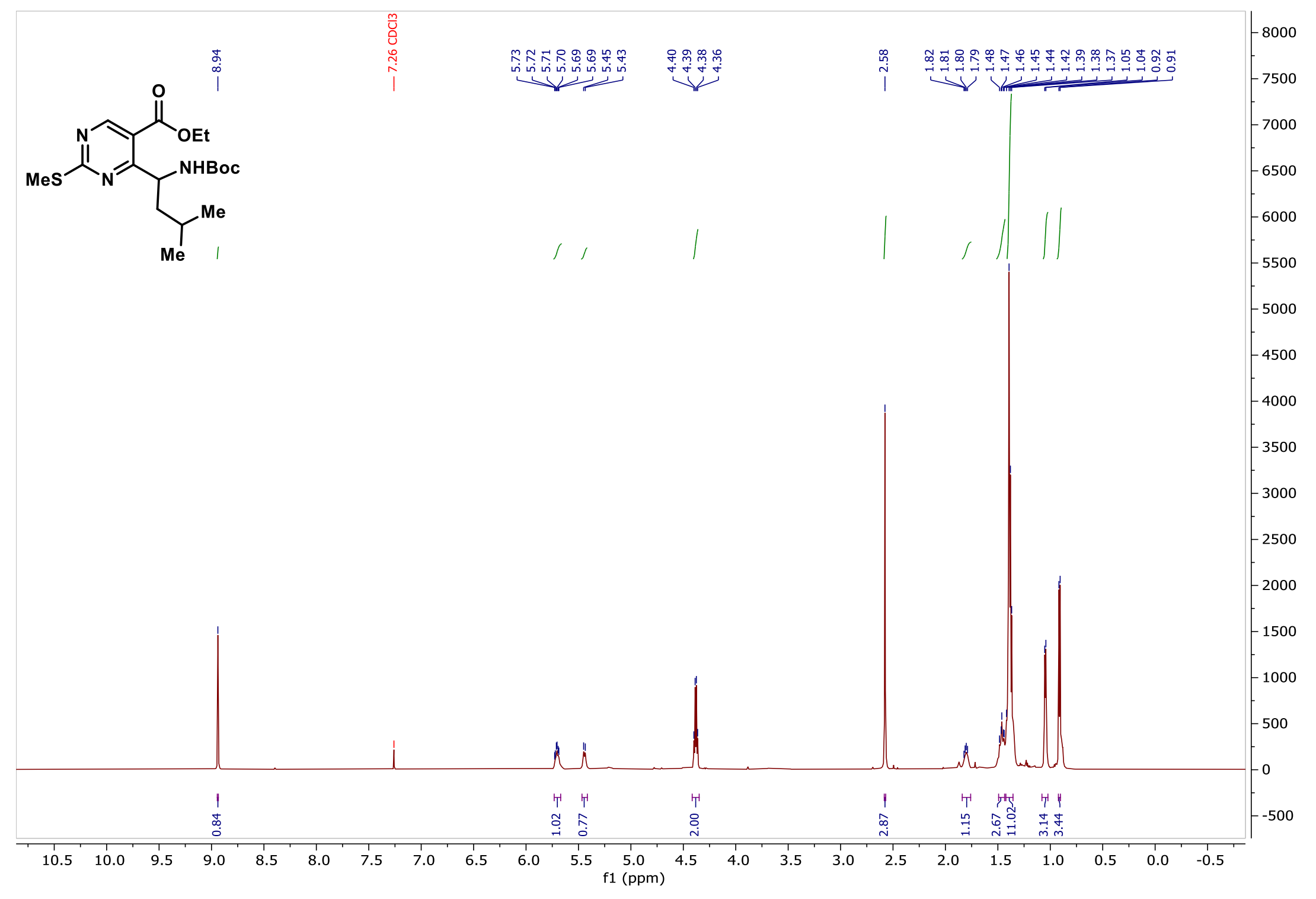


Compound $14,{ }^{13} \mathrm{C}\left\{{ }^{1} \mathrm{H}\right\}$ NMR $\left(\mathrm{CDCl}_{3}, 151 \mathrm{MHz}\right)$

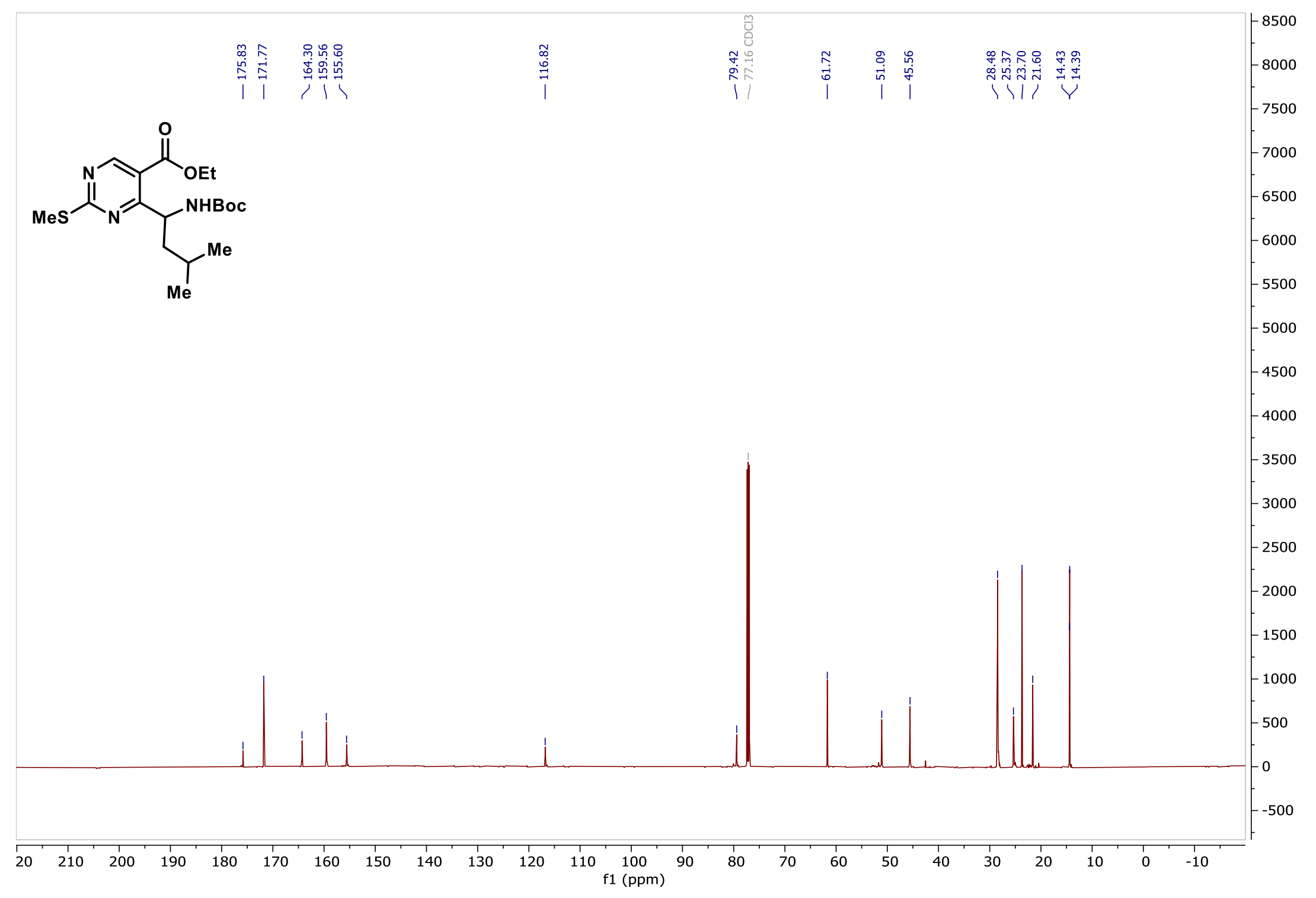


Compound 15, ${ }^{1} \mathrm{H}$ NMR (CDCl $\left.3,600 \mathrm{MHz}\right)$

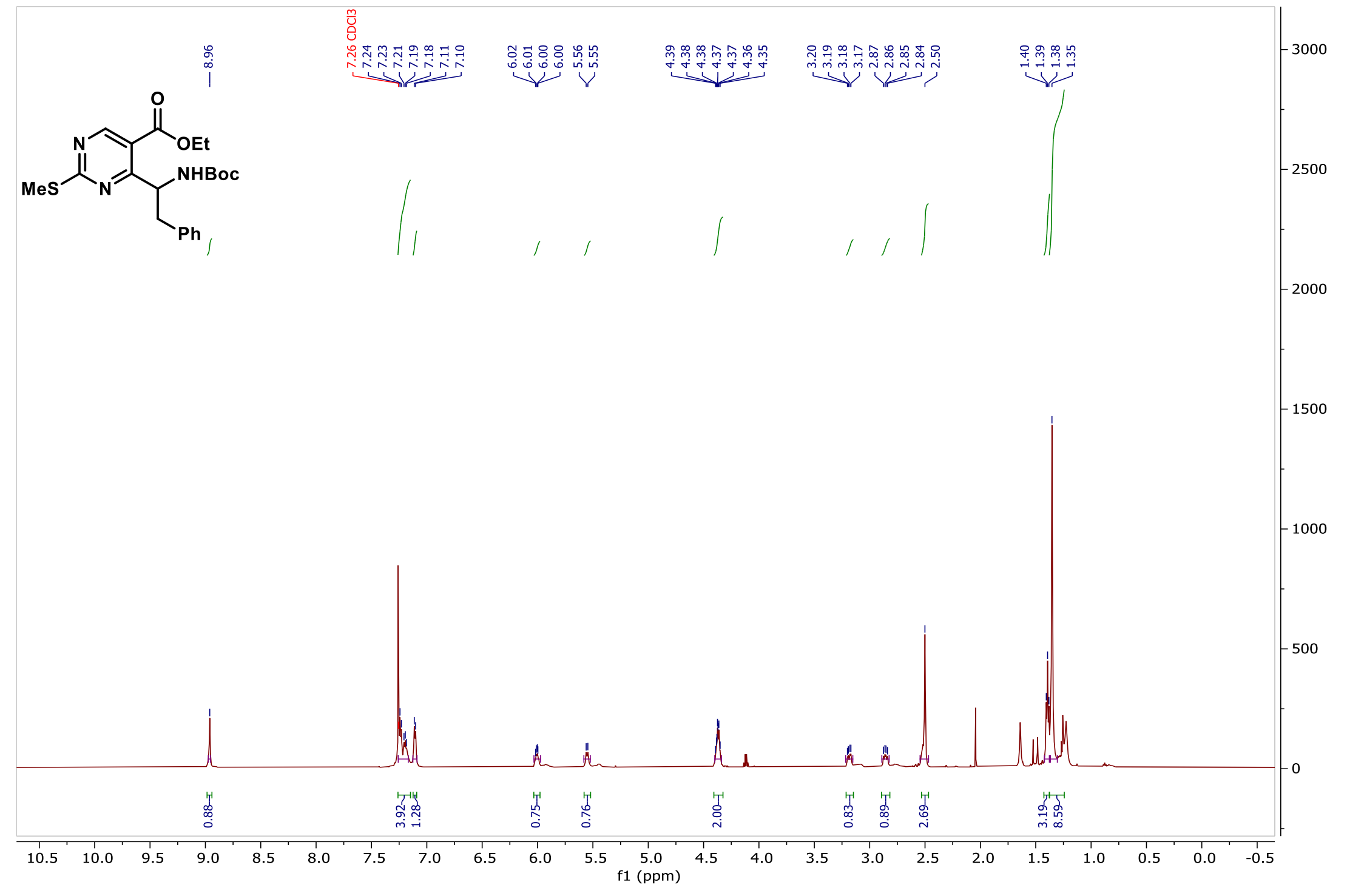


Compound $15,{ }^{13} \mathrm{C}\left\{{ }^{1} \mathrm{H}\right\}$ NMR $\left(\mathrm{CDCl}_{3}, 151 \mathrm{MHz}\right)$

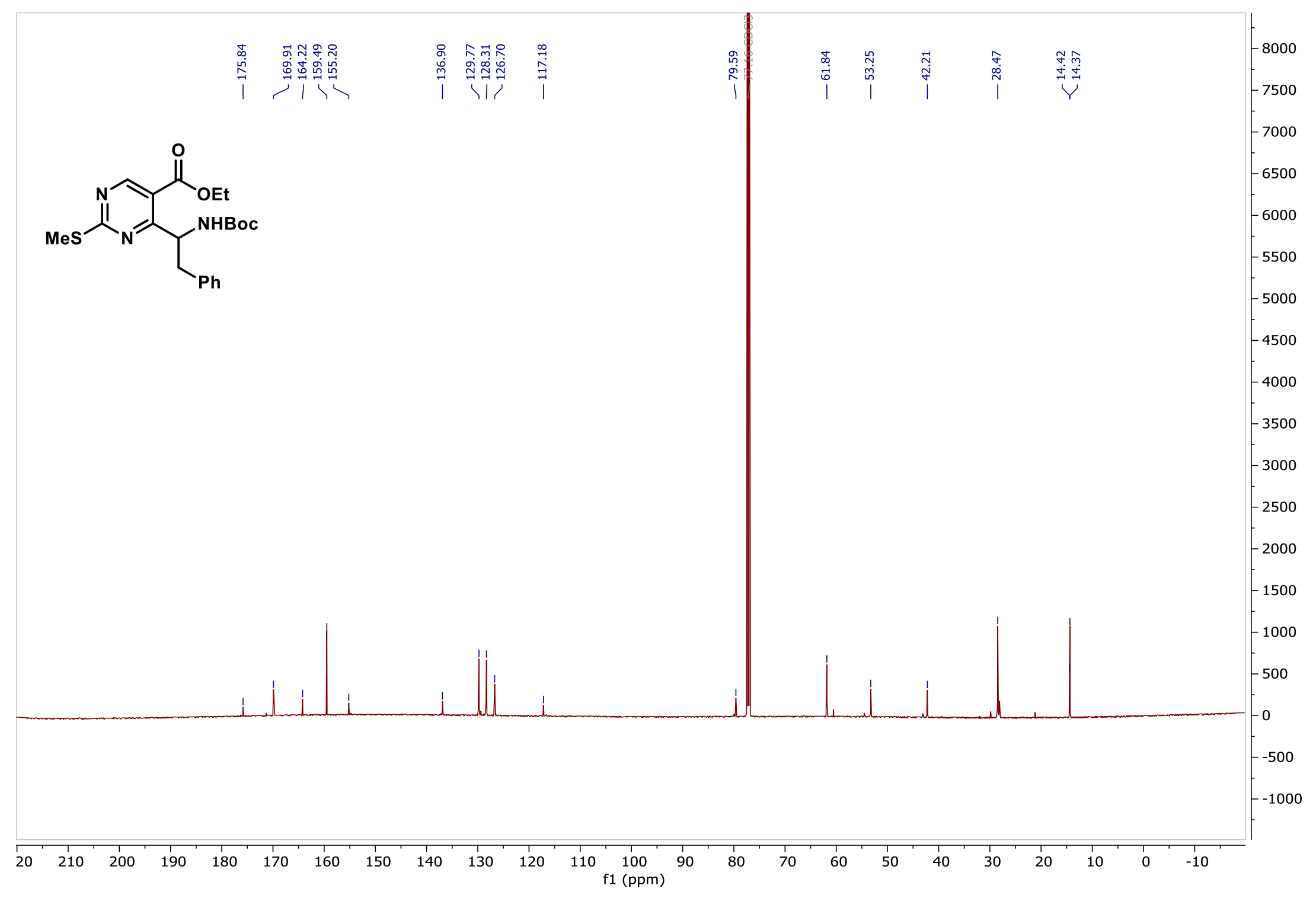


Compound $16,{ }^{1} \mathrm{H}$ NMR $\left(\mathrm{CDCl}_{3}, 600 \mathrm{MHz}\right)$

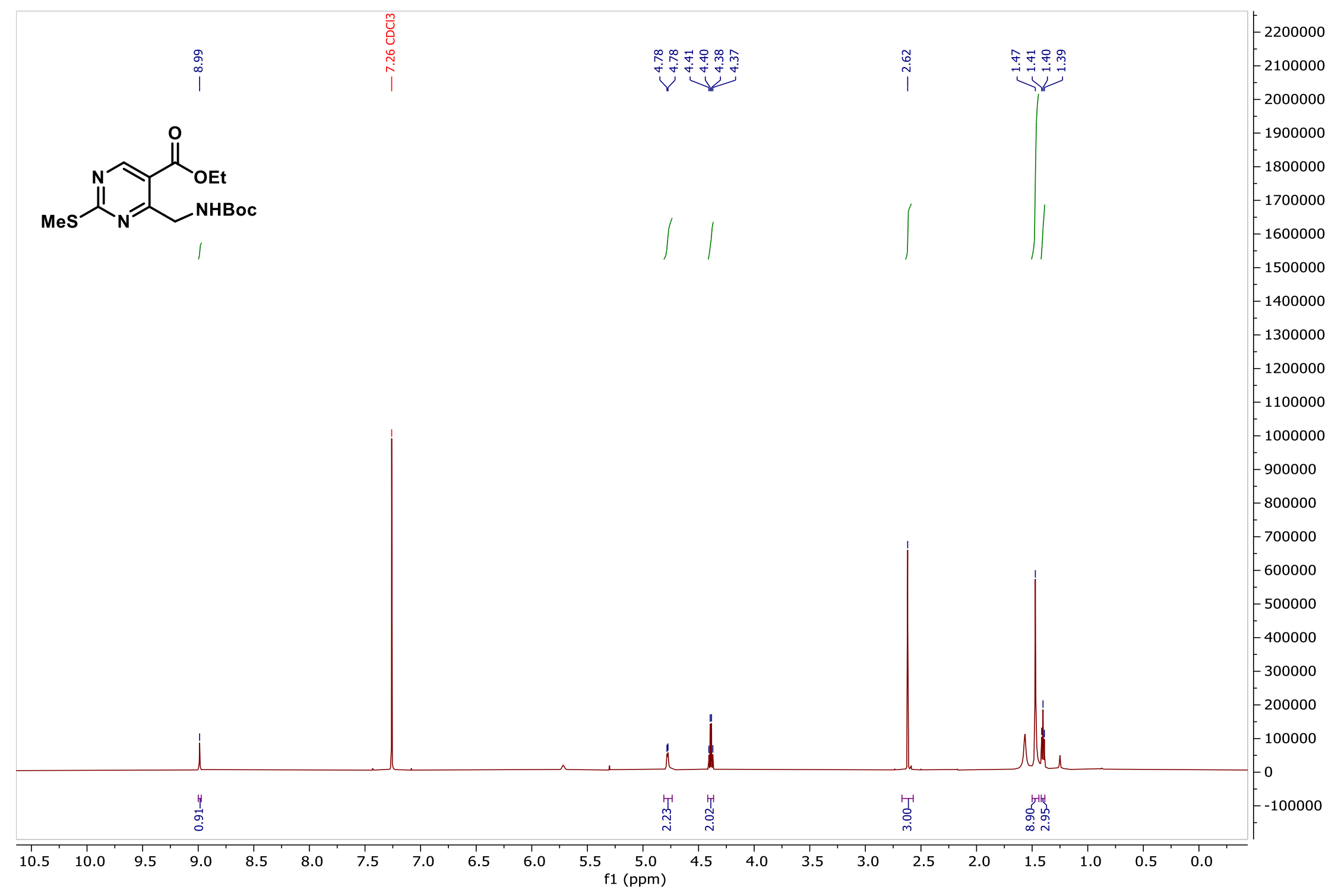


Compound $16,{ }^{13} \mathrm{C}\left\{{ }^{1} \mathrm{H}\right\} \operatorname{NMR}\left(\mathrm{CDCl}_{3}, 151 \mathrm{MHz}\right)$

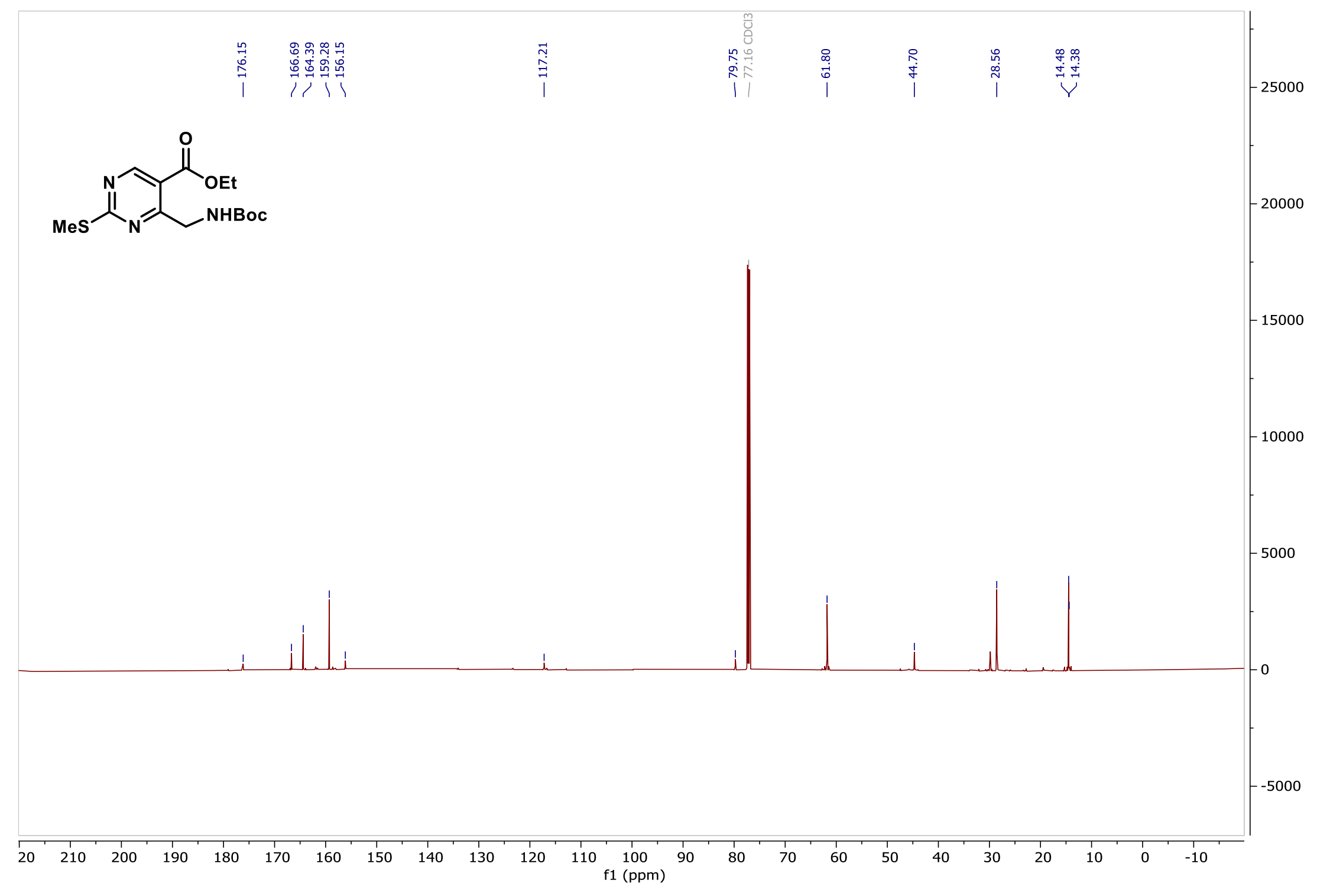


Compound $17,{ }^{1} \mathrm{H}$ NMR $\left(\mathrm{CDCl}_{3}, 600 \mathrm{MHz}\right)$

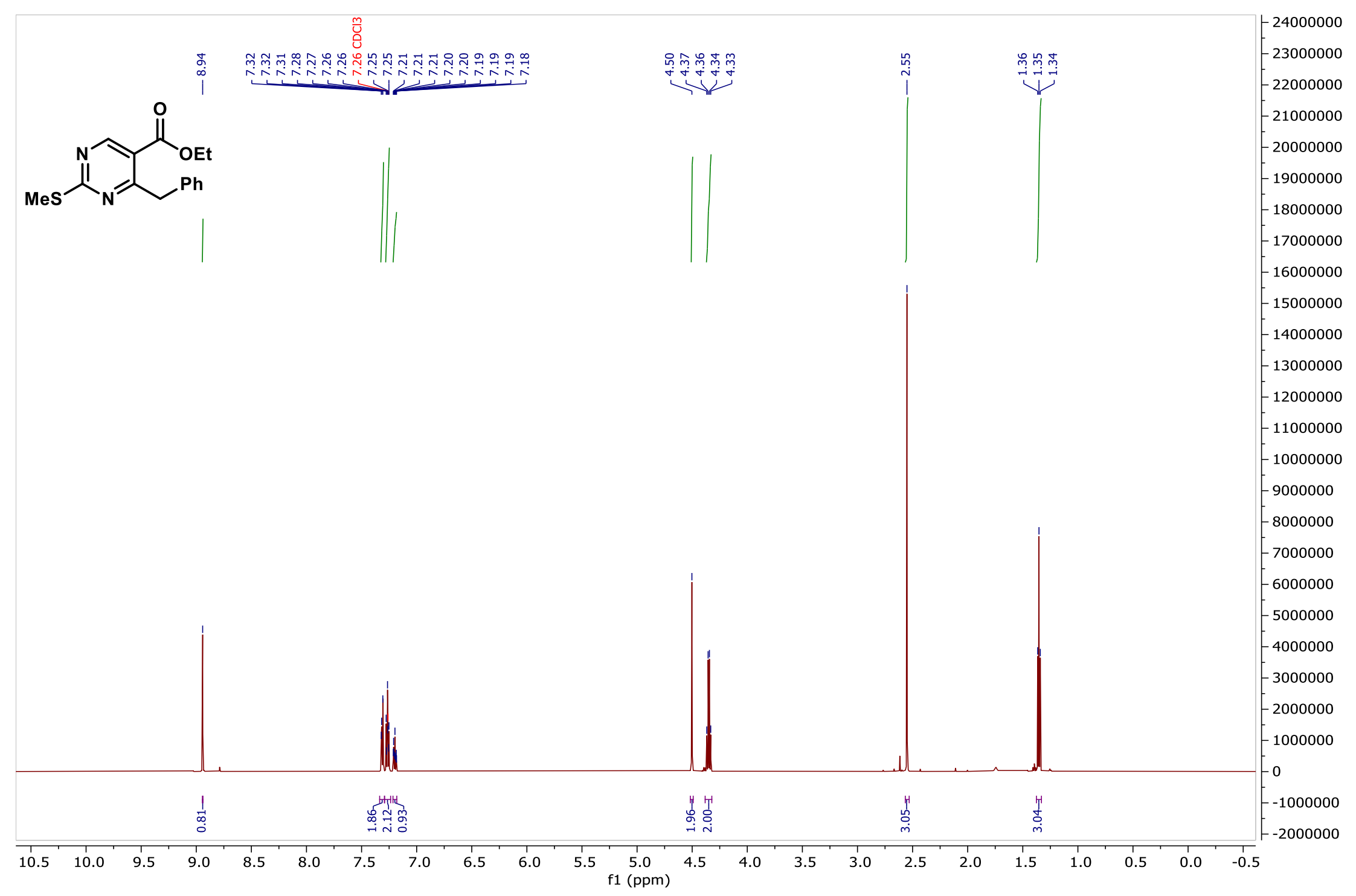


Compound $17,{ }^{13} \mathrm{C}\left\{{ }^{1} \mathrm{H}\right\} \operatorname{NMR}\left(\mathrm{CDCl}_{3}, 151 \mathrm{MHz}\right)$

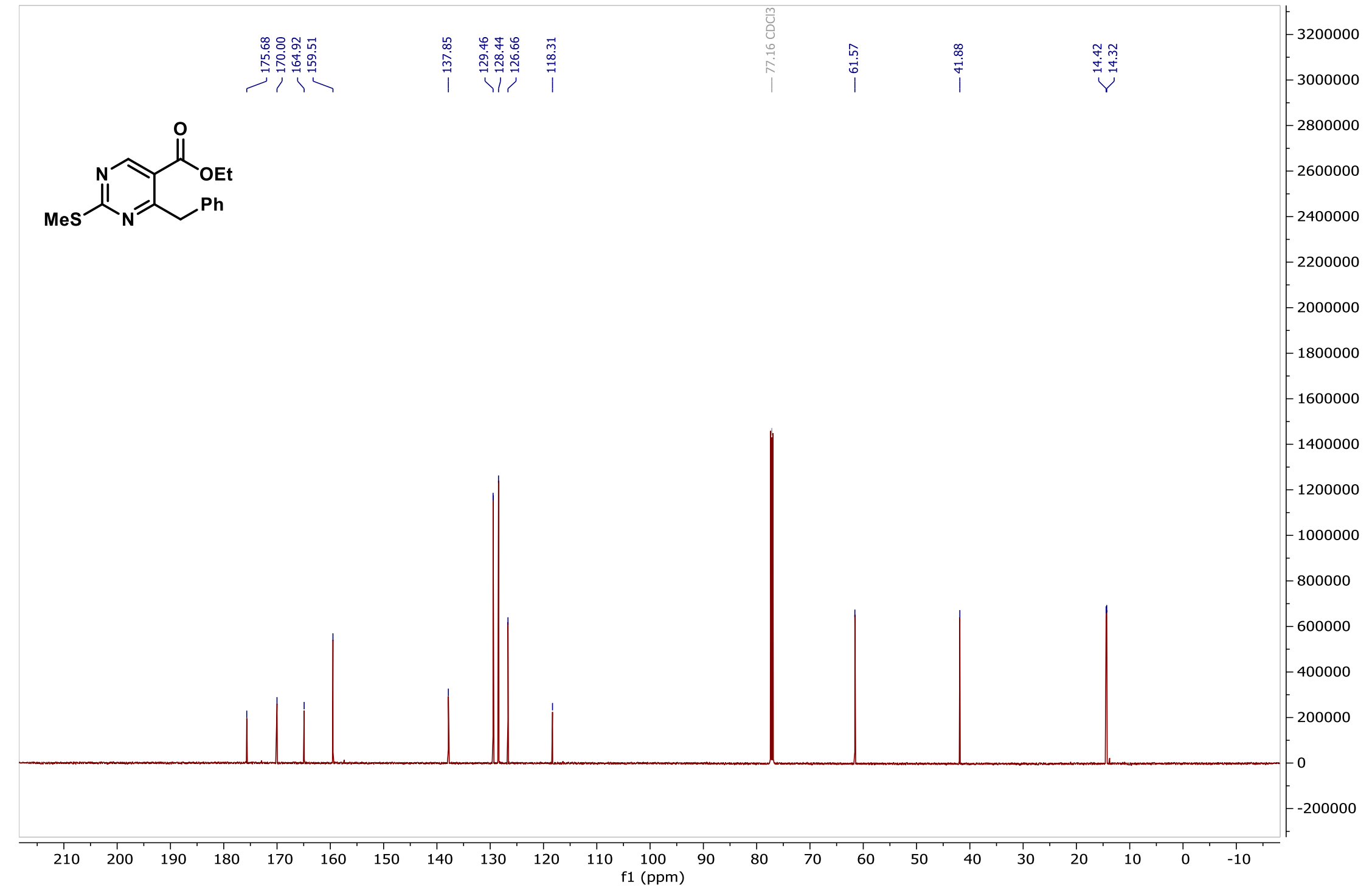


Compound $18,{ }^{1} \mathrm{H}$ NMR $\left(\mathrm{CDCl}_{3}, 600 \mathrm{MHz}\right)$

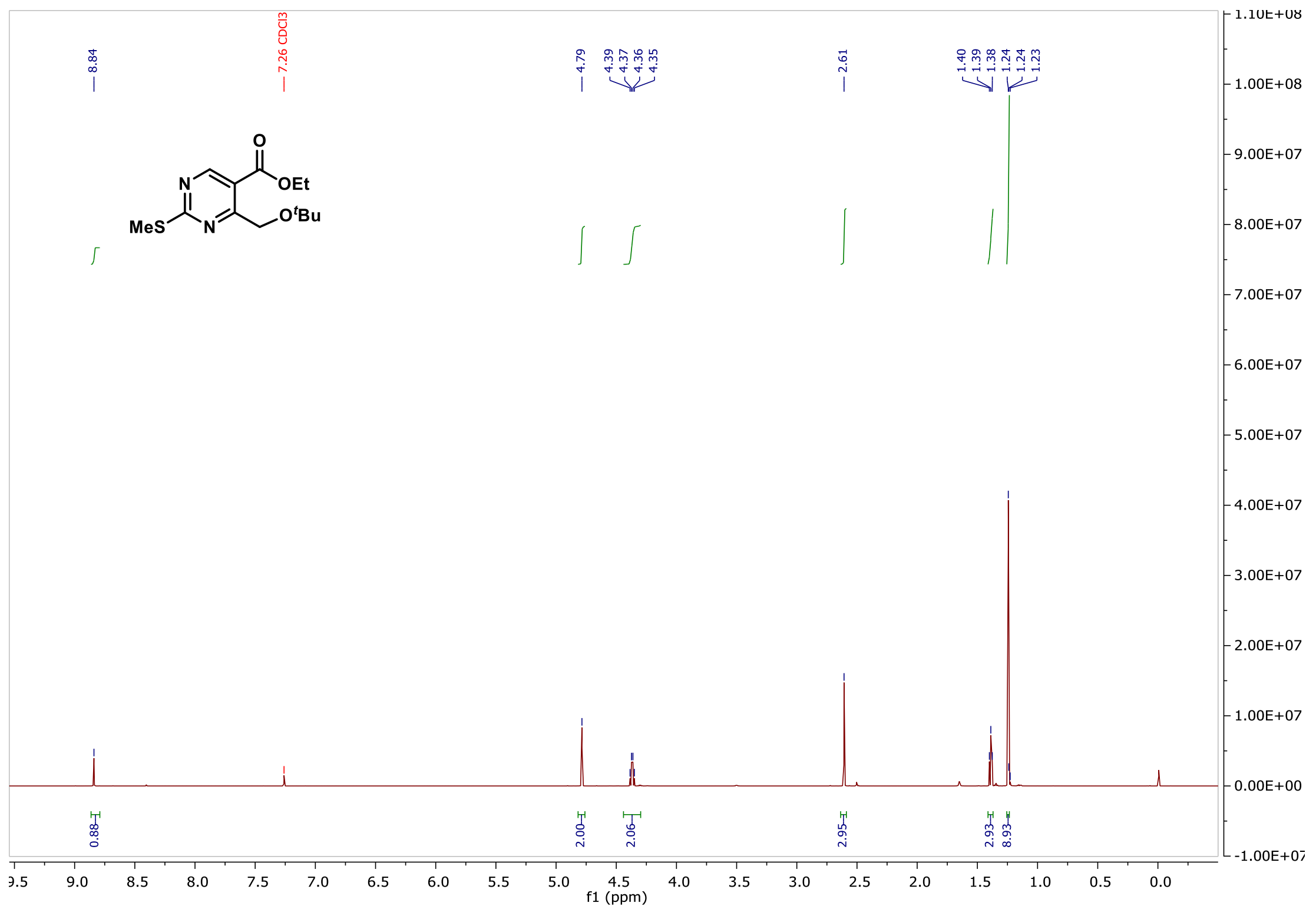


Compound $18,{ }^{13} \mathrm{C}\left\{{ }^{1} \mathrm{H}\right\} \operatorname{NMR}\left(\mathrm{CDCl}_{3}, 151 \mathrm{MHz}\right)$

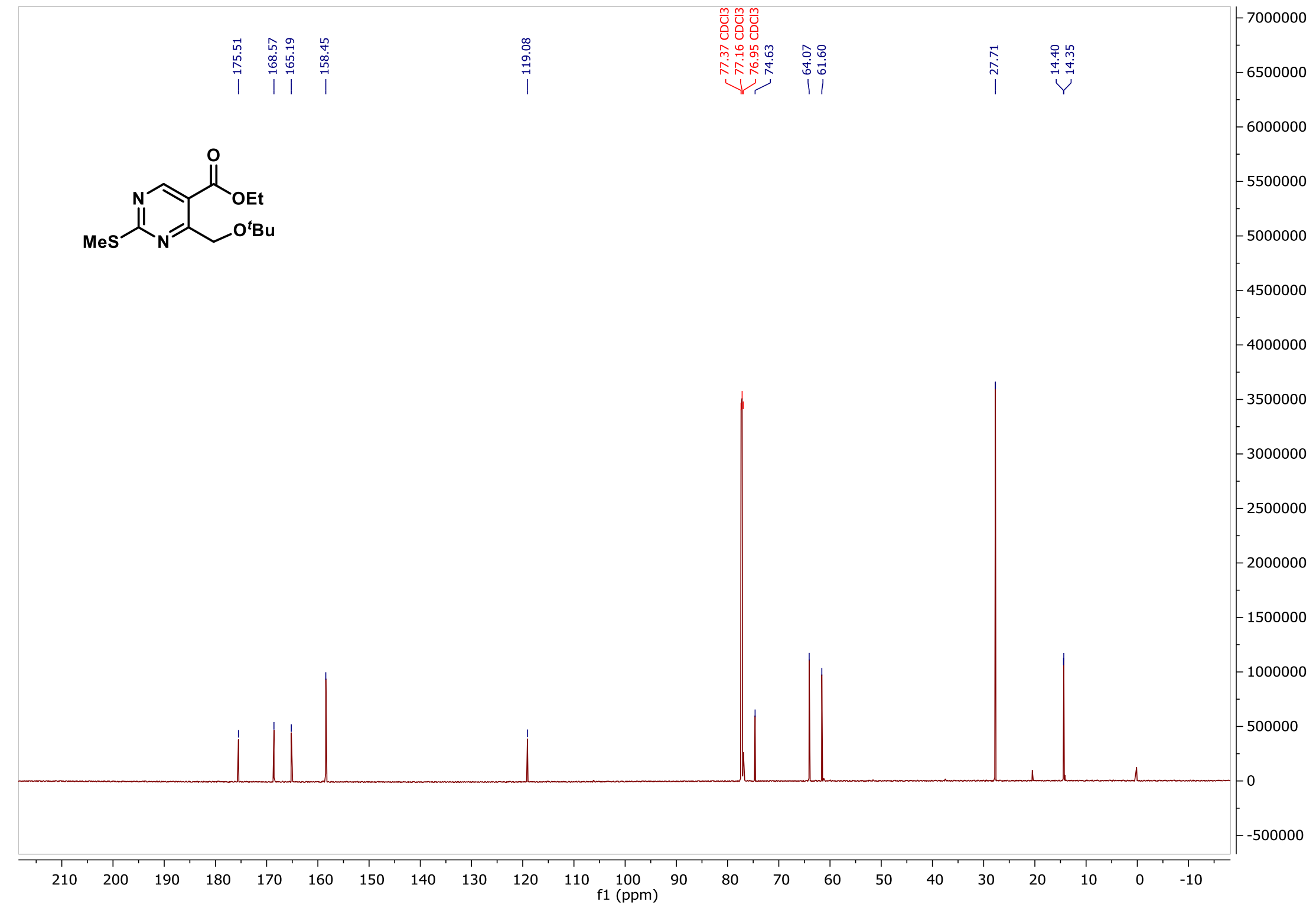


Compound 19, ${ }^{1} \mathrm{H}$ NMR $\left(\mathrm{CDCl}_{3}, 600 \mathrm{MHz}\right)$

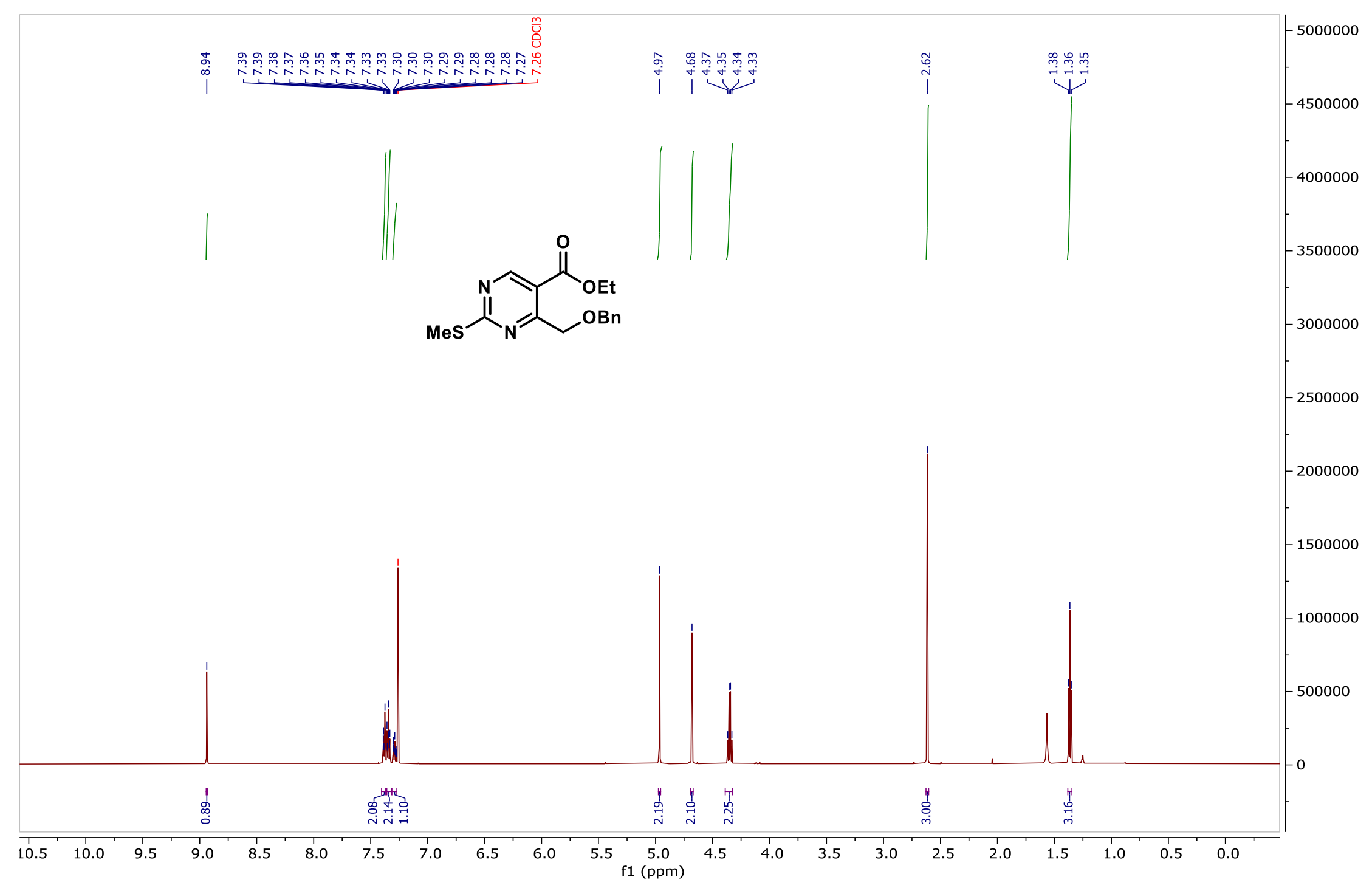


Compound $19,{ }^{13} \mathrm{C}\left\{{ }^{1} \mathrm{H}\right\}$ NMR $\left(\mathrm{CDCl}_{3}, 151 \mathrm{MHz}\right)$

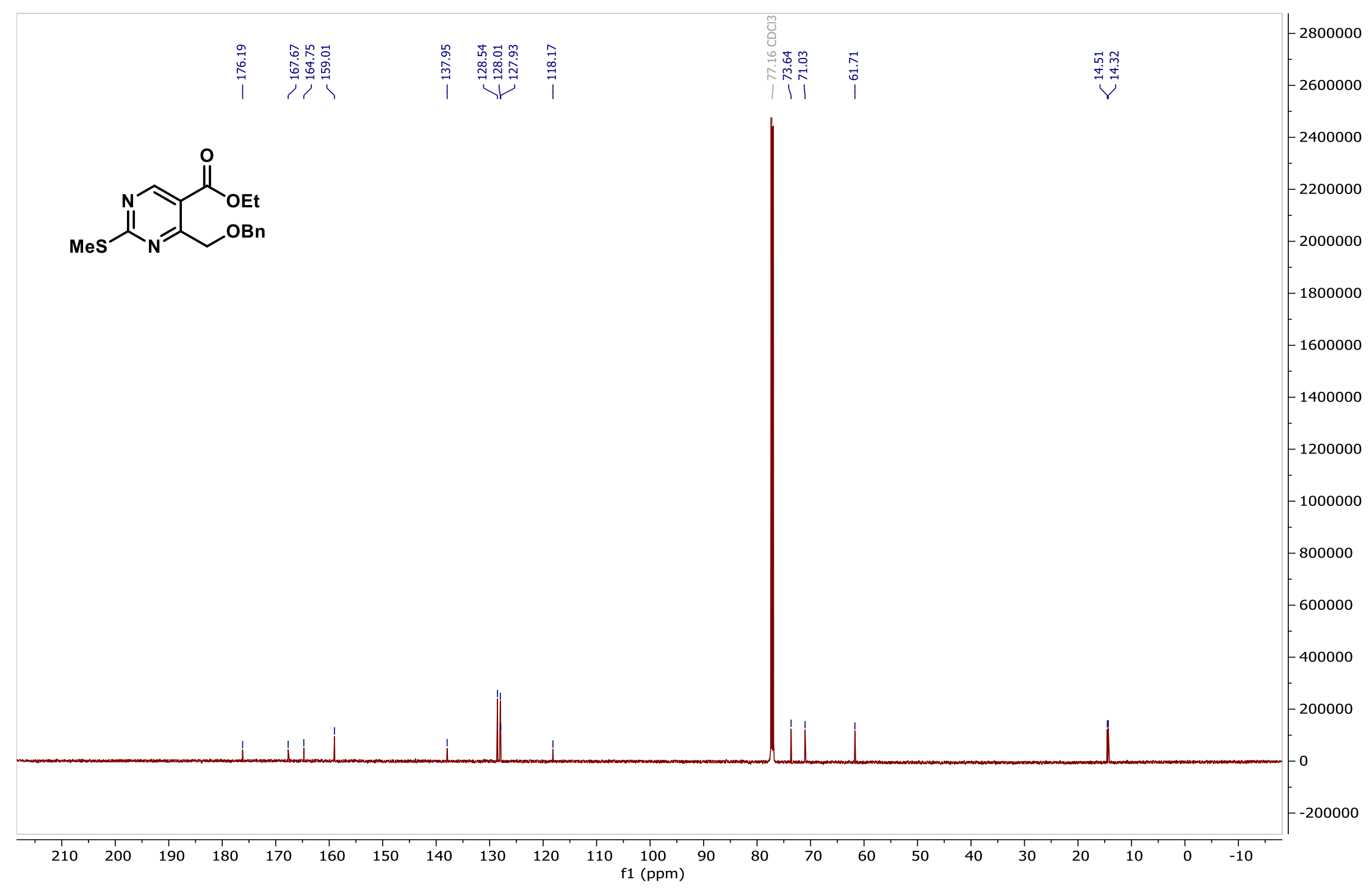


Compound 20, ${ }^{1} \mathrm{H}$ NMR $\left(\mathrm{CDCl}_{3}, 600 \mathrm{MHz}\right)$

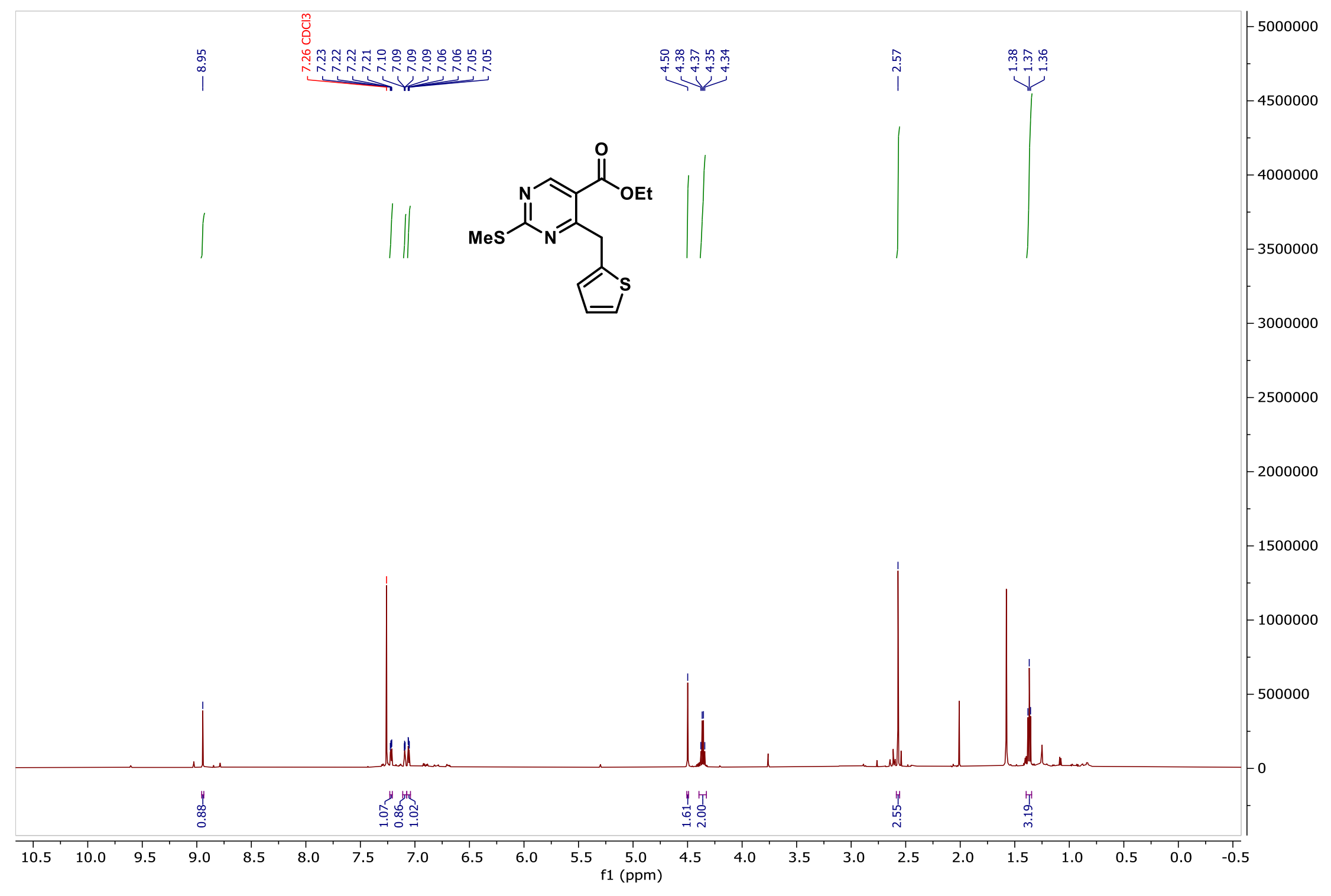


Compound $20,{ }^{13} \mathrm{C}\left\{{ }^{1} \mathrm{H}\right\} \operatorname{NMR}\left(\mathrm{CDCl}_{3}, 151 \mathrm{MHz}\right)$

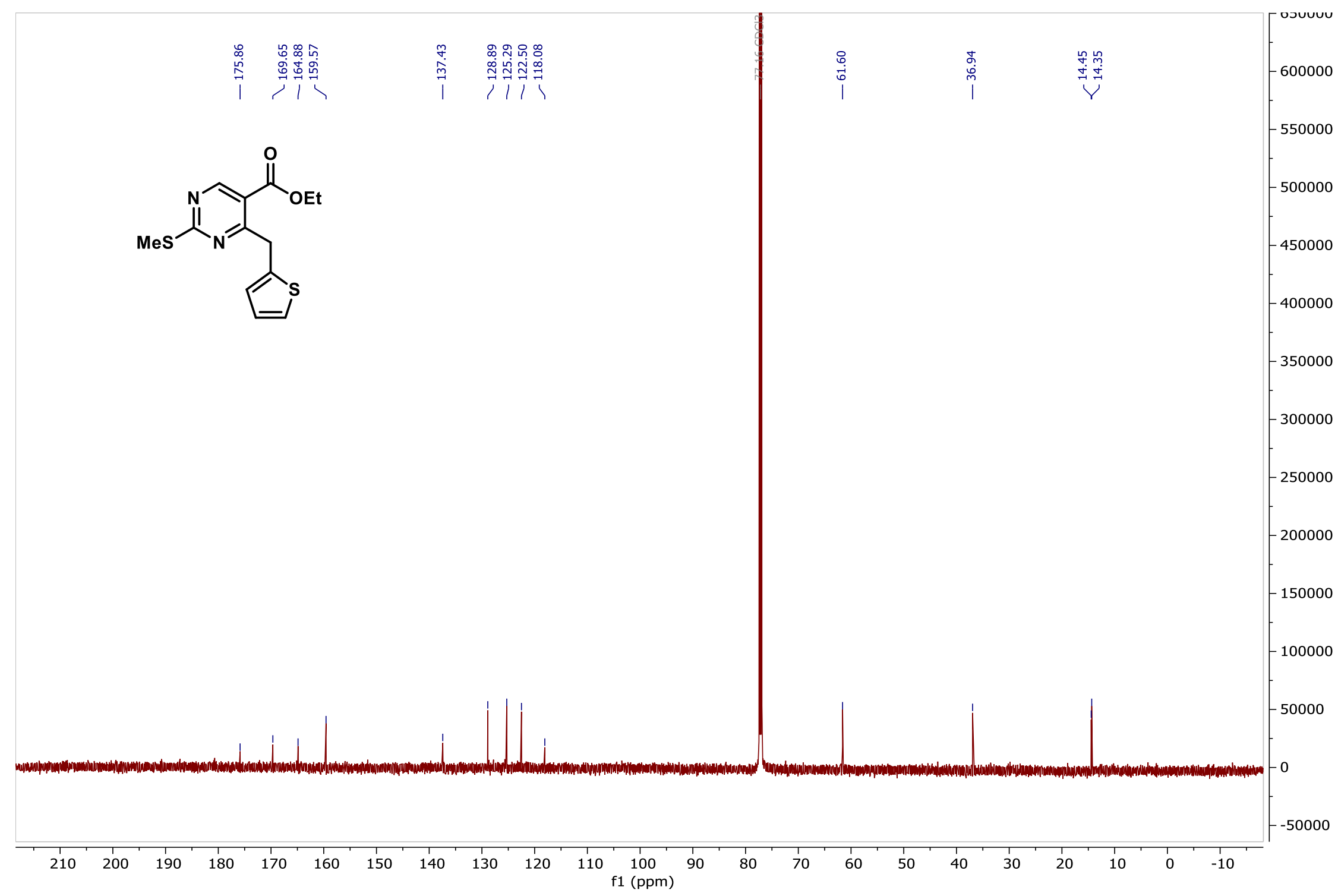


Compound 23, ${ }^{1} \mathrm{H} \mathrm{NMR}\left(\mathrm{CDCl}_{3}, 600 \mathrm{MHz}\right)$

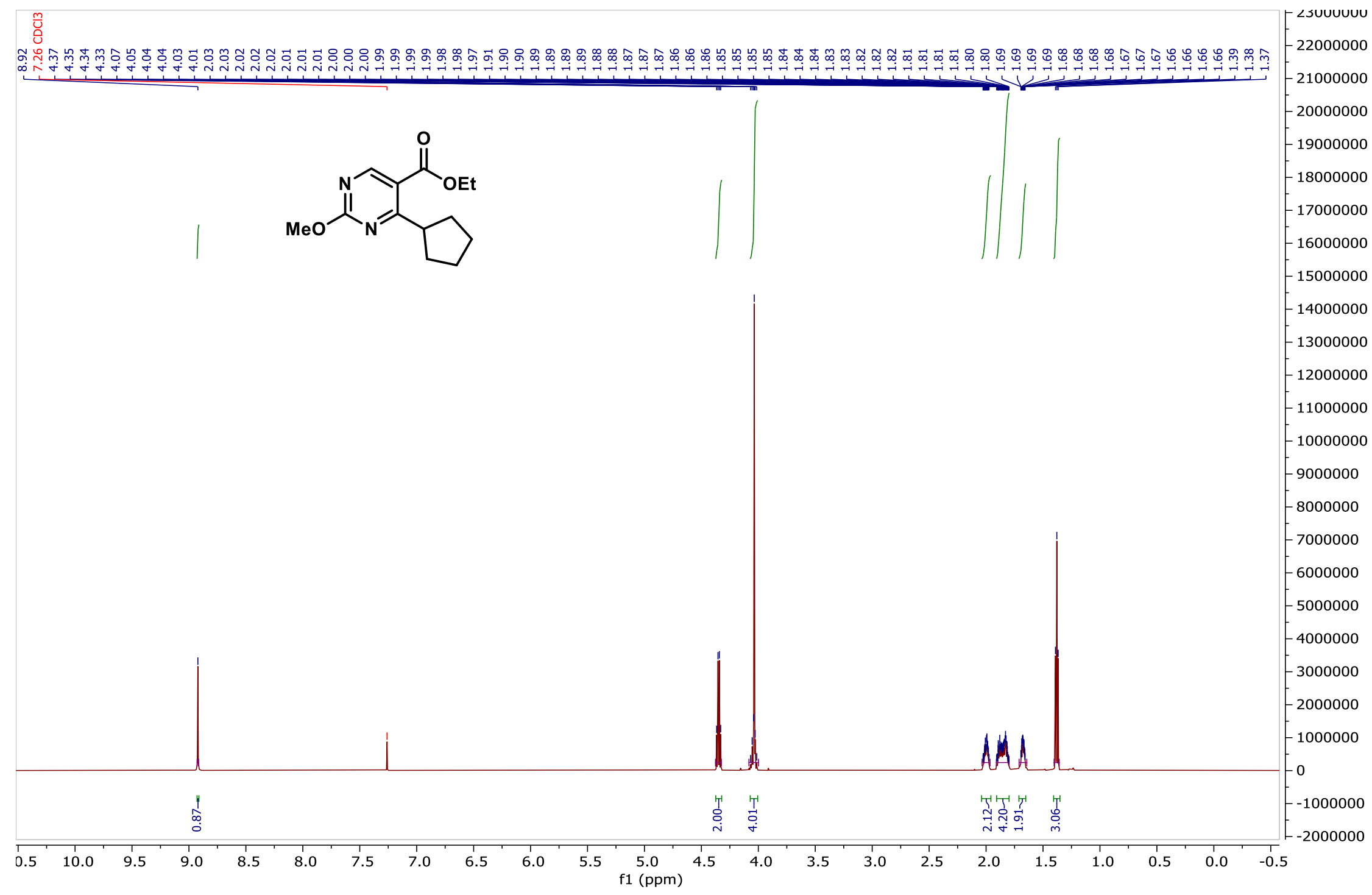


Compound $23,{ }^{13} \mathrm{C}\left\{{ }^{1} \mathrm{H}\right\} \operatorname{NMR}\left(\mathrm{CDCl}_{3}, 151 \mathrm{MHz}\right)$

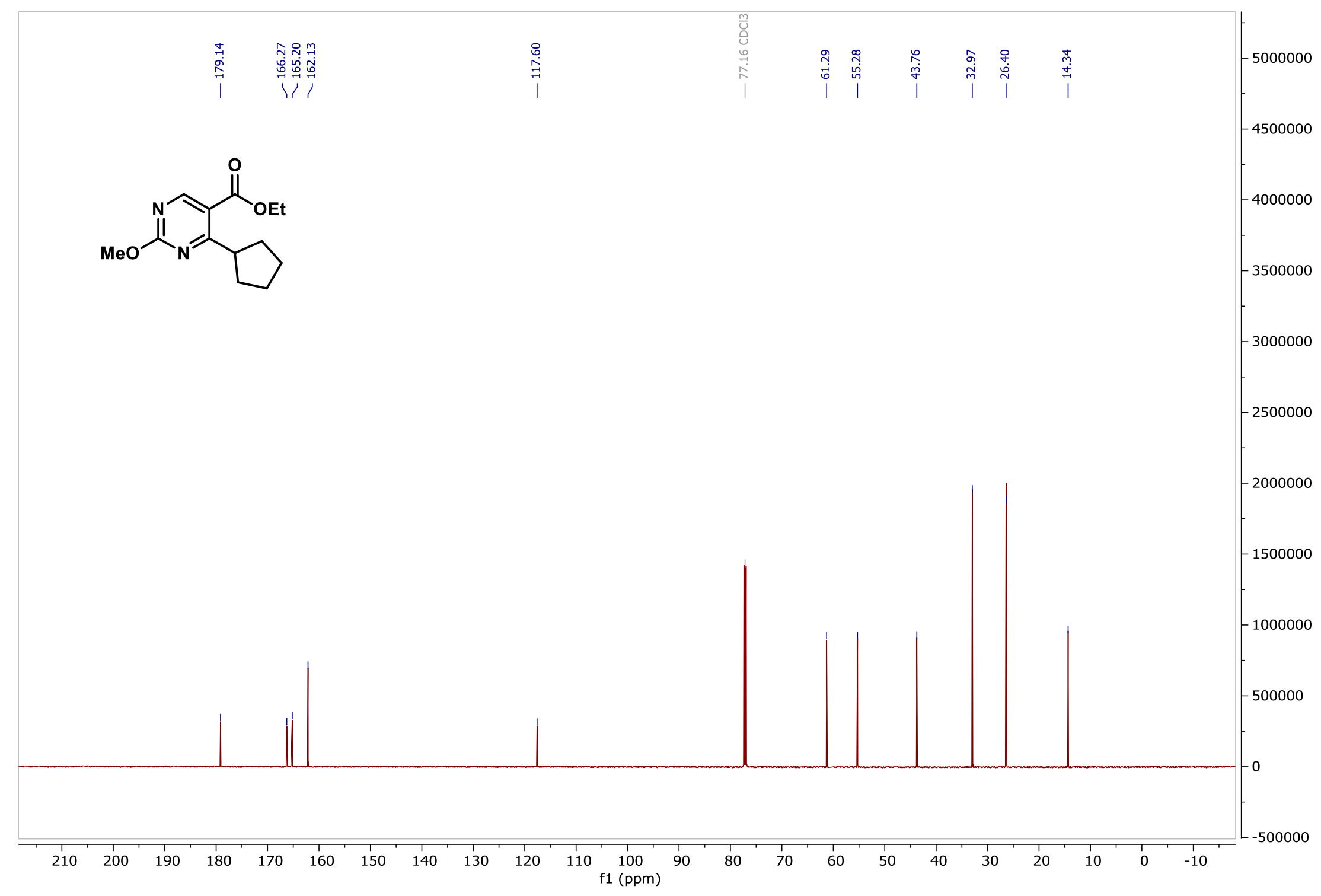


Compound $24,{ }^{1} \mathrm{H}$ NMR $\left(\mathrm{CDCl}_{3}, 600 \mathrm{MHz}\right)$

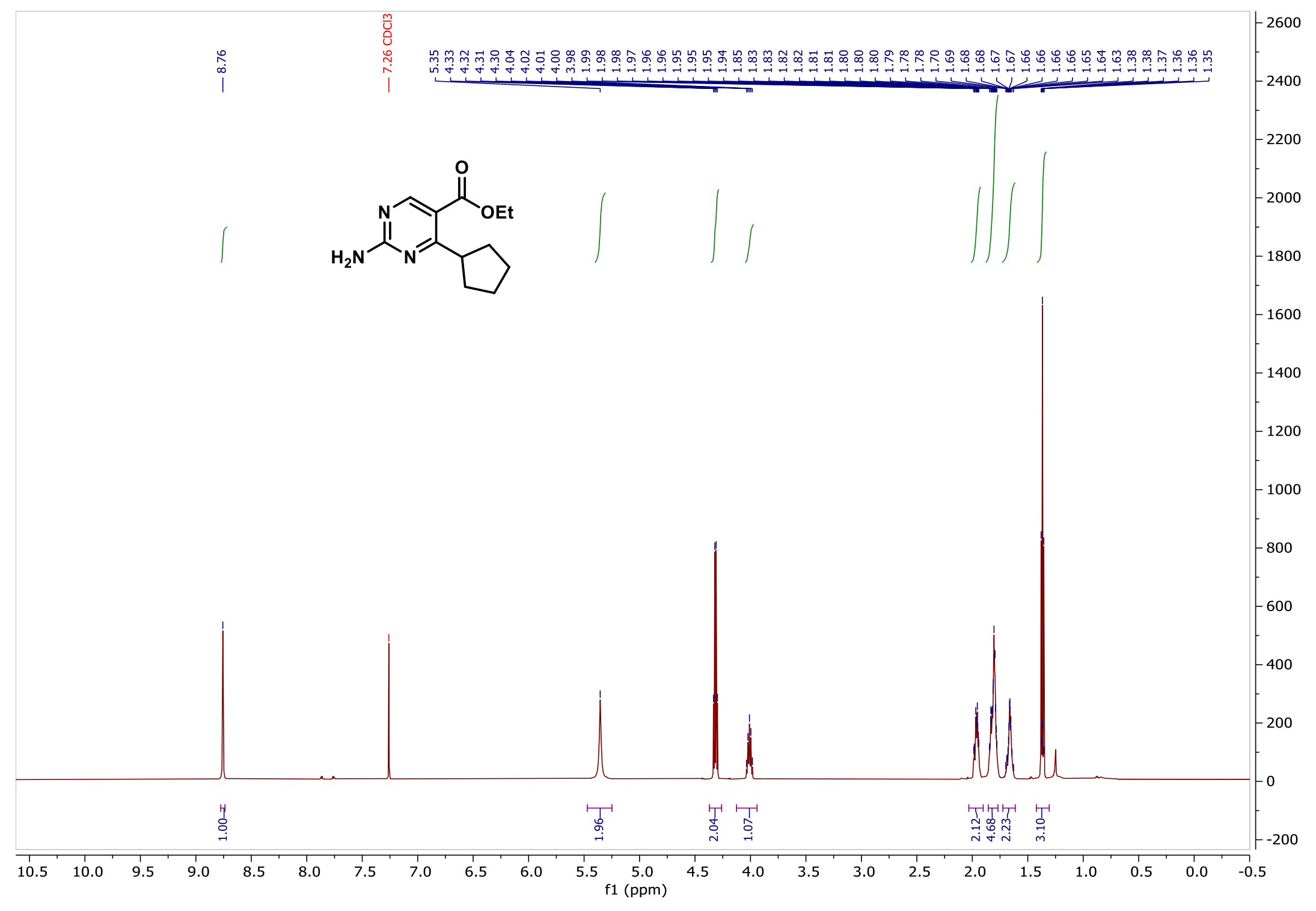


Compound $24,{ }^{13} \mathrm{C}\left\{{ }^{1} \mathrm{H}\right\} \operatorname{NMR}\left(\mathrm{CDCl}_{3}, 151 \mathrm{MHz}\right)$

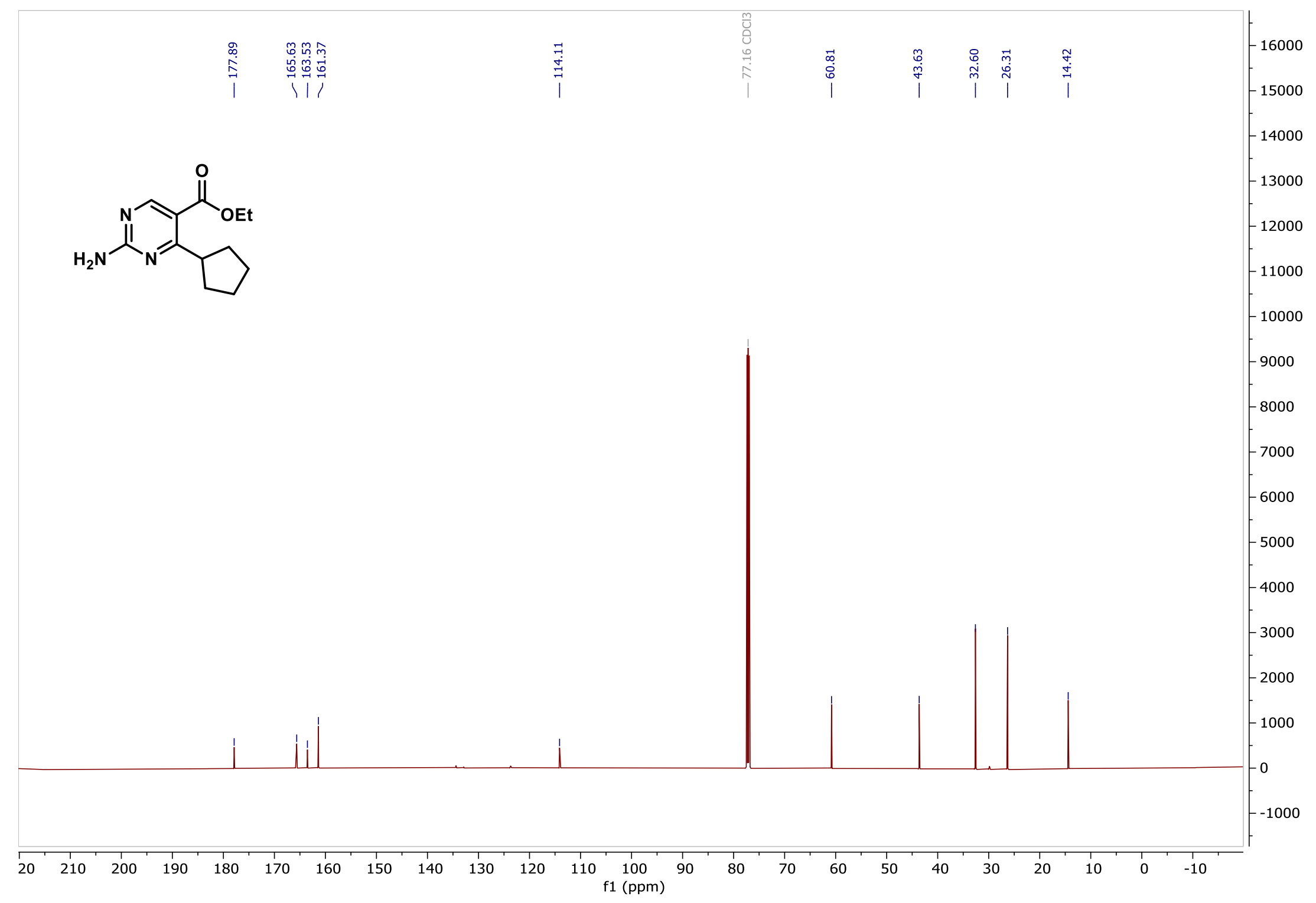


Compound $25,{ }^{1} \mathrm{H}$ NMR $\left(\mathrm{CDCl}_{3}, 600 \mathrm{MHz}\right)$

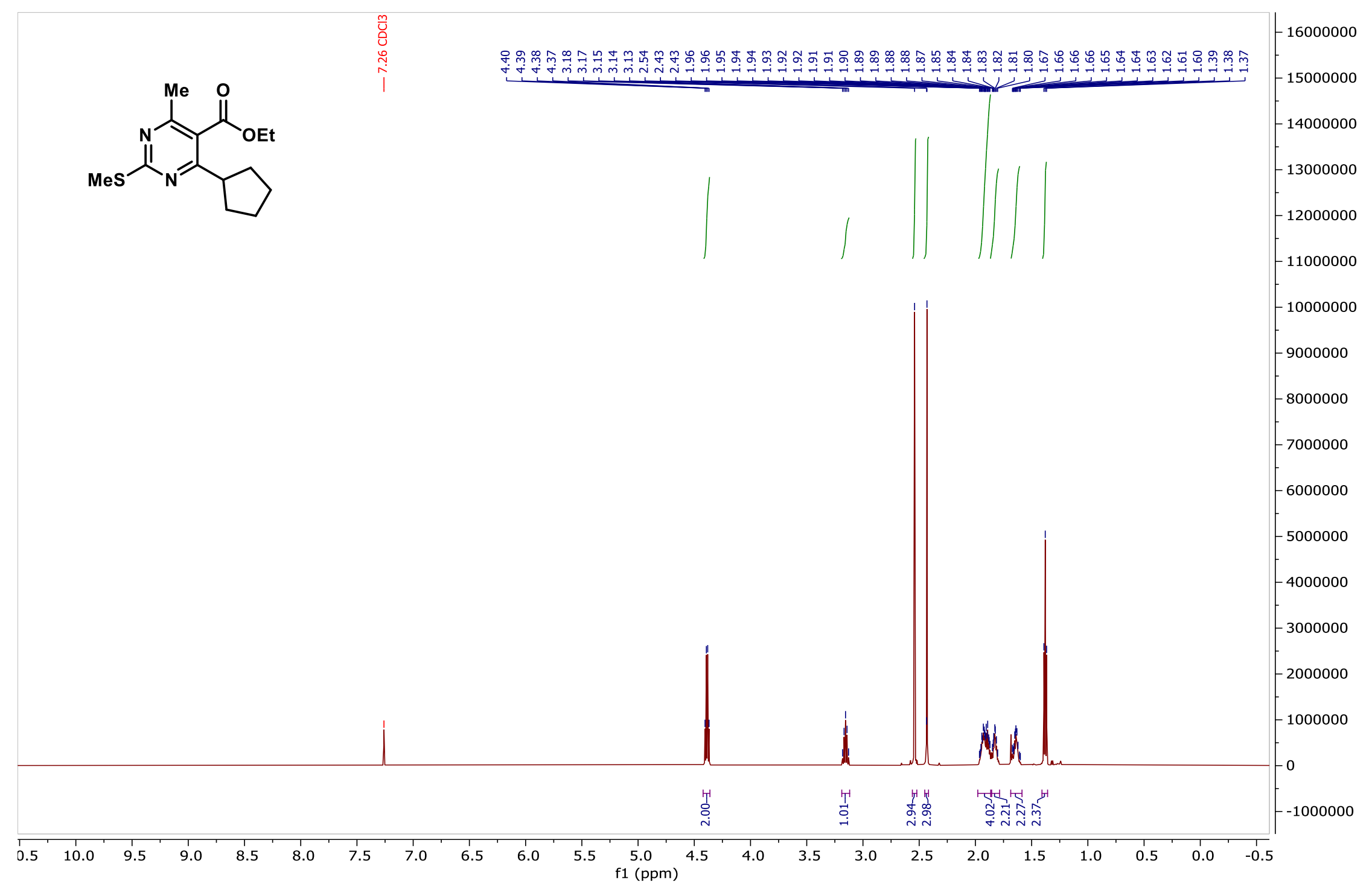


Compound $25,{ }^{13} \mathrm{C}\left\{{ }^{1} \mathrm{H}\right\} \operatorname{NMR}\left(\mathrm{CDCl}_{3}, 151 \mathrm{MHz}\right)$

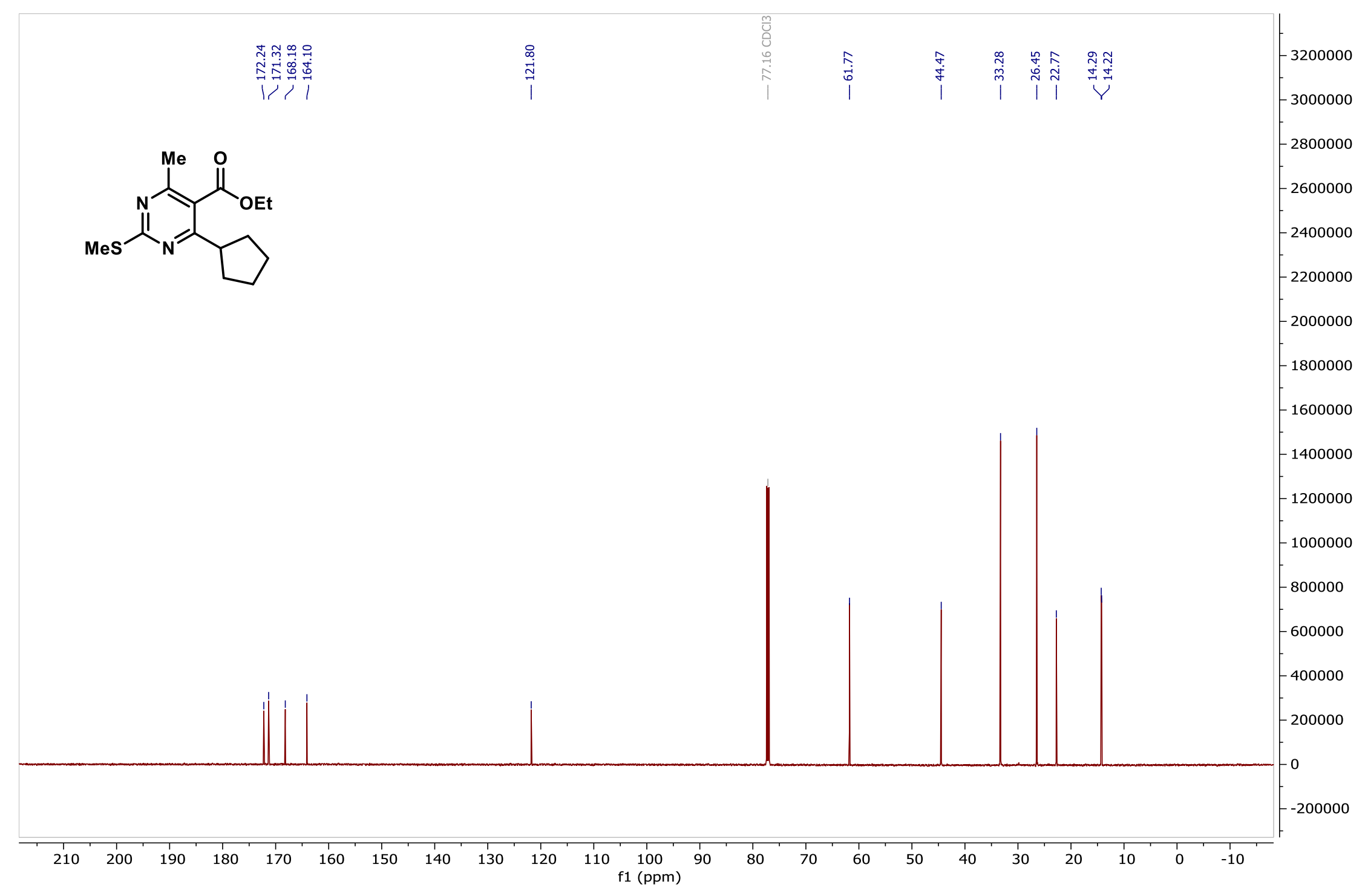


Compound $26,{ }^{1} \mathrm{H} \mathrm{NMR}\left(\mathrm{CDCl}_{3}, 600 \mathrm{MHz}\right)$

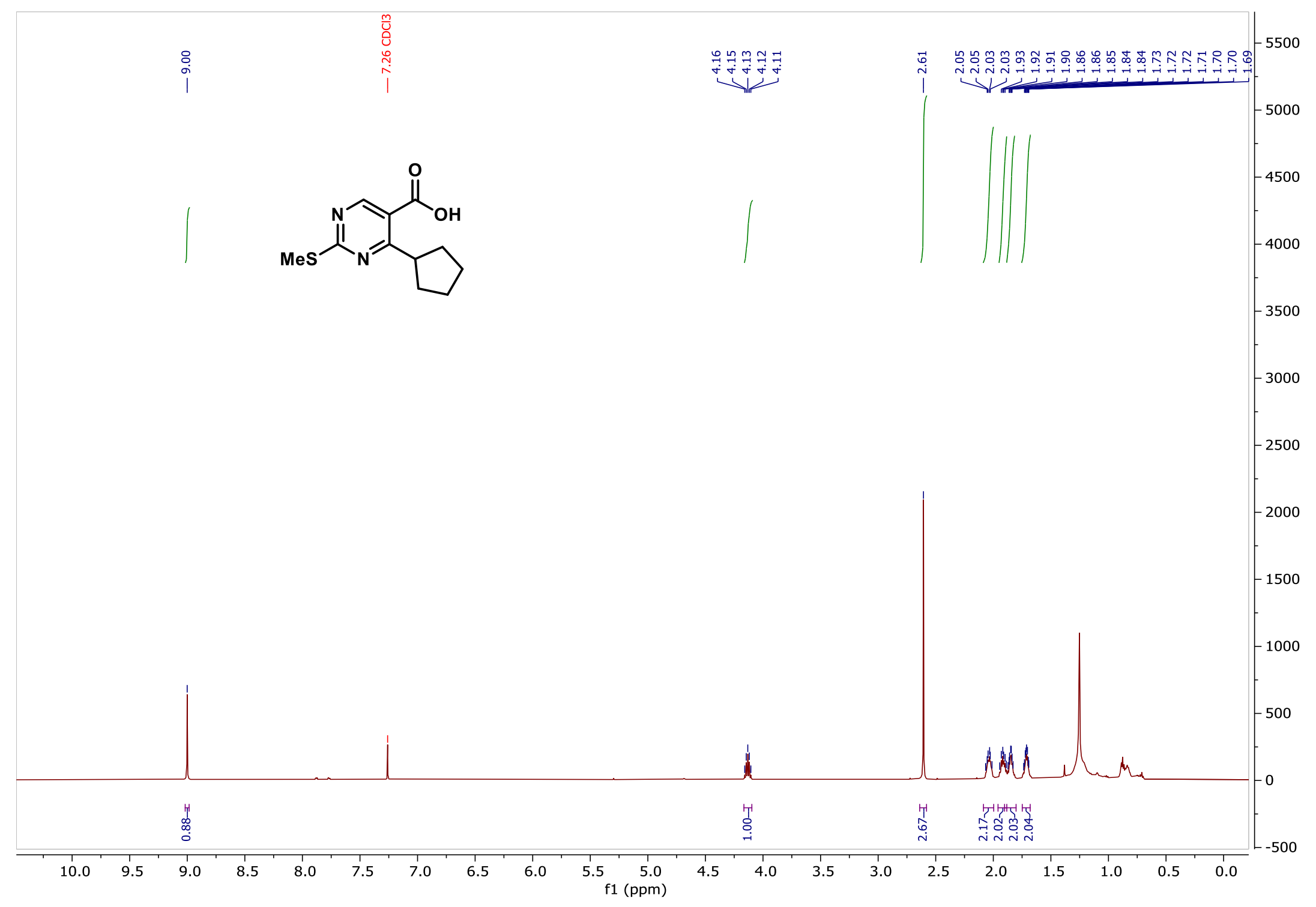


Compound $26,{ }^{13} \mathrm{C}\left\{{ }^{1} \mathrm{H}\right\}$ NMR $\left(\mathrm{CDCl}_{3}, 151 \mathrm{MHz}\right)$

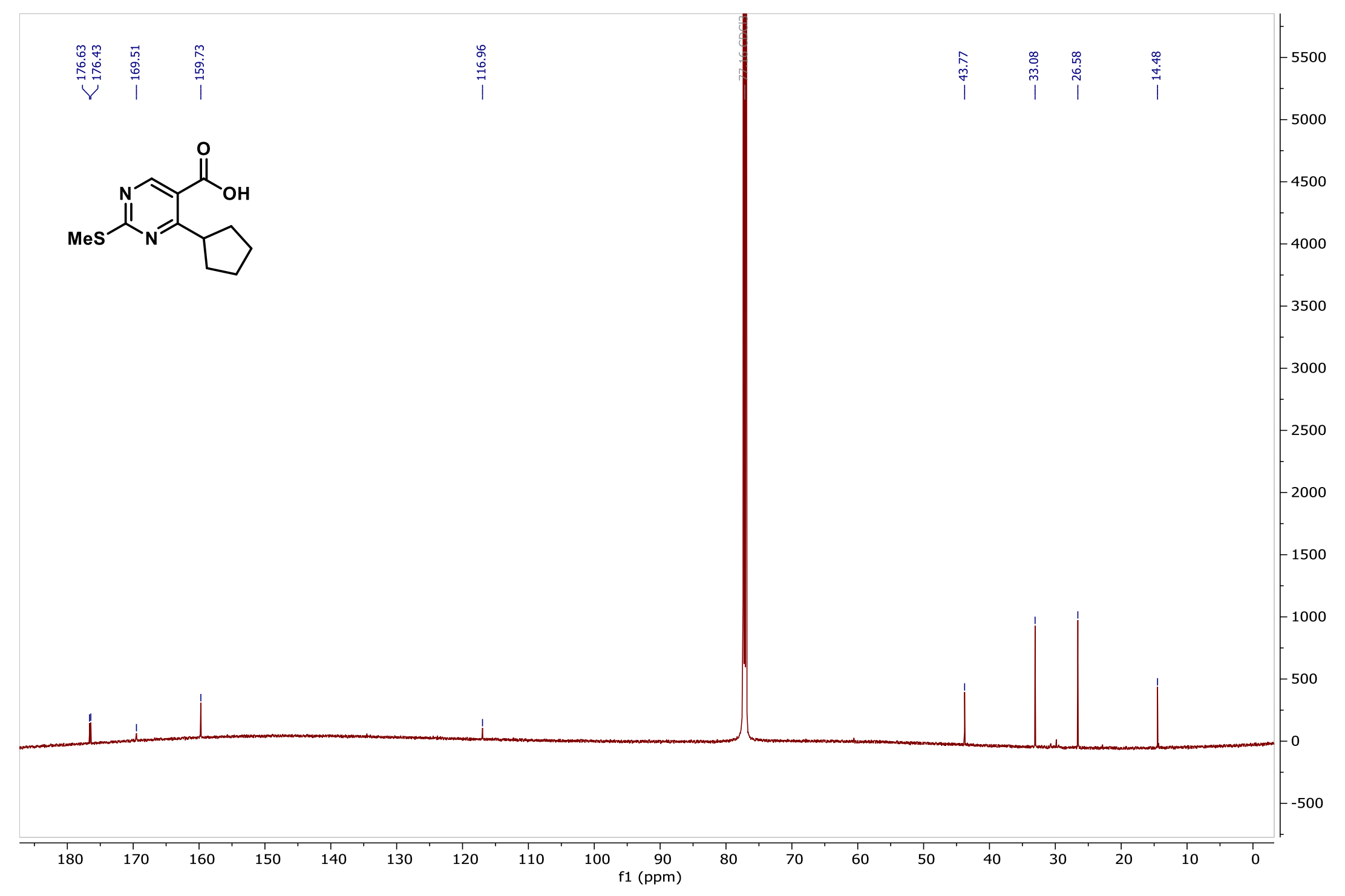


Compound $27,{ }^{1} \mathrm{H}$ NMR $\left(\mathrm{CDCl}_{3}, 600 \mathrm{MHz}\right)$

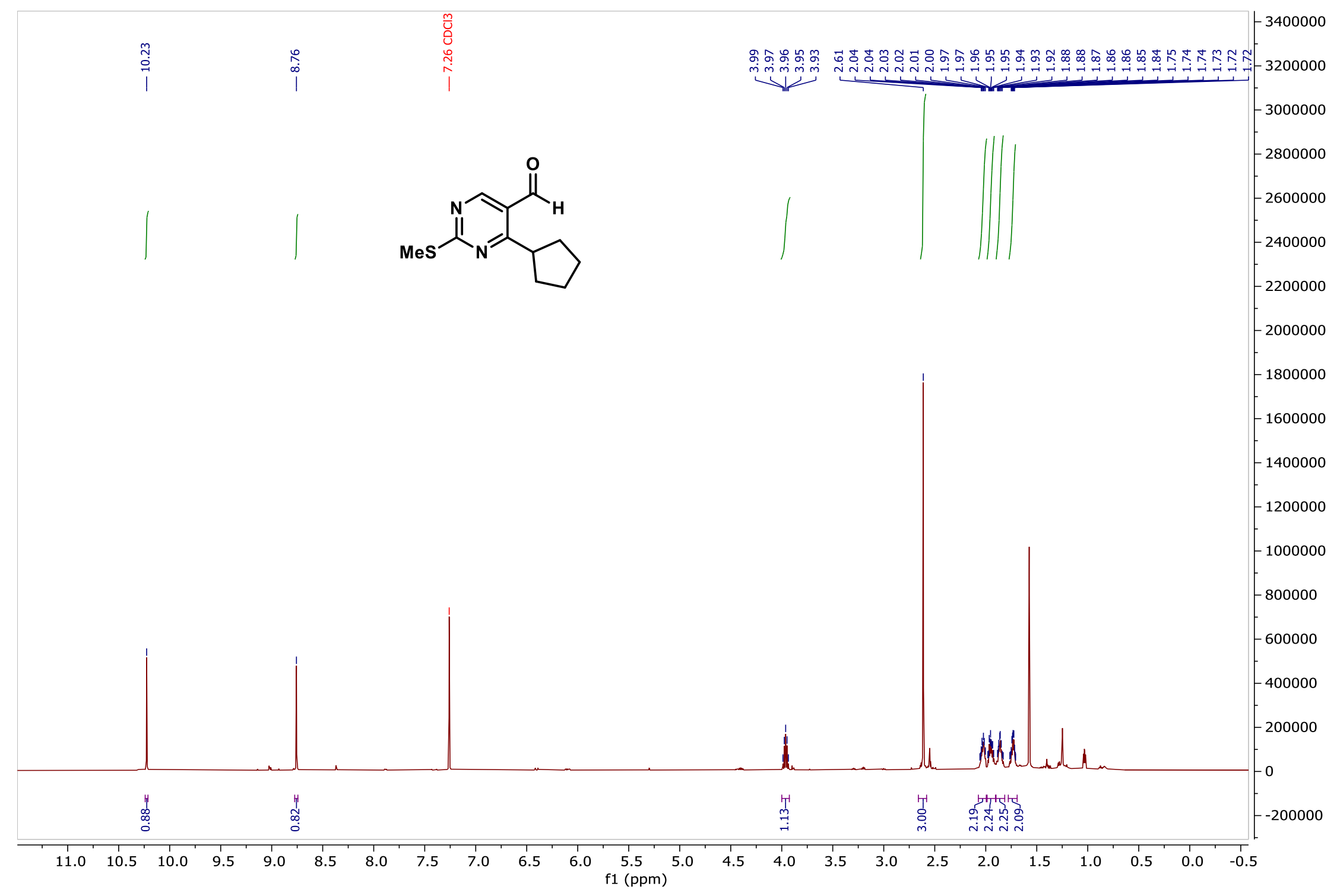


Compound $27,{ }^{13} \mathrm{C}\left\{{ }^{1} \mathrm{H}\right\} \operatorname{NMR}\left(\mathrm{CDCl}_{3}, 151 \mathrm{MHz}\right)$

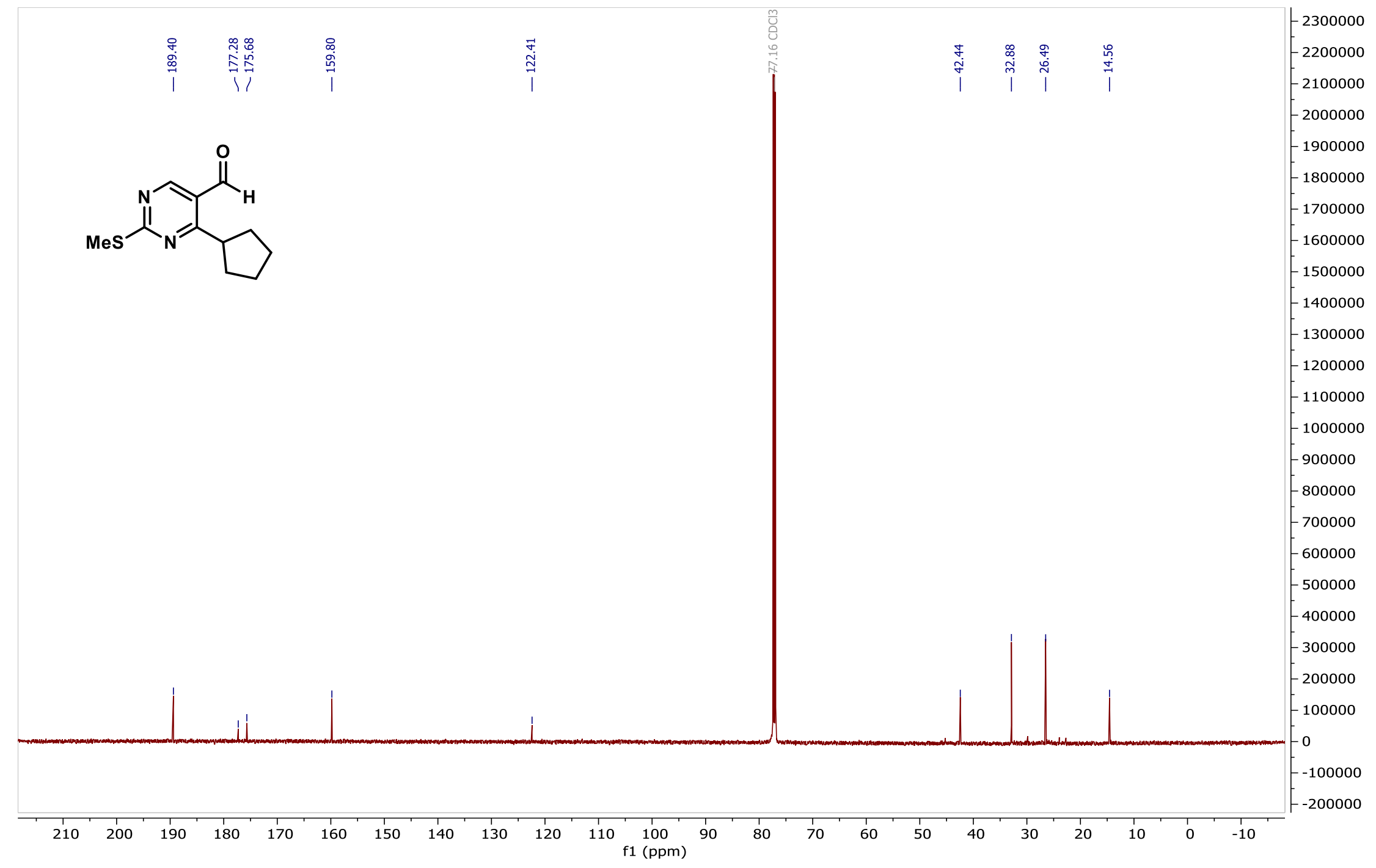


Compound $28,{ }^{1} \mathrm{H}$ NMR $\left(\mathrm{CDCl}_{3}, 600 \mathrm{MHz}\right)$

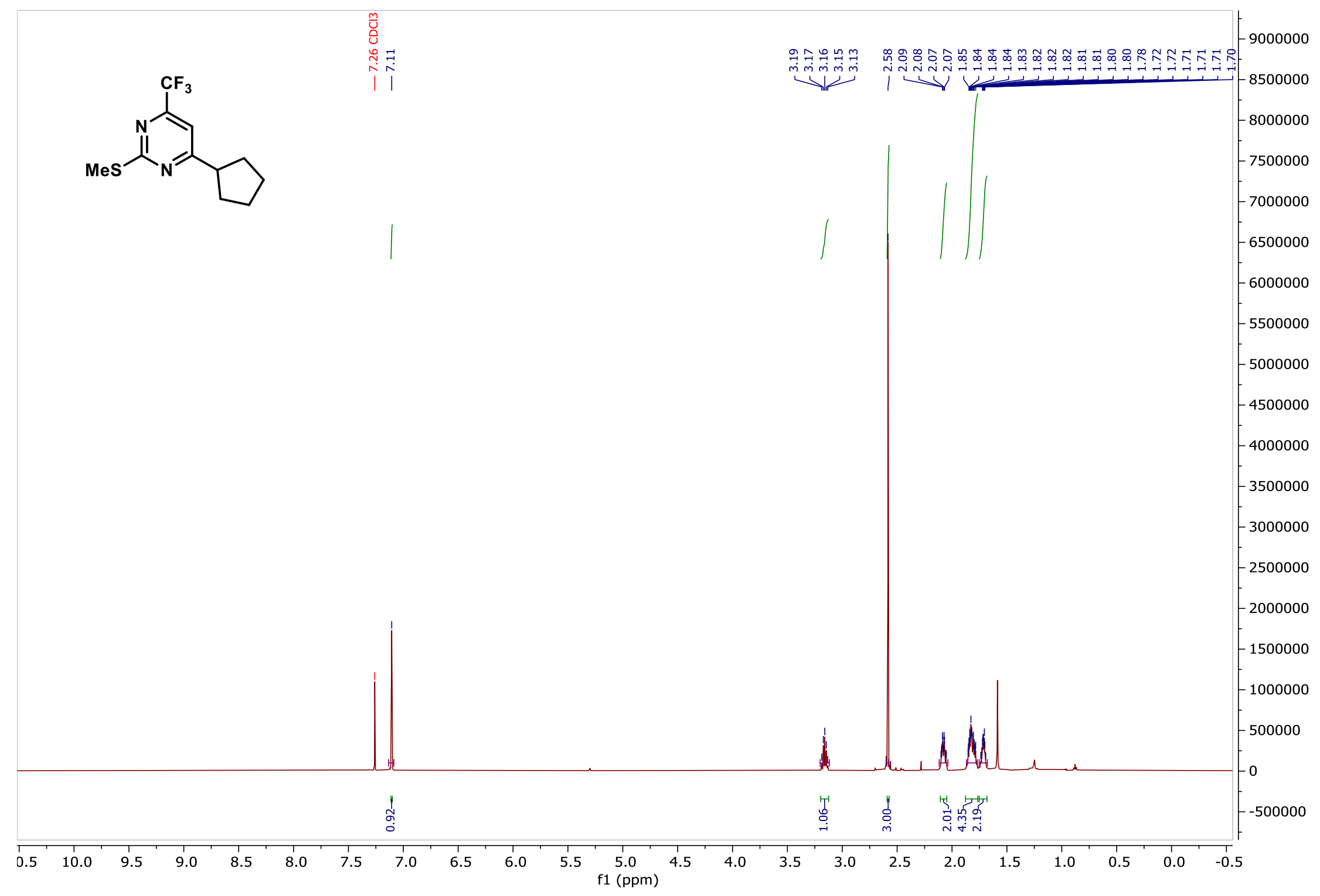


Compound $28,{ }^{13} \mathrm{C}\left\{{ }^{1} \mathrm{H}\right\} \operatorname{NMR}\left(\mathrm{CDCl}_{3}, 151 \mathrm{MHz}\right)$

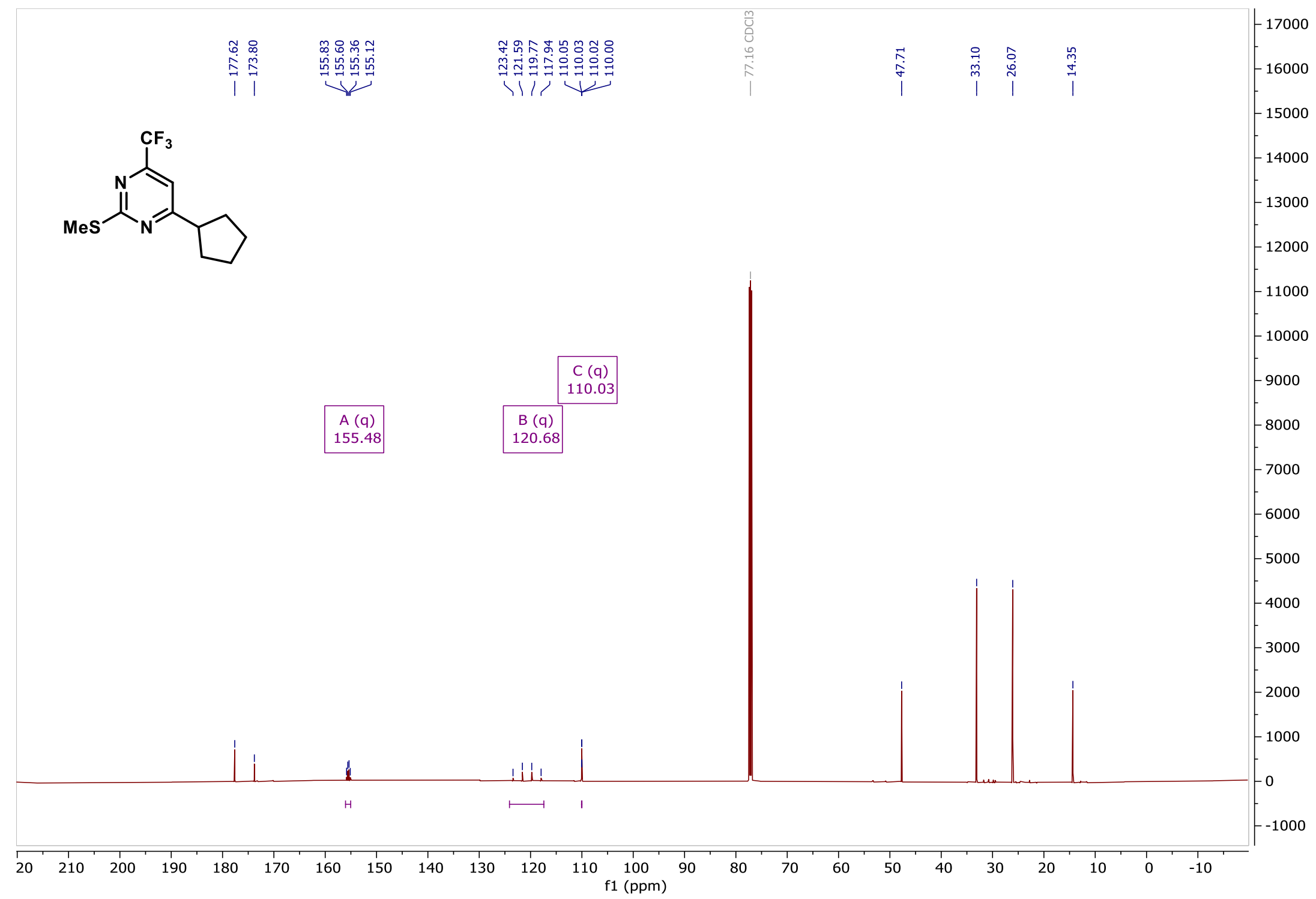




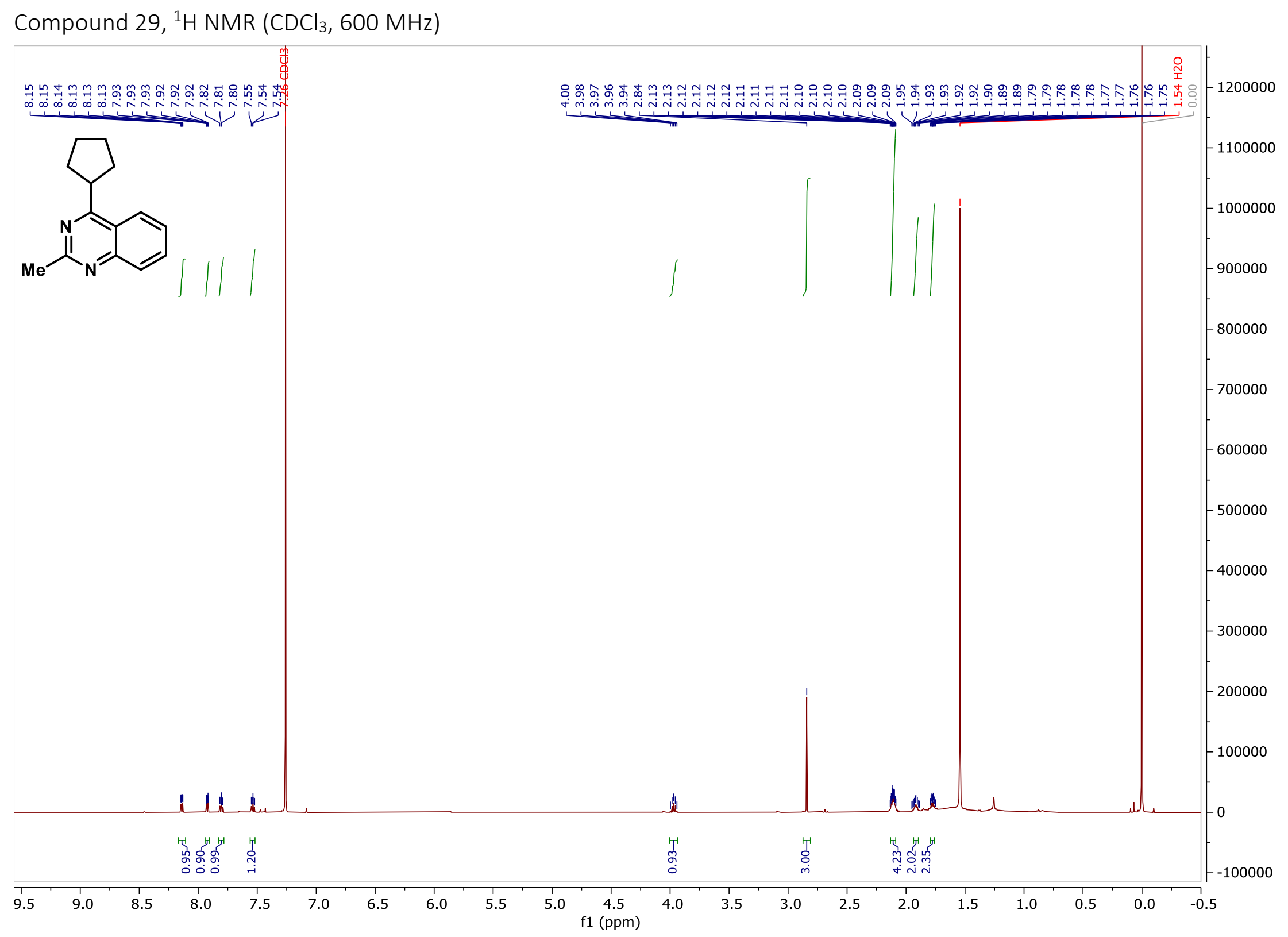


Compound $29,{ }^{13} \mathrm{C}\left\{{ }^{1} \mathrm{H}\right\} \operatorname{NMR}\left(\mathrm{CDCl}_{3}, 151 \mathrm{MHz}\right)$

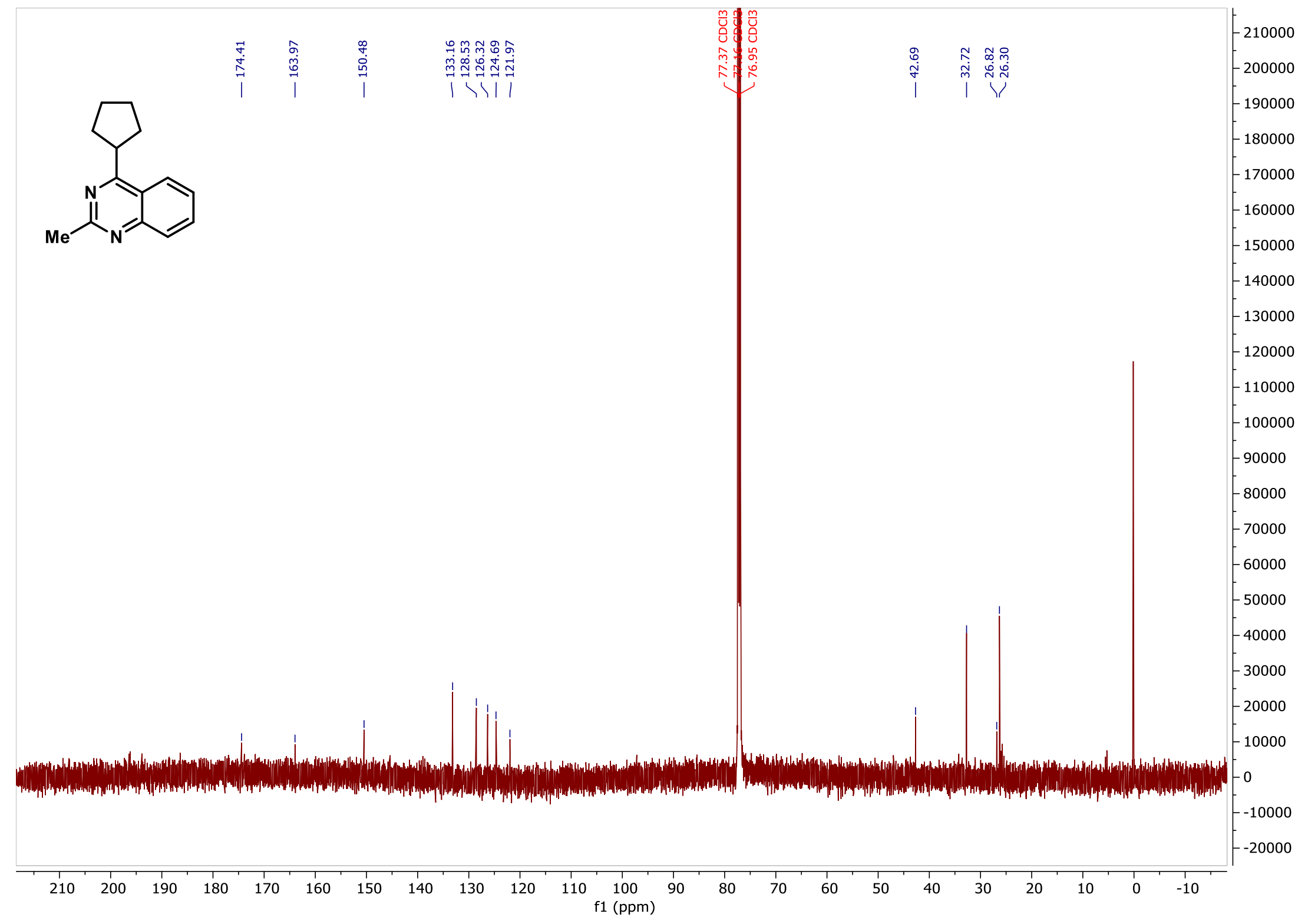


Compound $31,{ }^{1} \mathrm{H} \mathrm{NMR}\left(\mathrm{CDCl}_{3}, 600 \mathrm{MHz}\right)$

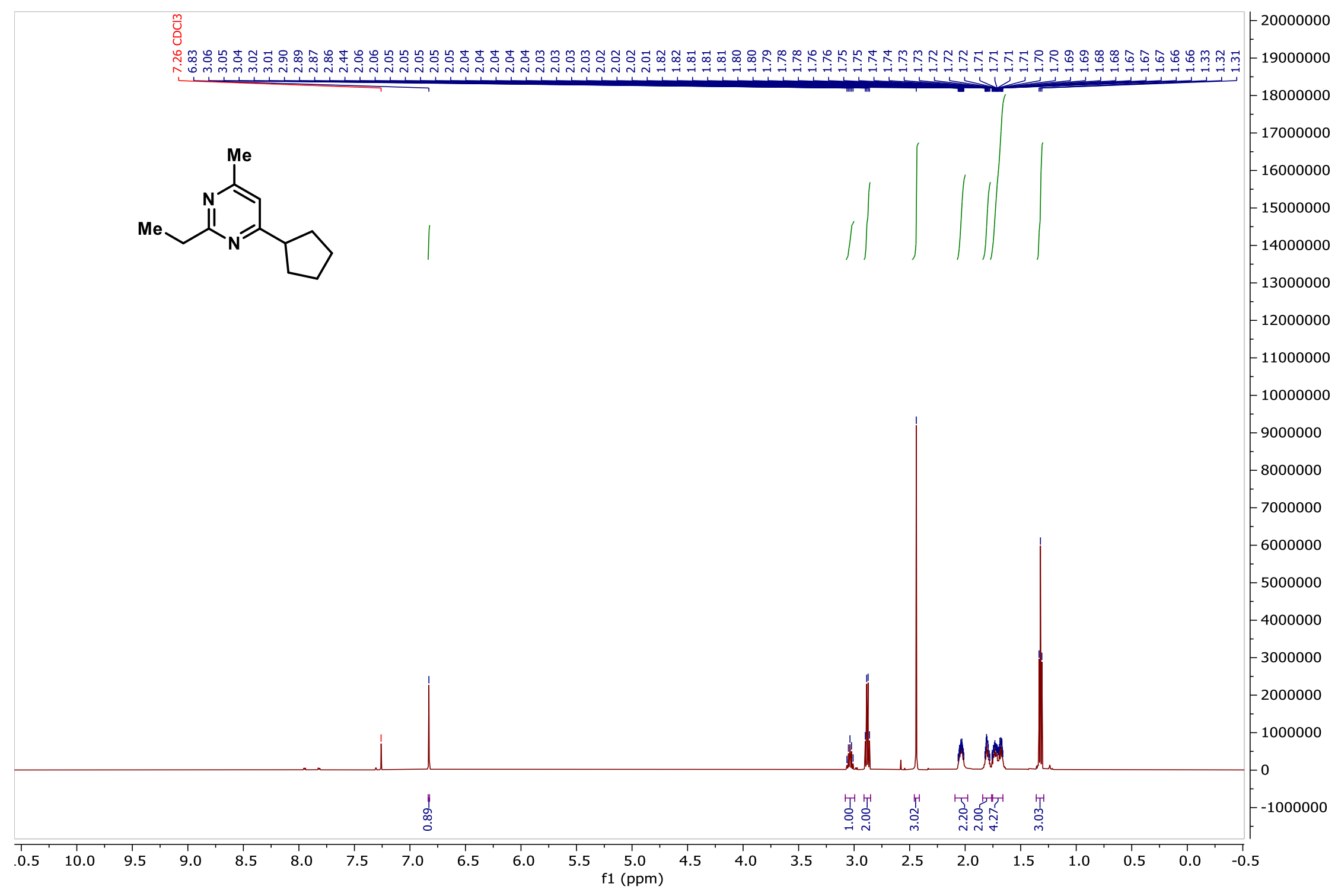


Compound $31,{ }^{13} \mathrm{C}\left\{{ }^{1} \mathrm{H}\right\}$ NMR $\left(\mathrm{CDCl}_{3}, 151 \mathrm{MHz}\right)$

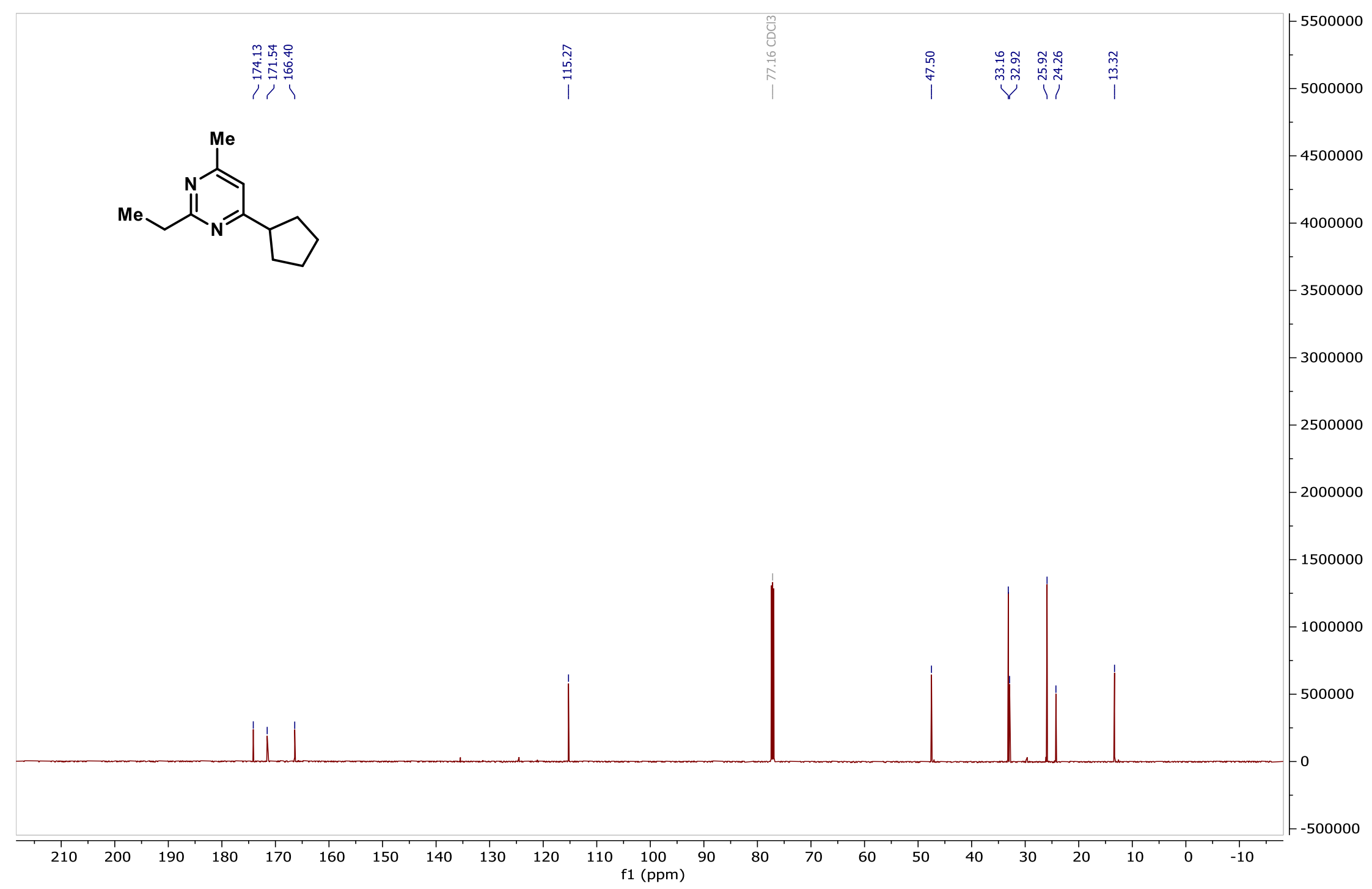


Compound $32,{ }^{1} \mathrm{H}$ NMR $\left(\mathrm{CDCl}_{3}, 600 \mathrm{MHz}\right)$

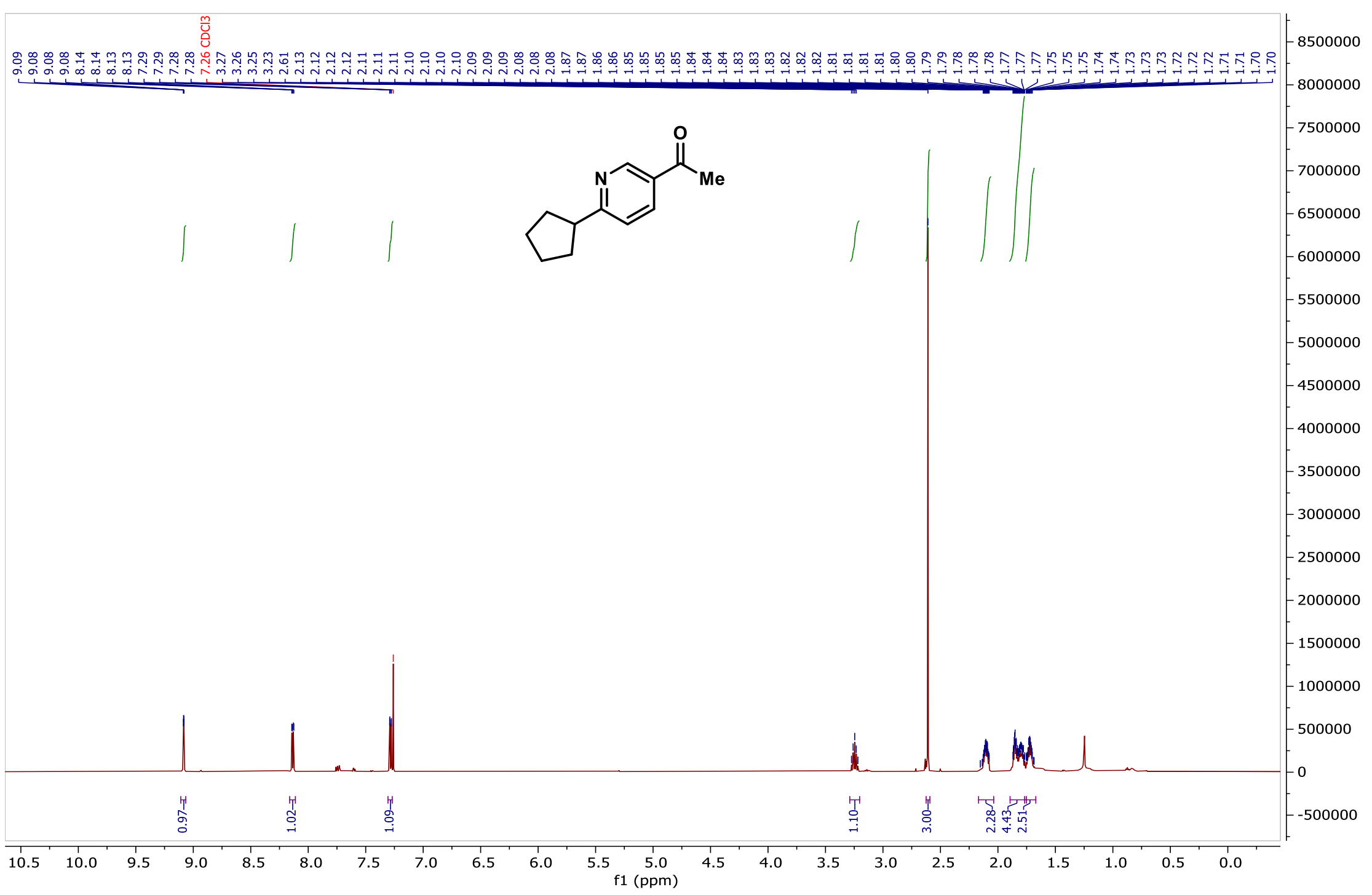


Compound $32,{ }^{13} \mathrm{C}\left\{{ }^{1} \mathrm{H}\right\}$ NMR $\left(\mathrm{CDCl}_{3}, 151 \mathrm{MHz}\right)$

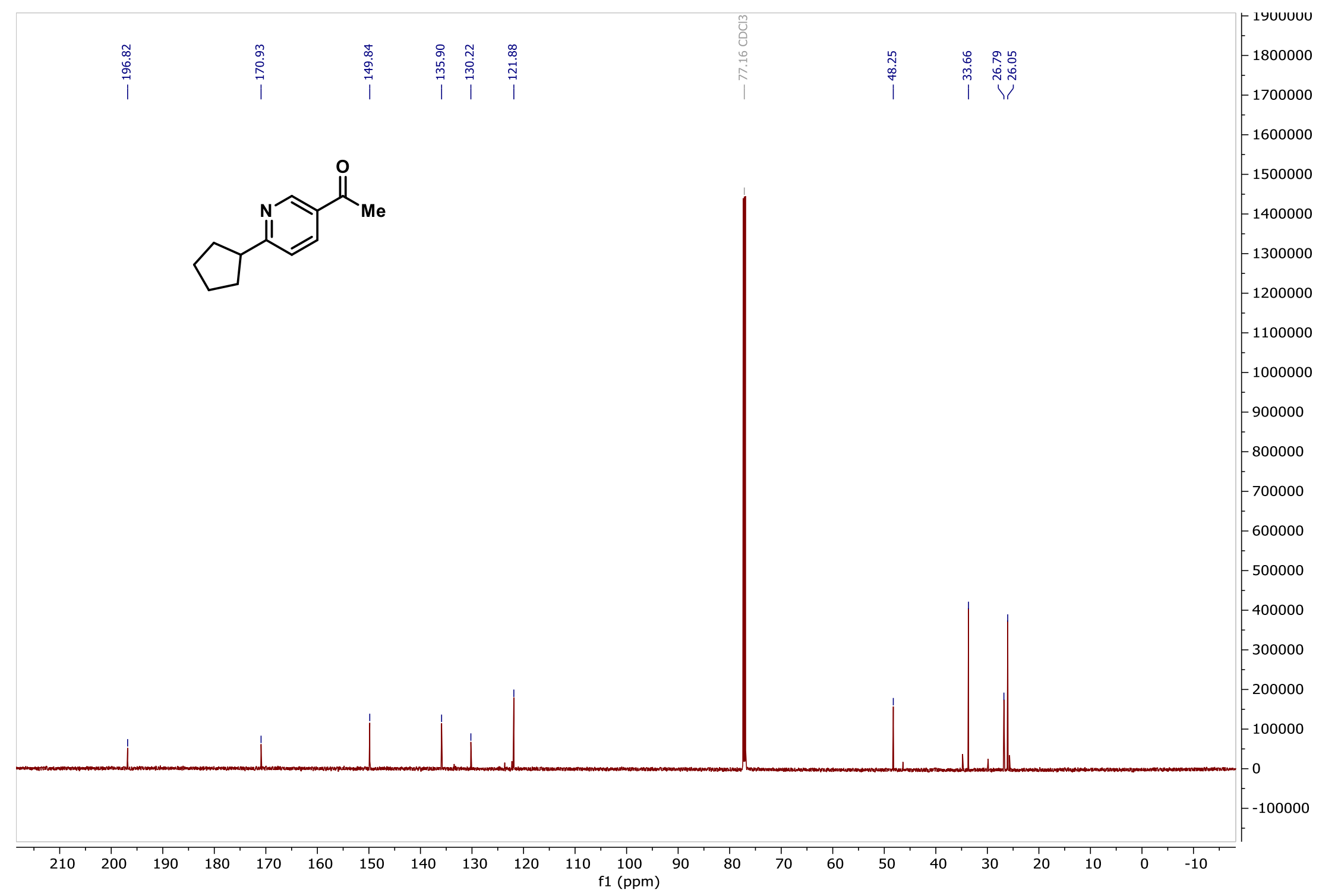


Compound $33,{ }^{1} \mathrm{H}$ NMR $\left(\mathrm{CDCl}_{3}, 600 \mathrm{MHz}\right)$

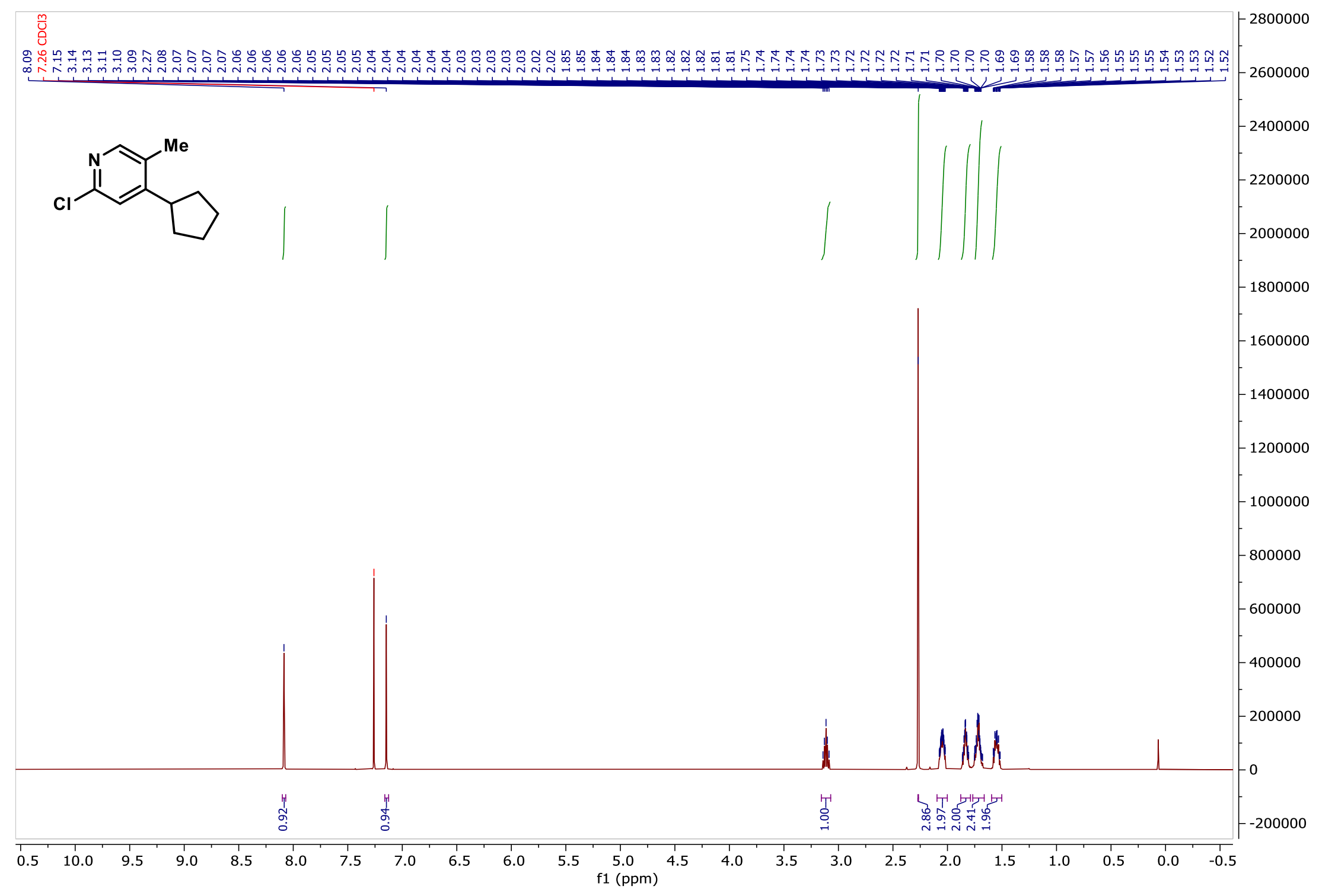


Compound $33,{ }^{13} \mathrm{C}\left\{{ }^{1} \mathrm{H}\right\}$ NMR $\left(\mathrm{CDCl}_{3}, 151 \mathrm{MHz}\right)$

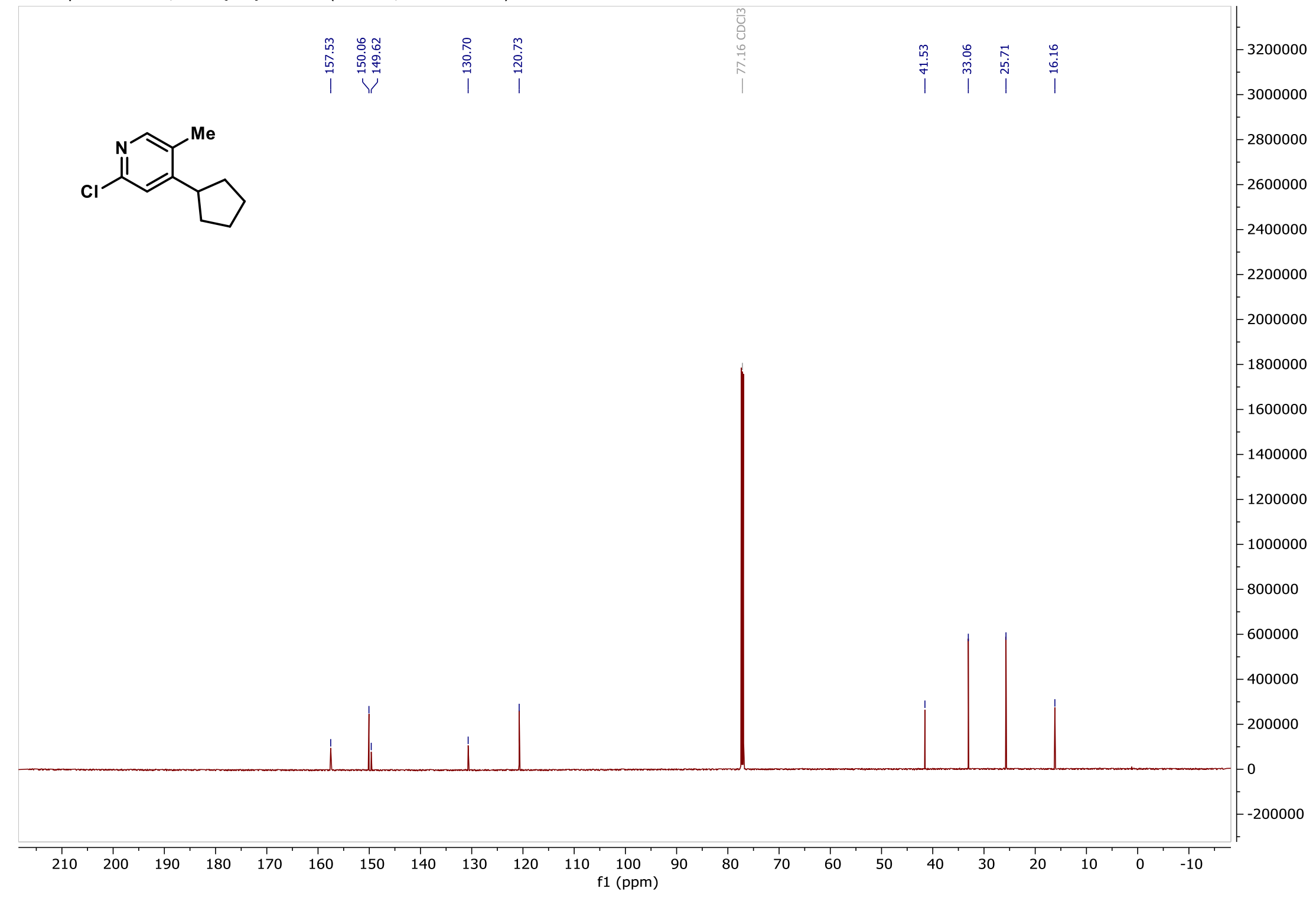


Compound 34, ${ }^{1} \mathrm{H}$ NMR (CDCl $\left.3,600 \mathrm{MHz}\right)$

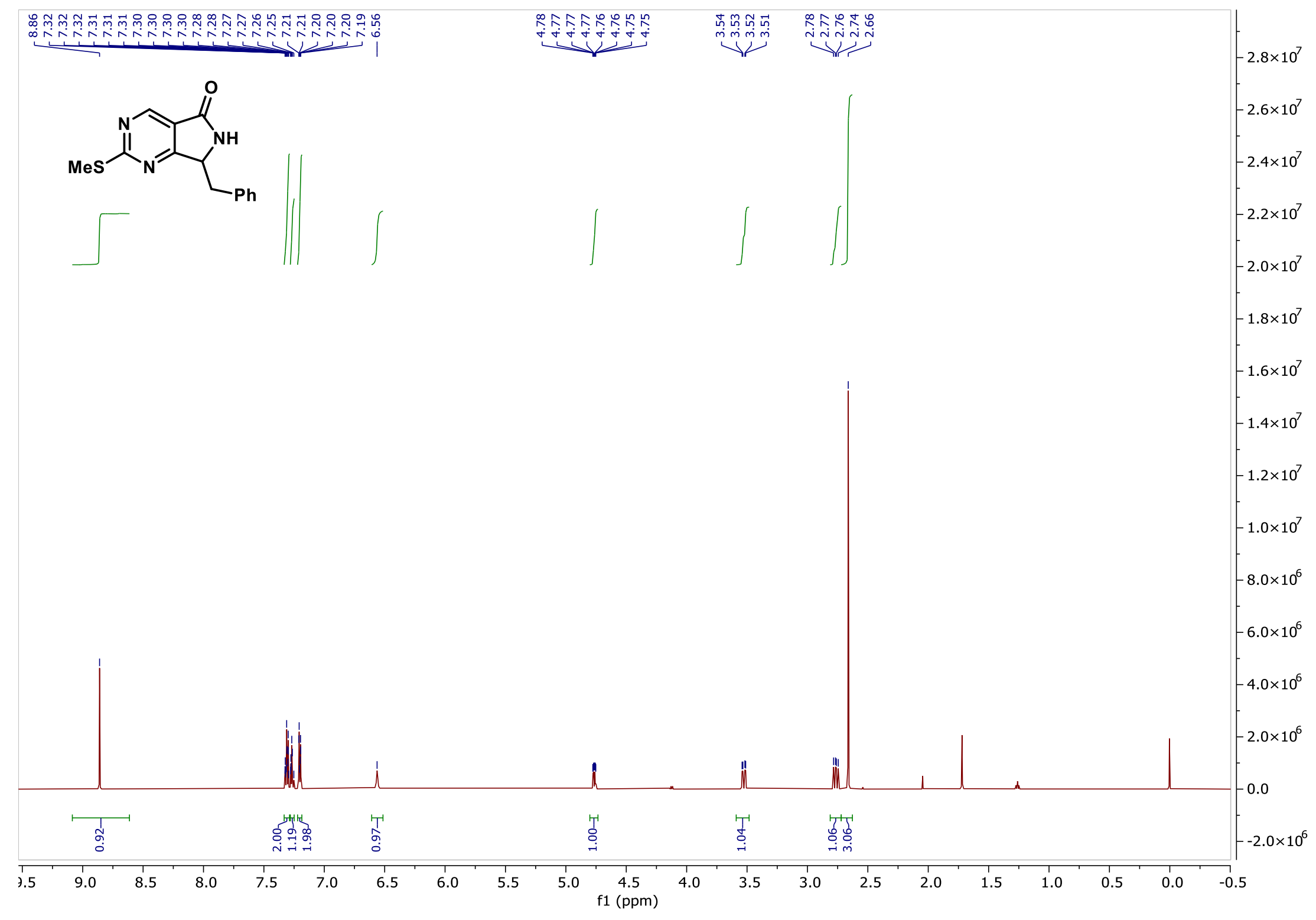


Compound $34,{ }^{13} \mathrm{C}\left\{{ }^{1} \mathrm{H}\right\}$ NMR $\left(\mathrm{CDCl}_{3}, 151 \mathrm{MHz}\right)$

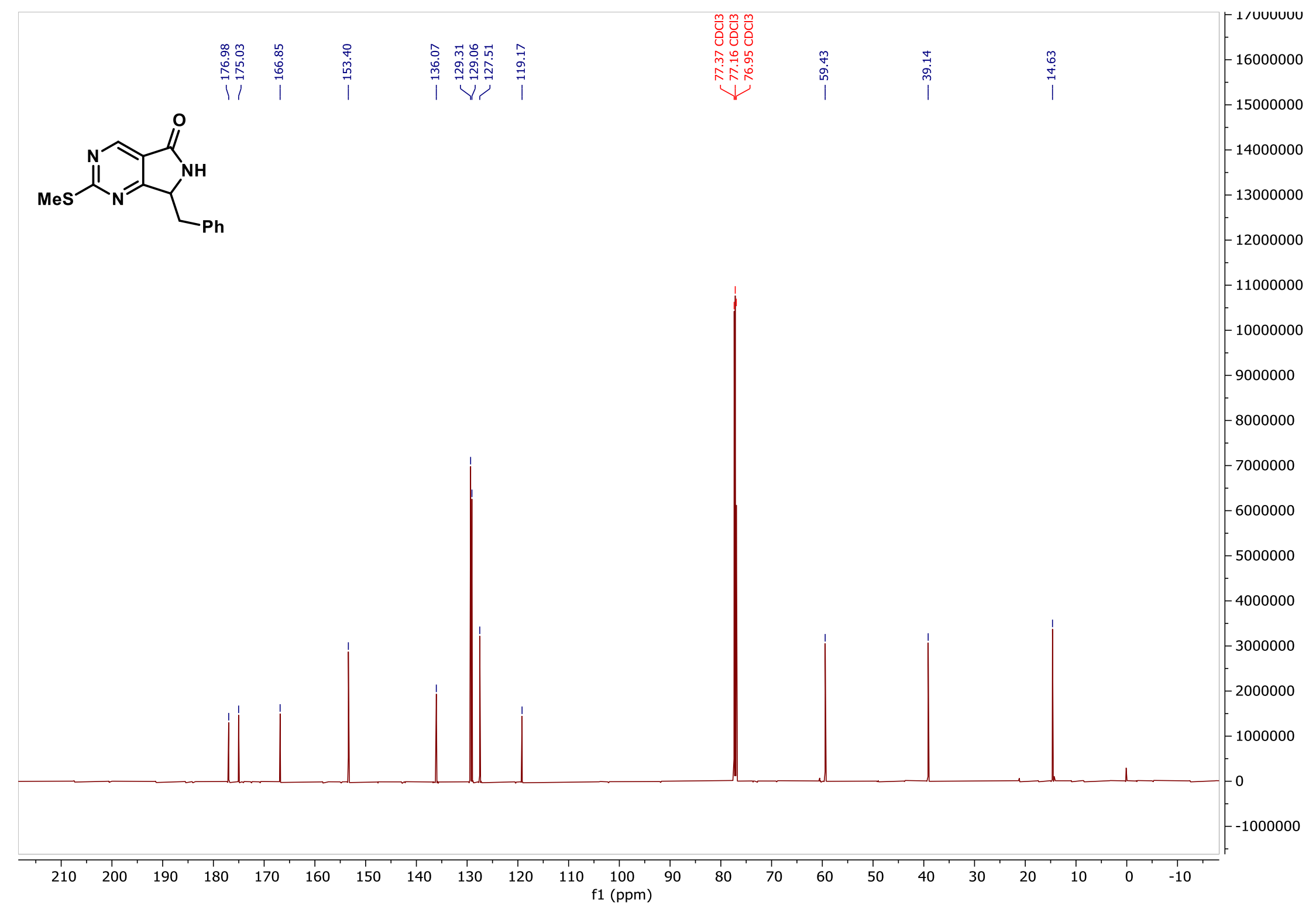


Compound $35,{ }^{1} \mathrm{H} \mathrm{NMR}\left(\mathrm{CDCl}_{3}, 500 \mathrm{MHz}\right)$

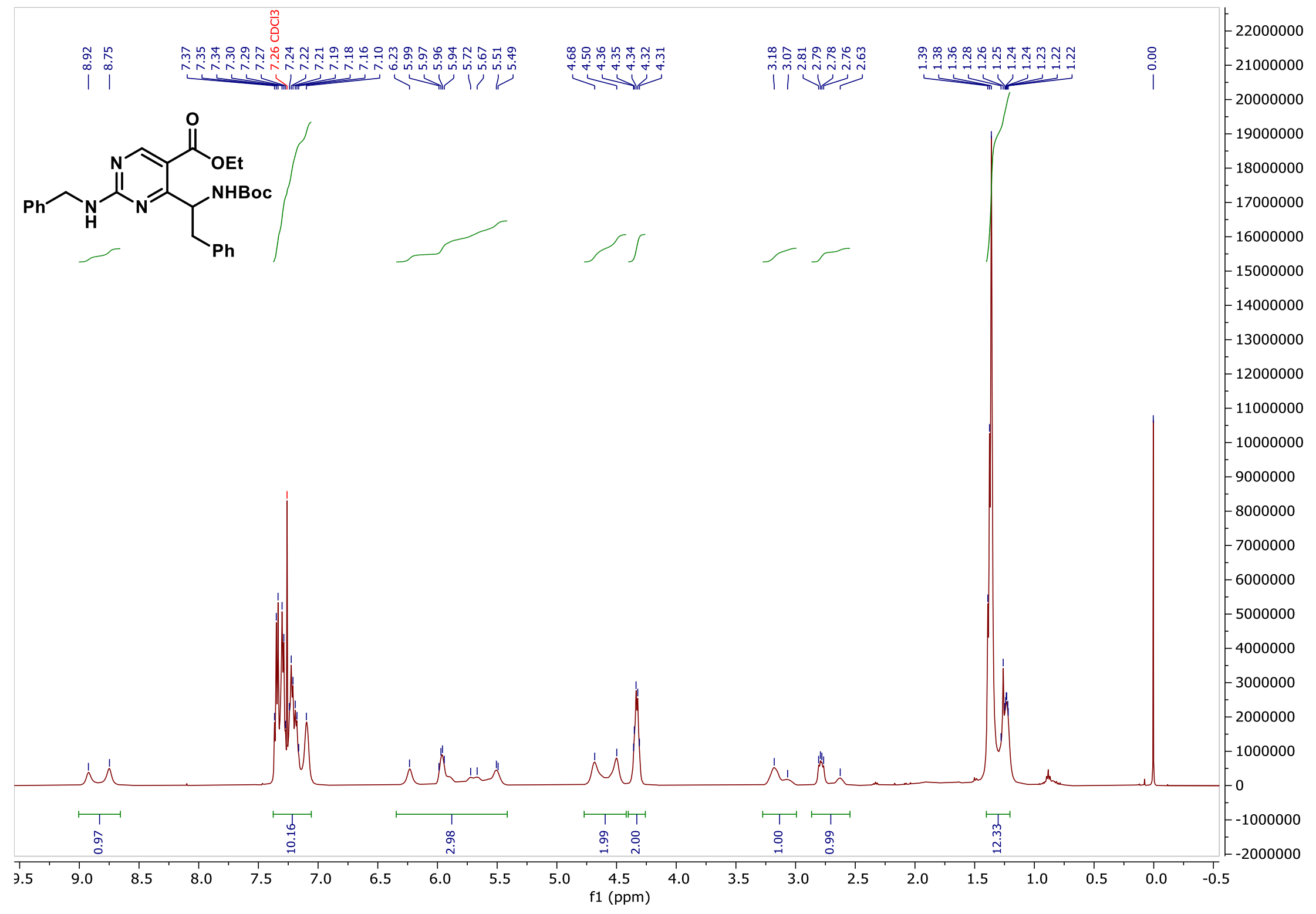


Compound $35,{ }^{13} \mathrm{C}\left\{{ }^{1} \mathrm{H}\right\}$ NMR $\left(\mathrm{CDCl}_{3}, 126 \mathrm{MHz}\right)$

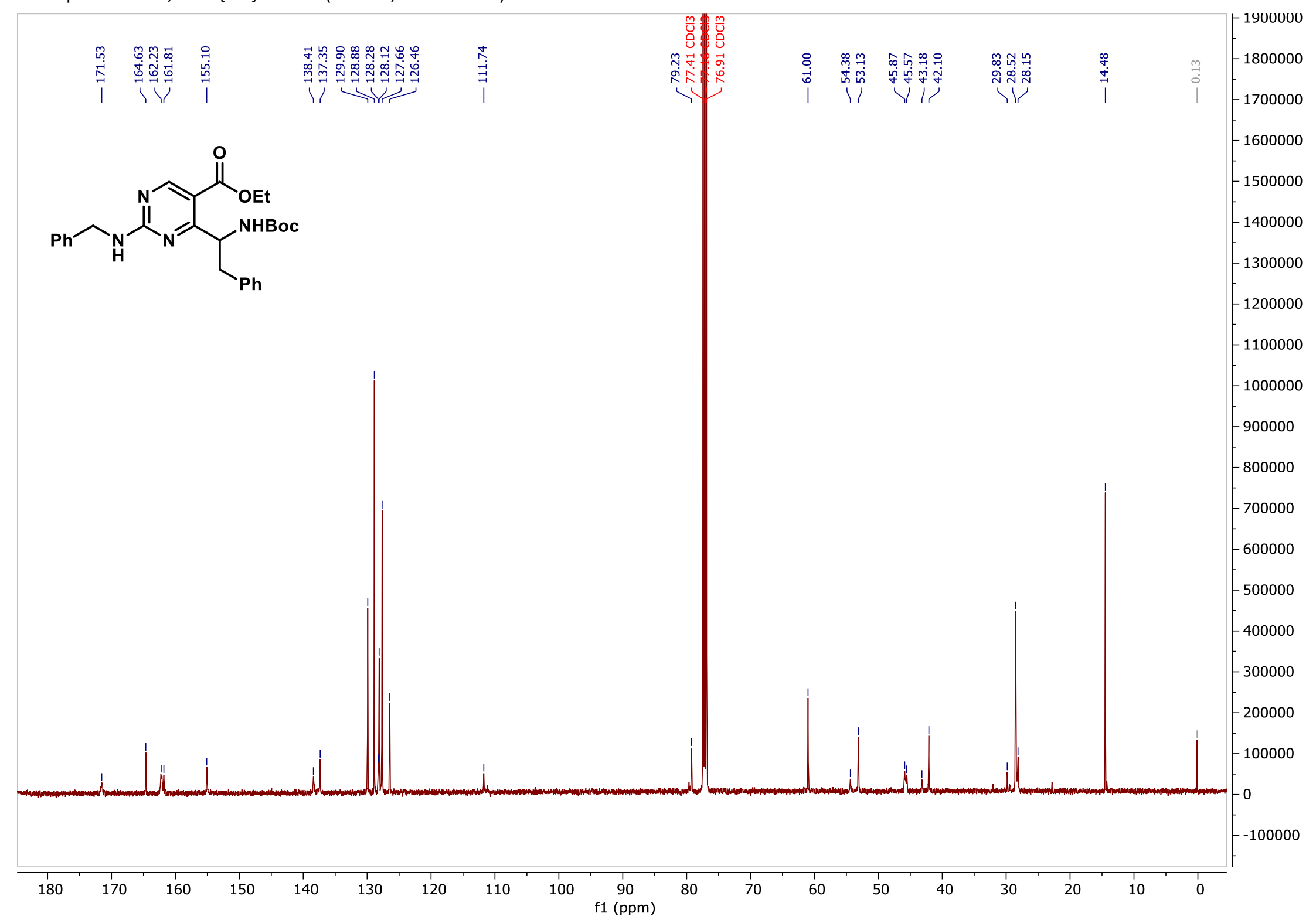

